\title{
Assessment of ORIGEN Reactor Library Development for Pebble-Bed Reactors Based on the PBMR-400 Benchmark
}

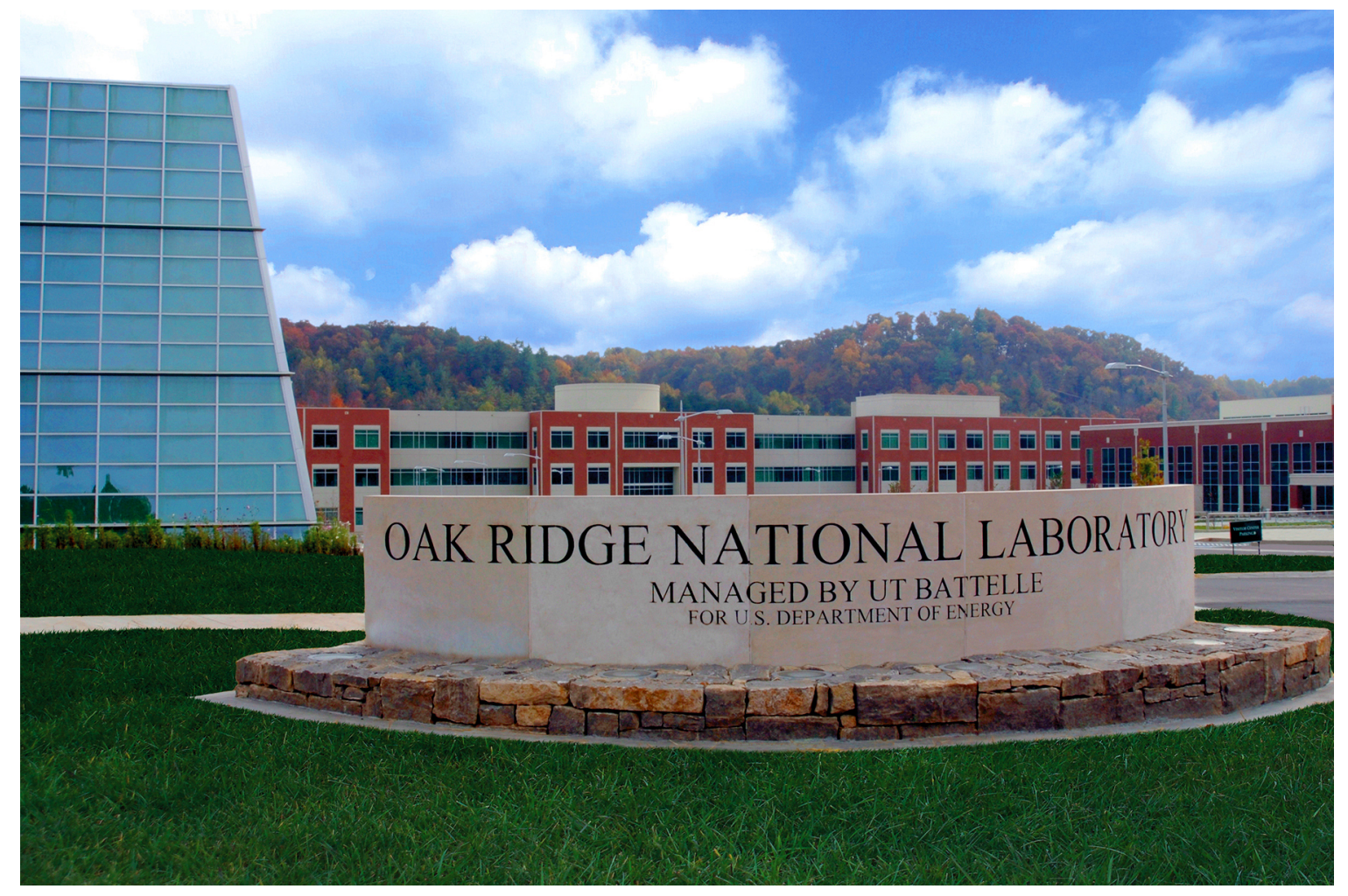

Steven E. Skutnik

William A. Wieselquist

Approved for public release.

Distribution is unlimited.

July 2021

OAK RIDGE 


\section{DOCUMENT AVAILABILITY}

Reports produced after January 1, 1996, are generally available free via US Department of Energy (DOE) SciTech Connect.

Website osti.gov

Reports produced before January 1, 1996, may be purchased by members of the public from the following source:

National Technical Information Service

5285 Port Royal Road

Springfield, VA 22161

Telephone 703-605-6000 (1-800-553-6847)

TDD 703-487-4639

Fax 703-605-6900

E-mail info@ntis.gov

Website classic.ntis.gov

Reports are available to DOE employees, DOE contractors, Energy Technology Data Exchange representatives, and International Nuclear Information System representatives from the following source:

Office of Scientific and Technical Information

PO Box 62

Oak Ridge, TN 37831

Telephone 865-576-8401

Fax 865-576-5728

E-mail reports@osti.gov

Website osti.gov/contact

This report was prepared as an account of work sponsored by an agency of the United States Government. Neither the United States Government nor any agency thereof, nor any of their employees, makes any warranty, express or implied, or assumes any legal liability or responsibility for the accuracy, completeness, or usefulness of any information, apparatus, product, or process disclosed, or represents that its use would not infringe privately owned rights. Reference herein to any specific commercial product, process, or service by trade name, trademark, manufacturer, or otherwise, does not necessarily constitute or imply its endorsement, recommendation, or favoring by the United States Government or any agency thereof. The views and opinions of authors expressed herein do not necessarily state or reflect those of the United States Government or any agency thereof. 
Nuclear Energy \& Fuel Cycle Division

\section{ASSESSMENT OF ORIGEN REACTOR LIBRARY DEVELOPMENT FOR PEBBLE-BED REACTORS BASED ON THE PBMR-400 BENCHMARK}

Steven E. Skutnik

William A. Wieselquist

Date Published: July 2021

Prepared by

OAK RIDGE NATIONAL LABORATORY

Oak Ridge, TN 37831-6283

managed by

UT-Battelle, LLC

for the

US DEPARTMENT OF ENERGY

under contract DE-AC05-00OR22725 


\section{CONTENTS}

LIST OF FIGURES $\ldots \ldots \ldots \ldots \ldots \ldots \ldots \ldots \ldots \ldots \ldots$

LIST OF TABLES $\ldots \ldots \ldots \ldots \ldots \ldots \ldots \ldots \ldots \ldots$ viii

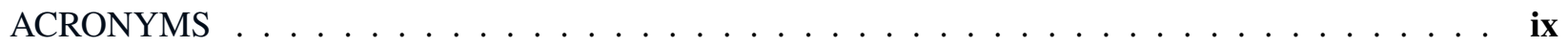

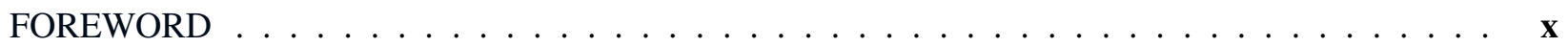

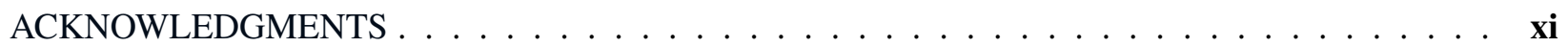

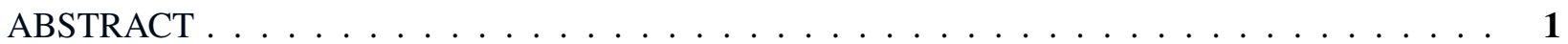

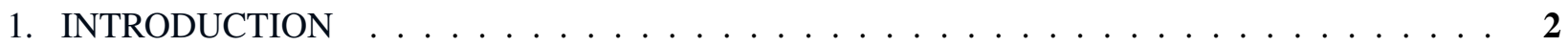

1.1 PRIOR ANALYSES USING SCALE OF PEBBLE-BED TYPE SYSTEMS . . . . . . . . 3

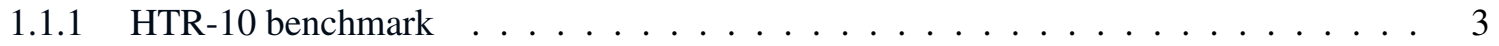

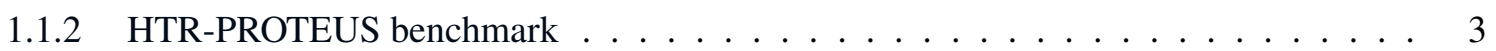

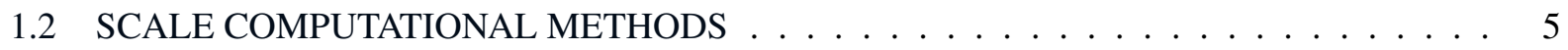

1.2.1 Doubly heterogeneous fuel treatment methods in SCALE . . . . . . . . . . . . 5

1.2.2 Shift capabilities specific to TRISO fuel particles . . . . . . . . . . . . . . 5

1.3 PRIOR APPROACHES TO PEBBLE-BED REACTOR PHYSICS ANALYSIS $\ldots \ldots$

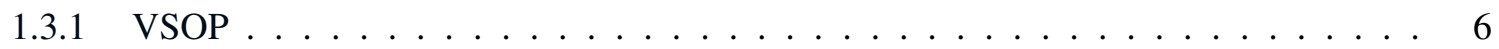

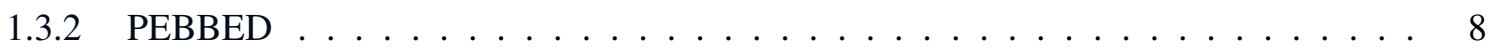

1.4 OVERVIEW OF THE PBMR-400 BENCHMARK PROBLEM . . . . . . . . . . . . . . . 9

2. PBMR-400 MODEL VERIFICATION . . . . . . . . . . . . . . . . 11

2.1 SINGLE, REFLECTED PEBBLE MODEL $\ldots \ldots \ldots \ldots \ldots \ldots \ldots$

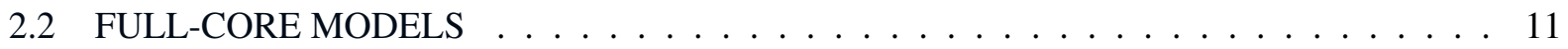

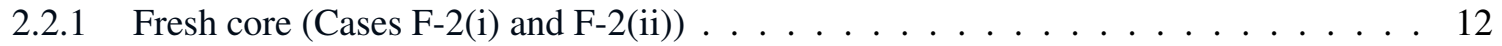

2.2.2 Equilibrium-composition core (Cases E-1(i) and E-1(ii)) . . . . . . . . . . . 13

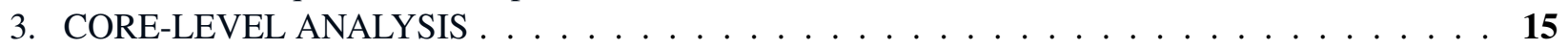

3.1 SPATIAL DISTRIBUTION OF THE NEUTRON FLUX AND CORE POWER . . . . . . 15

3.1.1 Total flux and fission source gradients over the core . . . . . . . . . . . . . . 15

3.1.2 Spatial differences in neutron spectrum characteristics . . . . . . . . . . . 16

3.2 CORE PHYSICS PARAMETERS . . . . . . . . . . . . . . . . . . . . . . . . . . . . 19

3.2.1 Temperature coefficient of reactivity . . . . . . . . . . . . . . . . . . 19

3.2.2 Energy of the average lethargy causing fission . . . . . . . . . . . . . . . . . 19

4. DEVELOPMENT OF ONE-GROUP ORIGEN CROSS-SECTION LIBRARIES . . . . . . . . . 21

4.1 CONSISTENCY OF MODEL SCOPE FOR MULTI-GROUP MONTE CARLO CALCU-

LATIONS FOR LIBRARY GENERATION ．. . . . . . . . . . . . . . . . . . . 21

4.1.1 Burnup-dependent effects on the neutron spectrum . . . . . . . . . . . . 22

4.1.2 Differences in the neutron spectra arising from radial position . . . . . . . . . . 25

4.1 .3 Comparison to historic 1 -D approaches . . . . . . . . . . . . . . . 28

4.2 EFFECTS OF POWER HISTORY ON ONE-GROUP CROSS-SECTIONS . . . . . . . . . 28

4.3 TEMPERATURE EFFECTS ON ONE-GROUP CROSS-SECTION LIBRARIES . . . . . . 29

4.4 EFFECT OF MODEL SCOPE ON CALCULATED ONE-GROUP CROSS-SECTIONS _ . 33

4.5 COMPARISON OF HTGR AND LWR LIBRARY CHARACTERISTICS . . . . . . . . . 35

4.5 .1 BWR assemblies . . . . . . . . . . . . . . . . . . 35

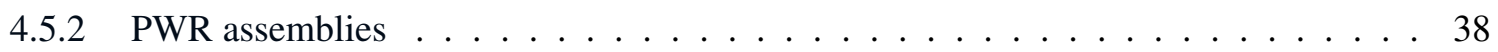

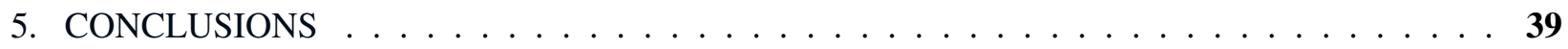

5.1 DEPLETION MODELING FROM A "PEBBLE-LEVEL" APPROACH $\ldots \ldots$. . . . . . . 40

5.2 RECOMMENDATIONS FOR ORIGEN LIBRARY DEVELOPMENT . . . . . . . . . . . . 41

5.3 RECOMMENDED DEVELOPMENT PRIORITIES FOR ORIGAMI . . . . . . . . . . . 41

5.3 .1 Enhancements to library interpolation . . . . . . . . . . . . . . . 41 


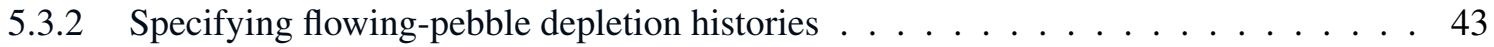

5.4 ADDITIONAL ANALYTICAL CAPABILITIES TO BE DEVELOPED . . . . . . . . . . 44

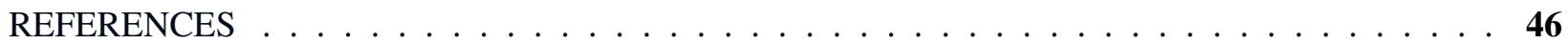

A. DEVELOPMENT OF A COMPACT SPHERICAL PACKING ALGORITHM . . . . . . . . . . . A-1

B. NEUTRONIC ANALYSIS OF THE FRESH CORE . . . . . . . . . . . . . . . . . . . B-1 


\section{LIST OF FIGURES}

1 VSOP calculation flow for Mehrfachdurchlauf (MEDUL) (multi-pass) cores [15] $\ldots \ldots$

2 SCALE 6.3 single-pebble reflected model of a fresh PBMR-400 pebble, representing an infinite array of pebbles. . . . . . . . . . . . . . . . . . . . 11

3 SCALE 6.3 model for the PBMR-400 fresh core, consisting of $1 / 3$ fuel pebbles (green) and $2 / 3$ graphite "dummy" pebbles, hexagonal close-packed (HCP) (dodecahedral) arrangement; (left) axial cutaway view, (right) axial cut plane views at $z=-100 \mathrm{~cm}$, where $z=0$ represents the top of the active fuel region. . . . . . . . . . . . . . . 13

4 SCALE 6.3 model for the PBMR-400 equilibrium-composition core, dodecahedral lattice / HCP arrangement; (left) axial cutaway view, (right) axial cut plane view at $z=100 \mathrm{~cm} . ~ \ldots . ~ 14$

5 PBMR-400 flux distribution for the equilibrium-composition core; (left) total flux, (center) fast flux $(E>1.86 \mathrm{eV})$, (right) thermal flux $(E<1.86 \mathrm{eV})$. Red indicates regions of highest

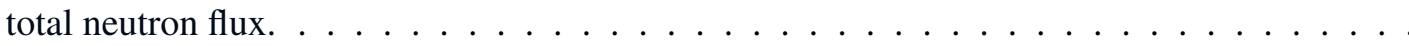

6 Lethargy-weighted neutron energy spectrum by radial zone (numbered innermost to outermost) for equilibrium-composition core, evaluated at each of the 22 axial material zones. Solid lines represent the average group flux within the radial zone (over all axial zones), with shaded region indicating the standard deviation of the intra-zone flux distribution. . . .

$7 \quad$ PBMR-400 fast flux $(E>1.86 \mathrm{eV})$ distribution for equilibrium-composition core at $1200 \mathrm{~K}$ isotherm; (left) Radial fast flux by axial elevation $(z=0 \mathrm{~cm}$ corresponds to the top of the active fuel zone); (right) axial fast flux profile by radial zone. . . . . . . . . . . . . . .

8 PBMR-400 thermal flux $(E<1.86 \mathrm{eV})$ distribution for equilibrium-composition core at $1200 \mathrm{~K}$ isotherm; (left) Radial thermal flux by axial elevation $(z=0 \mathrm{~cm}$ corresponds to the top of the active fuel zone); (right) axial thermal flux profile by radial zone. . . . . . . . . .

9 Fission power factor for the PBMR-400 equilibrium-composition core by axial location and radial region. Note that $z=0 \mathrm{~cm}$ indicates the top of the active fuel zone. . . . . . . . 18

10 Spectral ratio (fast : thermal flux) for the equilibrium-composition core, along the (left) axial dimension, (right) radial dimension; the thermal-fast boundary is defined at $E=1.86 \mathrm{eV}$. Note that $z=0 \mathrm{~cm}$ indicates the top of the active fuel zone. . . . . . . . . . . . . 18

11 Fitted system reactivity as a function of temperature for isothermal PBMR-400 systems. (Empty circles represent VSOP values from the benchmark problem used for comparison [1]) 20

12 SCALE 6.3 "reflected plane" model, representing an axially-reflected "slice" of the PBMR system at the core axial mid-plane. Rendering is clipped to illustrate detail; the full radial plane is explicitly modeled. . . . . . . . . . . . . . . . .

13 The 252-group collapsing spectrum as a function of burnup for the single, infinitely reflected pebble and a reflected axial plane (core mid-plane), starting from a fresh pebble / plane configuration. The reflected axial plane model includes $2 / 3$ graphite pebbles, significantly influencing the spectral shape. Note that both models show a burnup-dependent spectral shift, but the effect is more pronounced for the plane. . . . . . . . . . . . . .

14 The 252-group collapsing spectrum as a function of radial location for the reflected plane model (equilibrium fuel composition, $1200 \mathrm{~K}$ system temperature), at the top, middle, and bottom axial regions (locations 1,11 and 22 , respectively) . . . . . . . . . . .

15 The 252-group collapsing spectrum as a function of radial location for the reflected plane model, full-core model, and the infinitely-reflected pebble, based on equilibrium fuel compositions and a system isotherm of $1200 \mathrm{~K}$. 
16 Collapsed one-group removal cross-sections for major actinides for the PBMR-400 equilibriumcomposition core for varying axial positions in the (left) reflected plane model, for three separate axial regions (top, middle, bottom) and (right) full-core model (22 axial regions); all evaluations conducted at $1200 \mathrm{~K}$ isotherm. . . . . . . . . . . . . . . . . 25

17 Collapsed one-group removal cross-sections for major actinides for the the infinitely reflected pebble (equilibrium-composition core) as a function of temperature and burnup. . . . 26

18 252-group collapsing spectrum as a function of radial location for a single (fresh) pebble depleted within a static radial plane of pebbles at equilibrium composition (referred to as the "marinade" model), with the depleted pebble at approximately $90 \frac{\mathrm{GWd}}{\mathrm{MTIHM}}$ burnup and a

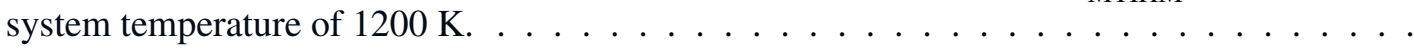

19 One-group removal cross-section for major actinides as a function of radial location and burnup for a single (fresh) pebble depleted to approximately $90 \frac{\mathrm{GWd}}{\mathrm{MTIHM}}$ within a static radial plane of pebbles at equilibrium composition with a system temperature of $1200 \mathrm{~K}$. . . . . 28

20 Comparative power histories used representing one transit of a pebble through the core . . . 29

21 Effective one-group removal cross-sections for the single reflected (fresh) pebble, irradiated at uniform and non-uniform power histories over one core transit (approximately 17.6 $\frac{\mathrm{GWd}}{\mathrm{MTIHM}}$ ) for (left) $300 \mathrm{~K}$ and (right) $1200 \mathrm{~K}$ system isotherm. . . . . . . . . . . . . . .

22 The 252-group collapsing spectrum as a function of temperature (system isotherm) for the fresh core, reflected plane model, with corresponding radiative capture cross-sections for major actinidess overlaid for reference. Note the shift of the thermal neutron distribution onto the ${ }^{239} \mathrm{Pu}$ giant resonance with increasing temperature. . . . . . . . . . . . . . . .

23 The 252-group collapsing spectrum as a function of temperature (system isotherm) for the equilibrium-composition core, reflected plane model, with corresponding radiative capture cross-sections for major actinidess overlaid for reference. Variations in the flux shape by radial zones are represented as shaded areas. . . . . . . . . . . . . . .

24 One-group removal cross-section for major actinides as a function of temperature $(\mathrm{K})$ and radial location for the equilibrium-composition core (full core model). Note the divergence in the removal cross-sections for fissile plutonium isotopes ${ }^{239} \mathrm{Pu}$ and ${ }^{241} \mathrm{Pu}$ between the outer radial zones and the interior zones with increasing temperature. . . . . . . . . . . .

25 (Left) Absolute and (right) relative difference in calculated one-group removal cross-sections for major actinides for the reflected plane and infinite pebble models. . . . . . . . . . . .
Energy of the average lethargy causing fission for various BWR assembly types under a range of moderator void fractions, fuel and moderator temperatures, and presence of control rods, with data from [24]. The calculated (overlapping) ranges of EALF values for the PBMR fresh and equilibrium-composition cores (calculated solely as a function of system isotherm temperature) are presented for comparison. . . . . . . . . . . . . 36

27 Energy of the average lethargy causing fission for a GE14 BWR assembly, broken out by lattice zone configurations per conventions defined in [25] (e.g., dominant lattice zone (DOM), power-shaping zone (PSZ)), using data from [24]. The calculated (overlapping) ranges of EALF values for the PBMR fresh and equilibrium-composition cores (calculated solely as a function of system isotherm temperature) are presented for comparison. . . . . . . . . . 36

28 Comparative lethargy-weighted multi-group energy spectra for the GE14 fresh assembly under CZP (0\% void) and HFP (90\% void) (representing relative lethargy extrema for the BWR cases considered), alongside the PBMR equilibrium-composition cores at 300 and $1200 \mathrm{~K}$. Shaded regions represent variations over different (non-Gd) fuel regions within the

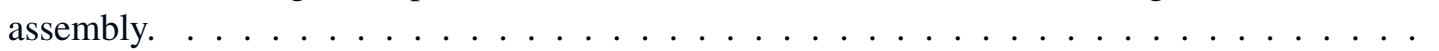


29 Energy of the average lethargy causing fission for various PWR assembly types under a range of initial fuel enrichments, fuel and moderator temperatures, soluble boron concentrations, and presence of control rods, with data from [24]. The calculated ranges of EALF values for the PBMR fresh and equilibrium-composition cores (calculated solely as a function of system isotherm temperature) are presented for comparison. . . . . . . . . . . . . 38

30 Proposed series of "map overlays" for interpolation of libraries with arbitrary dimensions in ORIGAMI; the combination of the maps produces a unique tuple for each depletion location ("pebble"), resulting in the permutations shown in Table $11 . \ldots . . . . . . . . .442$

31 Sample implementation of an ORIGAMI interface for flowing-pebble high-temperature gas reactor $(\mathrm{HTGR})$ systems. . . . . . . . . . . . . . . . . . . . . . . 44

32 PBMR-400 flux distribution for the fresh (initial) core; (left) fast neutron group $(E>1.86$ $\mathrm{eV})$, (right) thermal group $(E \leq 1.86 \mathrm{eV}) \ldots \ldots \ldots \ldots \ldots \ldots$. . . . . . . . . . . . . . .

33 Spectral ratio (fast : thermal flux) for the fresh core, along the (left) axial dimension, (right) radial dimension; the thermal-fast boundary is defined at $E=1.86 \mathrm{eV} \ldots \ldots \ldots$. . . . . B-3 


\section{LIST OF TABLES}

1 Deterministic pebble configurations modeled with SCALE for the HTR-PROTEUS benchmarks (adapted from [5]) . . . . . . . . . . . . . . . . . . . . . . 4

2 Key physical dimensions of the PBMR-400 reactor [2], [3] . . . . . . . . . . . . . 10

3 Selected PBMR-400 neutronic benchmark problems, from [2] . . . . . . . . . . . . 10

4 Comparison of fresh core PBMR-400 benchmark model to reported $\mathrm{k}_{\mathrm{eff}}$ values from Tables 4.27 and 4.28 of [2], corresponding to cases F-2(i) (cold zero power (CZP)), and F-2(ii) (hot zero power $(\mathrm{HZP})) \ldots \ldots \ldots \ldots \ldots \ldots \ldots \ldots \ldots$

5 Comparison of E-1(i) and E-1(ii) equilibrium-composition core PBMR-400 benchmark model with reported $k_{\text {eff }}$ values from Tables 4.27 and 4.28 of [2] . . . . . . . . . . . . . . . . 14

6 Estimated temperature coefficient of reactivity for PBMR-400 fresh and equilibrium-composition cores . . . . . . . . . . . . . . . . . . . . . . . 19

7 Energy of the average lethargy causing fission $(\mathrm{eV})$ as a function of core temperature. . . . 20

8 Pebble dimensions and modeling assumptions used for characterization of non-uniform power history on discharge isotopic composition. . . . . . . . . . . . . . . . . . . 29

9 Impact of library-generating model on discharge isotopic inventories for a single-transit depletion cycle, expressed as the relative inventory difference compared with depletion using a one-group library derived from the full-core model. Full core and reflected plane models assume the pebble is at the radially centermost location (farthest from reflectors), core axial

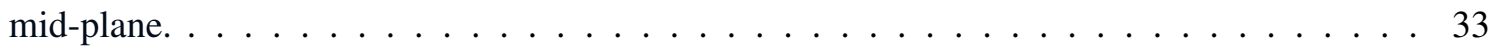

10 Impact of radial library position on isotopic inventories after one pass through the core (a burnup of approximately 17.6 $\frac{\mathrm{GWd}}{\mathrm{MTIHM}}$ ), expressed as the relative mass difference (in percentage) compared with depletion using the respective one-group library corresponding to the centermost radial location (radial position \#3). . . . . . . . . . . . . 34

11 Pebble "permutations" of initial enrichment, radial location, and starting burnup, as given by the overlaid tables shown in Figure $30 \ldots \ldots \ldots \ldots \ldots$ 


\section{ACRONYMS}

$\begin{array}{ll}\text { AVR } & \text { Arbeitsgemeinshaft Versuchsreaktor } \\ \text { BWR } & \text { boiling water reactor } \\ \text { CHPOP } & \text { columnar hexagonal point-on-point } \\ \text { CZP } & \text { cold zero power } \\ \text { DOM } & \text { dominant lattice zone } \\ \text { EALF } & \text { energy of the average lethargy causing fission } \\ \text { FCC } & \text { face-centered cubic } \\ \text { HCP } & \text { hexagonal close-packed } \\ \text { HFP } & \text { hot full power } \\ \text { HTGR } & \text { high-temperature gas reactor } \\ \text { HZP } & \text { hot zero power } \\ \text { INL } & \text { Idaho National Laboratory } \\ \text { IRPhE-Handbook } & \text { International Handbook of Evaluated Reactor Physics Benchmark Experiments } \\ \text { LWR } & \text { light water reactor } \\ \text { MEDUL } & \text { Mehrfachdurchlauf } \\ \text { MOX } & \text { mixed oxide } \\ \text { MWt } & \text { megawatts thermal } \\ \text { NAT } & \text { natural uranium axial blanket region } \\ \text { OTTO } & \text { One Through Then Out } \\ \text { PBMR } & \text { pebble bed modular reactor } \\ \text { PBR } & \text { pebble bed reactor } \\ \text { PCD } & \text { pitch circle diameter } \\ \text { pcm } & \text { percent mille } \\ \text { PLE } & \text { plenum region } \\ \text { PSZ } & \text { power-shaping zone } \\ \text { PWR } & \text { pressurized water reactor } \\ \text { RCP } & \text { random close-packed } \\ \text { RCS } & \text { reactor control system } \\ \text { RPV } & \text { reactor pressure vessel } \\ \text { THTR } & \text { thorium high temperature reactor } \\ \text { TRISO } & \text { vanished lattice zone } \\ \text { UOX } & \\ \text { VAN } & \end{array}$




\section{FOREWORD}

This work establishes the key system parameters to enable rapid fuel inventory generation for pebble-based HTGR systems within SCALE. For light water reactor light water reactor (LWR) assemblies this process is well-established. For example, in the boiling water reactor (BWR), the moderator density and burnup were found to be the key system parameters affecting inventory calculations.

This study uses the PBMR-400 (pebble bed modular reactor) benchmark problem as a basis for quantifying the effects of system parameters on the multi-group energy spectrum and its attendant effects on the effective one-group cross-sections used for depletion calculations. Examples of these effects include the relative pebble location within the core (axially and radially with respect to the graphite reflectors), the system isotherm temperature, and the effective pebble burnup.

Our findings indicate that, unlike in traditional LWR assemblies, the primary drivers of the effective neutron spectrum and one-group cross-sections are the pebble radial distance from the reflector region and the system temperature (chiefly attributable to the temperature-dependent scattering effects in the graphite mod-

erator). Because of the continuously circulating nature of the fuel (including continuous replacement of fully burned pebbles with fresh ones), factors such as fuel burnup (which are typically first-order parameters) exhibit significantly attenuated response compared with factors such as the radial location and system temperature. This finding would imply that the development of ORIGEN reactor libraries for pebble-based HTGR systems will require substantively new approaches for parameter interpolation for rapid depletion calculations, compared with methods presently employed for LWR libraries. 


\section{ACKNOWLEDGMENTS}

This work was performed under the support of NRC Contract IAA 31310019N0012; the authors wish to gratefully acknowledge the support of the Nuclear Regulatory Commission for this work, and in particular program manager Don Algama.

The authors likewise wish to acknowledge the contributions of numerous technical staff who assisted in the development of this work by providing thoughtful feedback and ideas throughout. Specifically, the authors wish to express sincere appreciation to Rike Bostelmann, Ugur Mertyurek, and Germina Ilas, each of whom provided helpful feedback and discussion throughout the course of this project. A particular thanks goes to Ugur Mertyurek for providing access to the results of previous reactor physics calculations for pressurized water reactor (PWR) and BWR assemblies in order to facilitate direct comparisons with the physics characteristics of the pebble bed modular reactor (PBMR) system. 


\begin{abstract}
This report provides an evaluation of present SCALE capabilities for modeling depletion of pebble-bed reactor systems, using the PBMR-400 benchmark as a test case. A specific aim of this work is to understand the system characteristics required to generate production-quality reactor data libraries for rapid depletion calculations with ORIGEN. This report includes a discussion of present SCALE capabilities for modeling doubly heterogeneous fuels, prior SCALE work modeling pebble bed-type reactors, and a detailed neutronic analysis of the PBMR-400 core for both fresh and equilibrium-composition core conditions.

Validation of the PBMR-400 reactor model is provided through comparisons of the system $\mathrm{k}_{\mathrm{eff}}$ at different temperatures for fresh and equilibrium full-core models evaluated using the CSAS for eigenvalue analysis and TRITON for depletion analysis. For the fresh core, absolute differences in eigenvalues of 97-570 percent mille ( $\mathrm{pcm}$ ) were observed between SCALE/CSAS calculations in comparison to the VSOP reference calculation, indicating relatively good agreement with the benchmark. For the equilibrium-composition core, the observed differences ranged from +728 to $-737 \mathrm{pcm}$, indicating general overall agreement with the VSOP benchmark. Notable factors influencing the $\mathrm{k}_{\mathrm{eff}}$ agreement included representation of the pebble distribution within the core, particularly near the reactor boundaries. Because of the nature of random close packing of hard spheres, regularly spaced lattice representations (such as a face-centered cubic (FCC) dodecahedral lattice) exhibited challenges in representing local packing fractions near the core boundary compared with what would be observed in a random close-packed (RCP) arrangement, despite the fact that these representations were able to reproduce the prescribed global average pebble packing fraction (61\%). Models that made use of a non-physical approximation of truncated or "clipped" pebbles at the core boundary showed the best agreement to the system benchmark, although based upon prior experience, the introduction of a random close-packing capability would be expected to show superior agreement.
\end{abstract}

Based upon an analysis of the spatial and time-dependent characteristics of the neutron spectrum of the equilibrium-composition core, the primary dimensions defining the response space for the development of one-group reactor data libraries (i.e., the parameters used for interpolation of reactor data libraries to problem-specific conditions) were identified. For the PBMR-400 core, these first-order parameters of interest included the fuel initial enrichment, the system temperature, and the pebble radial location relative to the graphite reflector. However, owing to the continuously circulating nature of the fuel (including continuous replacement of pebbles at the terminal discharge burnup by fresh pebbles), for equilibrium core conditions, burnup exhibits a relatively attenuated effect on the neutron energy spectrum (and thus the one-group crosssections); its effect is far more prominent for the case of the fresh core. Moreover, with the exception of axial regions adjacent to inserted control rods, the shape of the neutron energy spectrum (in terms of the thermal to fast neutron flux ratio) is relatively invariant axially. This would potentially indicate that explicit axial location tracking of the pebbles may not be necessary for performing accurate depletion analyses. Rather, tracking the radial positions of the pebbles relative to the reflector region, combined with the effective temperature of the reflector medium and the accumulated pebble burnup, would appear to be sufficient to give accurate cross-sections useful for rapid depletion analysis.

Finally, we conclude with recommendations for future feature development to facilitate higher-fidelity analysis of the core behavior, including the transient evolution from the initial fresh core to the equilibrium compositions, as well as enhancements to the ORIGAMI interface to ORIGEN to facilitate rapid, wholecore evaluations of core inventories from depletion calculations. 


\section{INTRODUCTION}

Pebble-bed reactors, fueled by graphite-coated, spherical fuel elements composed of doubly heterogeneous tri-structural oxide (TRISO) fuel kernels, represent a radical departure from conventional light water reactors in terms of operational characteristics. Among these differences, modular pebble bed reactors (PBRs) (including variants such as the South African PBMR-400 design, the Chinese HTR-10, and the German Arbeitsgemeinshaft Versuchsreaktor, or AVR) all function at high operating temperatures $\left(800-950{ }^{\circ} \mathrm{C}\right)$ with fuel pebbles that are continuously circulated through the core over multiple passes to reach relatively high burnup (on the order of 90-100 $\frac{\mathrm{GWd}}{\mathrm{MTIHM}}$ ) [1]-[3]. PBR systems thus require fundamentally different approaches to neutronics and source term analysis, in particular accounting for the nonstationary nature of the fuel, wherein the axial transit of the fuel pebble results in exposure to substantial swings in the neutron flux spectrum characteristics (i.e., average lethargy).

The goal of this analysis is to establish first-order effects on discharge isotopics for evaluating pebble depletion cases, with the intent of creating standardized one-group ORIGEN reactor data libraries to facilitate rapid depletion calculations. Essential to the process of creating accurate, one-group depletion libraries is characterizing the magnitude of various effects within the core on the discharge isotopic composition, ranging from proximity to graphite reflector layers (producing an overall softer spectrum) to the time-dependent power resulting from the axial transit through the core.

To perform this analysis, we relied on the PBMR-400 international reactor physics benchmark [2], [3] using Monte Carlo transport and depletion capabilities present in the latest pre-release version of SCALE 6.3 [4]. While it is expected that individual design aspects may vary among PBRs, many of the most important phenomena for analysis of this class of reactors are shared among these designs: high-temperature operation, high burnup discharge, graphite moderation, TRISO fuel particles embedded within fuel pebbles which experience multiple passes through the core. Thus, it is anticipated that key analysis principles developed here would likewise apply to other pebble bed-based variants.

The remainder of this section thus consists of a review of prior analyses conducted with PBR-class designs and a brief overview of the computational methods in SCALE to model "doubly heterogeneous fuel"a term used extensively within SCALE to denote a fuel element that does not contain a homogeneous fuel mixture but rather a heterogeneous mixture of discrete fuel particles within a larger medium. For the PBR, the fuel element is a pebble and the fuel mixture is the thousands of discrete TRISO fuel particles interspersed throughout the pebble's graphite matrix. Following chapters then introduce the PBMR-400 benchmark and cases considered to provide validation of the model to be used, followed by analysis of neutronic characteristics at the core level (including axial and radial power distributions and neutron energy flux profiles).

The core-level characteristics established are used to inform single-pebble depletion analysis by providing a means of estimating the effects of nonuniform power histories, as well as differences in the neutron spectrum arising as results of changing axial and radial locations within the core over the pebble lifetime. This component will focus on macroscopic-level characteristics of depletion, including the change in observable characteristics such as decay heat, activity, and isotopic distribution within the fuel resulting from coreaveraged vs. regional features.

Following this, we broaden the analysis to consider the effects of including nearest neighbors to estimate average neutron flux characteristics for depletion, in order to determine their effects on one-group crosssections and ultimately calculated isotopic inventories. 
Finally, this report presents summary findings and recommended strategies for developing accurate ORIGEN reactor libraries for PBMR source term analysis and the extension of these depletion capabilities to the ORIGAMI interface to ORIGEN.

\subsection{PRIOR ANALYSES USING SCALE OF PEBBLE-BED TYPE SYSTEMS}

Although an exhaustive review of previous computational studies of PBR neutronic and thermal hydraulic behavior is beyond the scope of this report, numerous studies have been performed to characterize the physics environment of PBR-type systems, including the HTR-10 and PBMR-400 benchmarks. This section will present a brief summary of some of the findings from those studies that are most relevant to the analysis discussed in this report.

\subsubsection{HTR-10 BENCHMARK}

Among the most closely related studies to this work are prior benchmarking studies performed using SCALE to evaluate the HTR-10 initial core critical benchmark [5], [6]. This study provided validation of the newlydeveloped SCALE capability for accurate self-shielding of fuel in doubly heterogeneous systems. The chief figure of merit in this validation study was in the k-eigenvalue $\left(\mathrm{k}_{\mathrm{eff}}\right)$ for the initial critical core configuration. No studies on depletion or core power distributions were documented in the available reports.

To perform this validation, the HTR-10 initial core critical benchmark model from the International Handbook of Evaluated Reactor Physics Benchmark Experiments (IRPhE-Handbook) was created with SCALE 6.0 and compared with the reported benchmark model created using MCNP [7]. The SCALE/CSAS k $\mathrm{eff}$ results with KENO-VI Monte Carlo transport and ENDF/B-VII.0 cross-sections were consistent with the benchmark reference MCNP, with a difference of $-73 \pm 34 \mathrm{pcm}$ [6]. Both SCALE and MCNP showed a consistent positive reactivity bias of $(1.4 \pm 0.4) \%$ compared to the actual system [6].

The HTR-10 design shares a number of similar features with the PBMR-400 benchmark used for the current study. Like the PBMR-400 benchmark, the HTR-10 uses graphite-coated, spherical fuel elements with TRISO fuel particles of approximately the same dimensions as the those in the PBMR (with a pebble radius of $3 \mathrm{~cm}$ ), which are placed at the top of the core at the beginning of the pebble cycle, continuously circulating downward to the core discharge outlet at the end of cycle. Neutron moderation is provided by outer graphite reflector regions. Notable differences in the HTR-10 design, however, include its cylindrical core design (unlike that of the PBMR-400, which is composed of an annular core region with both an inner and an outer graphite reflector) and a higher average pebble enrichment $\left(17 \mathrm{w} /{ }^{235} \mathrm{U}\right.$ for the HTR-10 compared with $9.6 \mathrm{w} / \mathrm{o}$ for the PBMR-400).

\subsubsection{HTR-PROTEUS BENCHMARK}

The PROTEUS facility is a zero-power research reactor located at the Paul Scherrer Institut in Switzerland that is used for reactor design studies. Key design features of PROTEUS include a flexible layout which can be reconfigured to represent different reactor types [5] $]^{1}$. From 1992 to 1996, PROTEUS was configured as a pebble bed critical facility (designated HTR-PROTEUS) to study PBR-based configurations. During this period, reactor physics experiments were performed on 13 critical configurations of the core that are captured by the benchmark reported in [5].

For these experiments, the pebble configuration was similar in dimensions to the HTR-10 and PBMR-400 benchmarks, with a $2.35 \mathrm{~cm}$ spherical fuel region (containing $9,394 \mathrm{UO}_{2}$ fuel kernels) in a $3 \mathrm{~cm}$ graphite matrix at an enrichment of $16.7 \mathrm{w} / \mathrm{.}$.

\footnotetext{
${ }^{1}$ Thus, much like the HTR-10 benchmark, the effective core shape is a right cylinder filled with packed spheres.
} 
Ten deterministic pebble arrangements within the core were evaluated as part of these experiments, in addition to three configurations consisting of RCP arrangements. Of these, the ten deterministic arrangements, four hexagonal close-packed (HCP) and six columnar hexagonal point-on-point (CHPOP) were modeled using SCALE [5]. A summary of the configurations evaluated is presented as Table 1.

Table 1. Deterministic pebble configurations modeled with SCALE for the HTR-PROTEUS benchmarks (adapted from [5]).

\begin{tabular}{|c|c|c|c|c|}
\hline Core ID & State(s) & Packing & Pebble configuration(s) & $\begin{array}{l}\text { Ratio of fuel to } \\
\text { moderator pebbles }\end{array}$ \\
\hline 1 & State 1 & $\mathrm{HCP}$ & 22 layers & 2.00425 \\
\hline $1 \mathrm{~A}$ & $\begin{array}{l}\text { State } 1 \\
\text { State } 2\end{array}$ & $\mathrm{HCP}$ & $\begin{array}{l}21 \text { layers } \\
\text { Same as State } 1 \text { but with slightly different } \\
\text { control rod locations }\end{array}$ & 2.00445 \\
\hline 2 & State 1 & $\mathrm{HCP}$ & $\begin{array}{l}16 \text { layers } \\
17^{\text {th }} \text { layer: moderator pebbles only }\end{array}$ & 2.00425 \\
\hline 3 & State 1 & $\mathrm{HCP}$ & $\begin{array}{l}17 \text { layers } \\
18^{\text {th }} \text { layer partially filled } \\
327 \text { cylindrical polyethylene rods }\end{array}$ & 2.0045 \\
\hline 5 & $\begin{array}{l}\text { State } 1 \\
\text { State } 2\end{array}$ & CHPOP & $\begin{array}{l}22 \text { layers } \\
23^{\text {rd }} \text { layer }\end{array}$ & $\begin{array}{l}2.000378 \\
0.6188\end{array}$ \\
\hline 6 & $\begin{array}{l}\text { State } 1 \\
\text { State } 2\end{array}$ & CHPOP & $\begin{array}{l}22 \text { layers } \\
23^{\text {rd }} \text { layer } \\
654 \text { triangular polyethylene rods }\end{array}$ & $\begin{array}{l}2.000378 \\
0.6188\end{array}$ \\
\hline 7 & State 1 & CHPOP & $\begin{array}{l}17 \text { layers } \\
18^{\text {th }} \text { layer } \\
654 \text { triangular polyethylene rods }\end{array}$ & $\begin{array}{l}1.99951 \\
0.5628\end{array}$ \\
\hline 8 & State 1 & CHPOP & $\begin{array}{l}22 \text { layers } \\
23^{\text {rd }} \text { layer } \\
654 \text { triangular polyethylene rods }\end{array}$ & $\begin{array}{l}2.0378 \\
0.6188\end{array}$ \\
\hline 9 & $\begin{array}{l}\text { State } 1 \\
\text { State } 2\end{array}$ & CHPOP & $\begin{array}{l}27 \text { layers } \\
28^{\text {th }} \text { layer of pure moderator }\end{array}$ & $\begin{array}{l}0.998565 \\
0.929744\end{array}$ \\
\hline 10 & State 1 & CHPOP & $\begin{array}{l}27 \text { layers } \\
654 \text { cylindrical polyethylene rods }\end{array}$ & 0.998565 \\
\hline
\end{tabular}

For benchmark comparisons with MCNP5, the analysts used ENDF/B-VI cross-sections to improve consistency between codes. The difference in $\mathrm{k}_{\mathrm{eff}}$ between the SCALE and MCNP models for HCP arrangements was cited as $252 \pm 93 \mathrm{pcm}$, with a maximum difference of $353 \pm 84 \mathrm{pcm}$ [5]. For the columnar hexagonal point-on-point (CHPOP) configurations, an average difference in $\mathrm{k}_{\mathrm{eff}}$ was $422 \pm 93 \mathrm{pcm}$, with a maximum difference of $667 \pm 82 \mathrm{pcm}$ [5].

When the reported IRPhE-Handbook benchmark values were compared with calculations using SCALE with a 238-group ENDF/B-VII.0 library, an average $\mathrm{k}_{\mathrm{eff}}$ difference of $797 \mathrm{pcm}$ was observed, with a maximum deviation of $1302 \pm 81 \mathrm{pcm}$ [5]. In all cases observed, SCALE exhibited an overestimation of the system $\mathrm{k}_{\mathrm{eff}}$. 


\subsection{SCALE COMPUTATIONAL METHODS}

This section provides a brief review of the computational methods employed for the analysis performed in this report, including SCALE capabilities used for treatment of doubly heterogeneous TRISO fuel as well as an algorithm developed to facilitate the efficient generation of hexagonal pebble arrays for an annular volume. Unless otherwise noted, all criticality and flux distribution calculations for this analysis were performed using multi-group Monte Carlo transport within the CSAS criticality sequence using KENO [8] and/or Shift [9].

\subsubsection{DOUBLY HETEROGENEOUS FUEL TREATMENT METHODS IN SCALE}

Traditional LWR fuel represents a single level of heterogeneity, i.e. within each material in the reactor (coolant, cladding, fuel), it may be treated as a homogeneous mixture. For example, we do not model the grains in $\mathrm{UO}_{2}$ or $\mathrm{Gd}_{2} \mathrm{O}_{3}$, simply assume uniform initial compositions. The SCALE/XSProc module is used to determine the correct spatial and energy self-shielding effects necessary for accurate multi-group transport [10].

Fuels like TRISO-impregnated pebbles and prismatic block fuel employed in HTGR systems present a second level of heterogeneity. Inside this type of fuel, there are flux depressions at each individual TRISO and moderation in the graphite matrix that requires special treatment. Neglecting this second level of heterogeneity by homogenizing the fuel region based on mass/volume preservation results in substantial underestimates of reactivity on the order of several thousand pcm [11].

The SCALE/XSProc self-shielding module (specifically the CENTRM component) accounts for randomly distributed fuel particles using Equivalence Theory [10], [12]. First, the flux spectrum in spherical unit cells representing an infinite array of fuel grains contained within the graphite matrix is calculated using CENTRM [12]. This spectrum is then used to calculate disadvantage factors to create reaction-rate preserving equivalent cross-sections for a "homogenized" mixture in the pebble interior. A second XSProc-CENTRM calculation is used to determine the flux spectrum which includes the effect of materials outside the pebble/prismatic block. For example, the influence of an adjacent pebble, control element, or reflector. for the macroscopic lattice (i.e., pebble-to-pebble or a prismatic block matrix) to calculate a pointwise flux used as a weighting function for calculating multi-group cross-sections [12].

This Equivalence Theory-based method has been extensively tested, including direct comparisons of the multi-group treatment to Monte Carlo calculations using randomly distributed fuel particles [11] and has been used for prior SCALE analyses of HTGR systems [13], [14]. In general, the errors associated with this method, compared with those from direct calculation using continuous energy Monte Carlo with explicit fuel grain representations, have been found to be small (on the order of $100 \mathrm{pcm}$ or less) [11].

\subsubsection{SHIFT CAPABILITIES SPECIFIC TO TRISO FUEL PARTICLES}

Shift is a relatively new Monte Carlo transport code which will eventually replace KENO and Monaco in SCALE. Shift was designed for high-performance computing, including massively parallel operation with GPUs [9]. Shift will be available in the next major SCALE release, version 6.3. This section will provide additional background on features unique to Shift that will be used in this analysis in addition to the doubly heterogeneous methods already outlined previously.

One particularly important feature for single-pebble depletion analysis is Shift's capability to represent random particle distributions within a fuel pebble which can be used to benchmark the SCALE/XSProc methodology recommended for fast, production calculations. Array-based placement of kernels have been studied but compared to the more realistic random placement, a negative $\mathrm{k}_{\mathrm{eff}}$ bias of a few hundred pcm has been noted [14]. 
Shift introduces two stochastic fuel particle generation capabilities for use with continuous energy calculations: distributing fuel particles throughout the entire fuel domain and distributing within a user-defined mesh using a fixed number of particles per mesh cell, but within each mesh cell placed randomly [14]. The latter method represents a compromise that preserves the random spatial distribution of fuel particles yet affords greater performance by reducing the number of surfaces that must be tracked within an individual mesh voxel between neutron interactions [14].

The memory and runtime required for continuous energy Monte Carlo with explicit representation of TRISO fuel particles is impractical for full-core models, however this approach will be used with single-pebble depletion models to assess the multi-group method bias.

\subsection{PRIOR APPROACHES TO PEBBLE-BED REACTOR PHYSICS ANALYSIS}

The unique challenges of flowing-pebble systems, particularly for systems employing mutli-pass fuel cycles, serve to frustrate traditional reactor physics analysis methods, namely in that these systems violate many cardinal assumptions underlying the validity of tools typically used for LWR analysis. Some of these include:

- Continuous movement of pebbles during operation. Regardless of whether the reactor fuel cycle is One Through Then Out (OTTO) or MEDUL (translated as "multiple pass-through"), flowing-pebble systems introduce the challenge of capturing the continuous motion of pebbles through the core during irradiation. This includes capturing variation in the neutron spectrum and power profile as a result of changing position. Moreover, in the case of MEDUL cycles, this likewise includes capturing the continuous recirculation and mixing of pebble batches as they are recycled from the bottom of the core.

- Large problem domain relative to neutron mean free path. In most LWR assemblies, the relatively high efficiency of water as a moderator results in a neutron mean free path that is much smaller than the typical assembly unit size, thus limiting the impact of neighboring assemblies upon the accuracy of the transport and depletion solution. As such, single-assembly models with reflective boundary conditions perform quite well at capturing depletion behavior. By contrast, the relatively small size of the fuel pebbles in PBR systems (typically on the order of $3 \mathrm{~cm}$ diameter), combined with the relatively long neutron mean free path for graphite-moderated systems (wherein $L \approx 59 \mathrm{~cm}$ ), mean that neighboring pebbles within the core exhibit significant influence over the spectral characteristics of a given pebble and thus must be explicitly accounted for within models.

- Strong temperature feedbacks. A characteristic feature of graphite-moderated systems such as the PBMR is a strong coupling of reactivity to temperature. For the PBMR, this specifically translates to a strong negative reactivity feedback from elevated temperatures, touted as an inherent safety feature. However, this also introduces a challenge for modeling in that accurately capturing spectral shifts arising from spatial temperature variations requires explicit coupling of reactor physics to thermal hydraulics modeling. While for steady-state, equilibrium calculations this can be bypassed by using $a$ priori information about temperature distributions, its effects are sufficiently pronounced as to require its careful inclusion in reactor models.

In the following sections, we will present a summary of historical approaches to capturing pebble-bed reactor behavior, including how the modeling challenges outlined above have been addressed.

\subsubsection{VSOP}

The VSOP code system is a comprehensive physics package for the simulation of pebble-bed HTGRs, combining neutron transport (via P1 diffusion theory) with cross-section processing, thermal hydraulics, and 
fuel depletion, shuffling, and management [15]. VSOP's basic method of solution shares many features with conventional LWR core simulator tools, specifically in its approach to establishing region-wise flux shapes to derive few-group cross-sections and leakage corrections such to produce a core-wide power profile.

VSOP handles the challenge of treating continuously-shuffling fuel by iteratively solving the coupled physics| relationships (neutron diffusion, fuel depletion, and thermal hydraulics) for a given time step, illustrated as Figure 1. This depletion feedback loop is repeated until the core reaches a pre-set value of $\mathrm{k}_{\text {eff }}$, whereupon the fuel is shuffled and the core physics calculation is repeated [15].

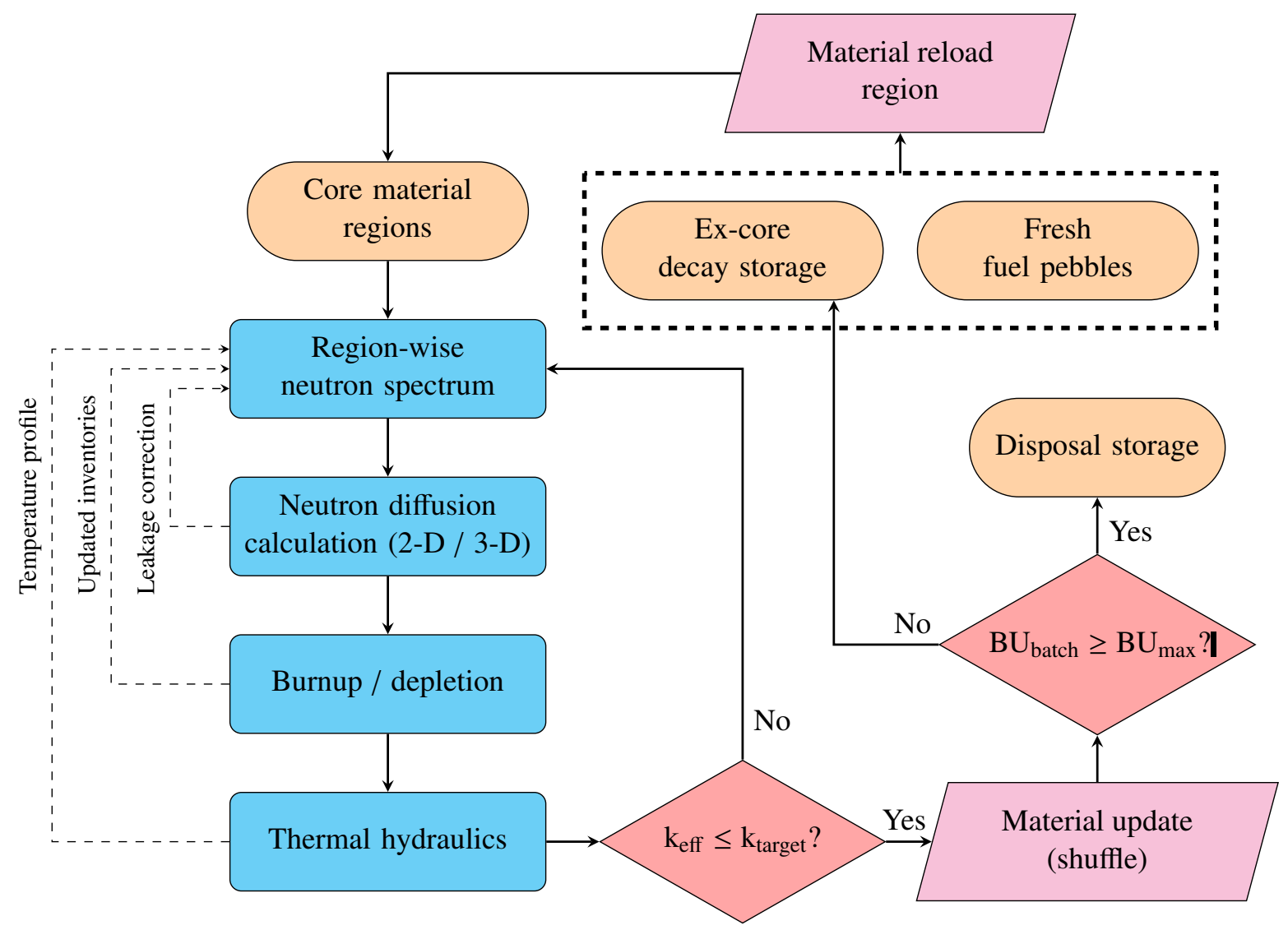

Figure 1. VSOP calculation flow for MEDUL (multi-pass) cores [15]

VSOP's basic unit of materials tracking within reactor calculations is that of a "batch," effectively a cohort of pebbles entering the core at the same time and having experienced the same number of passes. Within a physical region of the core, pebbles from multiple batches (i.e., comprising a range of burnups and compositions) are presumed to experience the same local neutron flux. Hence, fine-group cross-sections are calculated for each fuel pebble within a physical region (allowing for separate depletion of individual pebbles from multiple batches); the compositions within the region are then volume-weighted to produce a homogenized region-wise material mixture. The homogenized region mixtures are then used to produce few-group cross-sections for solving the 2-D / 3-D core diffusion calculation giving the core spatial flux distribution and leakage correction terms which are subsequently applied back to fine-group cross-sections used for depletion [15]. Within a region, fuel elements are individually depleted based on the local regionwise flux (with corresponding leakage corrections) and the step time. VSOP likewise handles out-of-core fuel management and decay, including separating pebbles that have reached the discharge burnup limit and calculating reload batches of new and recycled pebbles. 
Diffusion calculations in VSOP rely on ENDF/B-V and JEF-1 cross-sections, using the 68-group GAM-I library for fast neutrons (ranging from $10 \mathrm{MeV}$ through $0.414 \mathrm{eV}$ ), with cross-sections for 171 materials and six additional short-lived heavy metal isotopes ${ }^{233} \mathrm{Th},{ }^{239} \mathrm{U},{ }^{238} \mathrm{~Np},{ }^{240} \mathrm{~Np},{ }^{243} \mathrm{Pu}$, and ${ }^{244} \mathrm{Am}$ ) which do not have explicit capture cross-sections within the library but are considered for their contributions to build up of actinide inventories [15]. Treatment of thermal-energy absorption is treated by a 30-group THERMOS library (ranging from $10^{-5}$ to $2.05 \mathrm{eV}$ ), which includes absorbers from the GAM-I library as well as scattering information for an additional 38 nuclides (consisting of isotopes of hydrogen, deuterium, oxygen, carbon, and beryllium).

Depletion calculations in VSOP use the matrix exponential solver based upon the same solver subroutine historically used in ORIGEN [15], [16]. For the iterative in-core solution, VSOP relies upon the limited subset of materials in the GAM-I and THERMOS libraries described above, however users can also perform more detailed depletion calculations using stored inventories and power histories (provided by VSOP in terms of the ORIGEN-JÜL-II variant of ORIGEN) [15], [17].

One of the main advantages of the VSOP approach is in its ability to establish the time-dependent evolution of the core from initial fresh conditions to the equilibrium state. This generally affords VSOP maximum flexibility in simulating PBR cores at the cost of additional computational time. Notably, newer codes designed to simulate HTGR systems such as the HINT package largely reproduce much of the same computational flow as VSOP with different physics implementations and more modern nuclear data [18].

\subsubsection{PEBBED}

The PEBBED code for HTGR simulation was developed by Idaho National Laboratory (INL) in 1999. Unlike VSOP, PEBBED does not calculate the intermediate ("running-in") states of the core, but rather is designed to converge directly upon the equilibrium core flux and composition [19], [20]. PEBBED does this by imposing an axial boundary condition of the set of pebbles entering the top of the core and taking a userdefined "initial guess" flux spectrum to iteratively solve for the equilibrium flux profile and compositions using a known fuel recirculation pattern (i.e., a matrix describing the weighted probability of pebbles exiting and entering the system based on the radial position and burnup, referred to as the "recirculation matrix") [19], [21], [22]. In effect, PEBBED solves a modified version of the neutron diffusion equation (containing an additional advection term), expressed as Eq. (1) [22].

$$
\begin{aligned}
\frac{\partial N_{k}(r, z, t)}{\partial t}+\frac{\partial N_{k}(r, z, t)}{\partial z} v_{z}(r, z) & =\phi(r, z, t) \sum_{i=l}^{m} N_{i}(r, z, t) \sigma_{f}^{i} y_{i \rightarrow k}+\phi(r, z, t) \sum_{s=r}^{q} N_{s}(r, z, t) \sigma_{a}^{s} \gamma_{s \rightarrow k} \\
& +\sum_{j=n}^{p} N_{j}(r, z, t) \lambda_{j} \alpha_{j \rightarrow k}-\lambda_{k} N_{k}(r, z, t)-\phi(\vec{r}, t) N_{k}(r, z, t) \sigma_{a}^{k}
\end{aligned}
$$

In converging upon the "equilibrium" solution, PEBBED effectively assumes the leftmost term in Eq. 1 converges to zero (i.e., no change in the nuclide inventory with respect to time) [22]. PEBBED's solution flow is otherwise similar to VSOP, using few-group macroscopic cross-sections for core-wide diffusion calculations to update local microscopic cross-sections for depletion, performing an iterative calculation on the neutron source and $\mathrm{k}_{\mathrm{eff}}$ until convergence is reached [21]. Like VSOP, PEBBED explicitly accounts for thermal feedbacks via detailed thermal hydraulic treatments.

PEBBED assumes a strictly axial flow of pebbles through the core [22], although differential flow velocities (as a function of radial position) are accounted for. Notably, this assumption of strictly-axial flow is a default 
for VSOP, however the latter package allows for user-specification of specific flow trajectories based upon external information [15].

PEBBED's physics treatment for depletion rely upon a 167-group spectrum, using a Bonderenko Method for unresolved resonance treatment [22], similar to the technique used in BONAMI in SCALE [10]. Treatment of the fuel double heterogeneity is treated using separate Dancoff factors for the TRISO fuel microparticles and the larger fuel pebbles [22]. Depletion calculations via Laplace transform of the Bateman Equations, requiring a linearization of the burnup chains [19].

In general, PEBBED is capable of producing reasonable convergence to equilibrium inventories calculated by VSOP for longer irradiation periods, with the largest differences appearing in the higher transuranic species (e.g., ${ }^{242} \mathrm{Cm},{ }^{244} \mathrm{Cm}$, etc.) and shorter-lived fission products (including those relevant to severe accident source term analysis, such as ${ }^{109} \mathrm{Ag}$ and ${ }^{113} \mathrm{Cd}$ ) [19]. Largely the differences appear to arise from the user of the linearized burnup chains (i.e., from the use of the Laplace transform) compared to the use of the matrix exponential solver.

\subsection{OVERVIEW OF THE PBMR-400 BENCHMARK PROBLEM}

The PBMR-400 design was originally considered for development by the South African utility ESKOM as part of a larger industrial consortium, PBMR Ltd., which also included the South African Government and British Nuclear Fuel [2]. While a reactor was never constructed, sufficient design details of the PBMR exist such as to serve as a useful verification test case.

The PBMR-400 design is based upon a scaled-down version of prior high-temperature gas-cooled reactors, designed to capture advantages of prior PBR designs such as the German Arbeitsgemeinshaft Versuchsreaktor (AVR) as well as safety features of HTGR designs such as the Fort Saint Vrain prismatic block HTGR (United States) and the THTR-300 (300 MW thorium high temperature reactor, Germany) [2]. The goal of the PBMR-400 design was to combine the excellent fission product retention capabilities demonstrated in TRISO-based spherical pebble fuel with the high-temperature outlet and high-efficiency Brayton cycle employed in HTGR designs [2]. Designed for 400 megawatts thermal (MWt) power output, the core consists of approximately 452,000 graphite-coated fuel pebbles, each containing approximately $15,000 \mathrm{UO}_{2} \mathrm{TRISO}$ fuel particles [2], [3]. The core consists of an annular region between two cylindrical graphite blocks that serve as neutron moderators (an "inner" and "outer" reflector with radii $100 \mathrm{~cm}$ and $185 \mathrm{~cm}$, respectively) with an active core height of approximately $10.117 \mathrm{~m} \mathrm{[2],} \mathrm{[3].} \mathrm{Primary} \mathrm{control} \mathrm{through} \mathrm{the} \mathrm{reactor} \mathrm{control}$ system (RCS) is provided by 24 boral carbide $\left(\mathrm{B}_{4} \mathrm{C}\right)$ rods oriented equidistantly around the core in the outer reflector region at a pitch circle diameter $(\mathrm{PCD})$ of $3.974 \mathrm{~m}$. A summary of the major dimensional features used for modeling is presented as Table 2.

Although the computational benchmark model [2] makes a number of simplifications to the PBMR core geometry (e.g., homogenization of the fuel region, homogenization of the reflector graphite and graphite riser channels, treatment of the radial control rods as "gray curtain" region surrounding the core), many geometric details of the reactor core are included within the SCALE models used for this analysis. They include explicit treatment of the pebble geometry within the core and explicit modeling of the discrete control rod elements, the gas riser void spaces (as specified in the benchmark) within the reflector regions, and the outer core structural materials (e.g., the reactor pressure vessel (RPV)).

The PBMR-400 benchmark problem specification consists of a number of separate conditions, including both neutronic and thermal-hydraulic calculations. A brief summary of the subset of cases used for verification studies in this report is presented as Table 3.

Simplifications introduced into the SCALE model of the PBMR-400 benchmark include not explicitly modeling of the pebble discharge funnel regions (rather, it simply treats the core effectively as a cylindrical 
Table 2. Key physical dimensions of the PBMR-400 reactor [2], [3].

\begin{tabular}{ll|ll}
\hline Fuel pebble & & TRISO fuel kernel & \\
\hline Fuel region radius & $2.5 \mathrm{~cm}$ & $\mathrm{UO}_{2}$ density & $10.4 \frac{\mathrm{g}}{\mathrm{cm}^{3}}$ \\
Graphite outer layer thickness & $5 \mathrm{~mm}$ & Fuel kernel diameter & $500 \mu \mathrm{m}$ \\
Fuel pebble radius & $3.0 \mathrm{~cm}$ & Fuel coating layers & $\mathrm{C} / \mathrm{PyC} / \mathrm{SiC} / \mathrm{PyC}$ \\
$\mathrm{UO}_{2}$ per pebble & $9 \mathrm{gHM}$ & Coating thicknesses $(\mu \mathrm{m})$ & $95 / 40 / 35 / 40$ \\
TRISO particles per pebble & 15,000 & Coating densities $\left(\frac{\mathrm{g}}{\mathrm{cm}^{3}}\right)$ & $1.05 / 1.90 / 3.18 / 1.90$ \\
\hline Core \& Reflector & & Control & \\
\hline Active core height (effective) & $11.0 \mathrm{~m}$ & RCS material & $\mathrm{B}_{4} \mathrm{C}$ \\
Number of fuel pebbles & $\approx 452,000$ & RCS B ${ }_{4} \mathrm{C}$ density & $2.2 \frac{\mathrm{g}}{\mathrm{cm}^{3}}$ \\
Pebble packing fraction & $61 \%$ & Number of RCS rods & 24 \\
Central reflector outer radius & $1.0 \mathrm{~m}$ & PCD of control rod borings & $3.974 \mathrm{~m}$ \\
Outer reflector inner radius & $1.85 \mathrm{~m}$ & RCS rod radius & $5.0 \mathrm{~cm}$ \\
Outer reflector outer radius & $2.75 \mathrm{~m}$ & & \\
Reflector graphite density & $1.8 \frac{\mathrm{g}}{\mathrm{cm}}$ & & \\
Reflector boron impurity & $1 \mathrm{ppm}$ & & \\
\hline
\end{tabular}

Table 3. Selected PBMR-400 neutronic benchmark problems, from [2].

\begin{tabular}{lllll} 
Case & Description & Fuel & Temp(s) (K) & RCS \\
\hline F-2(i) & Fresh core, CZP & $\begin{array}{l}1 / 3 \text { fresh fuel }\left(9 \text { gHM, } 5.768 \text { w } /{ }^{235} \mathrm{U}\right) \\
2 / 3 \text { graphite “dummy" pebbles }\end{array}$ & 300 & No \\
F-2(ii) & Fresh core, HZP & & & No \\
\hline E-2(i) & Equilibrium core, CZP & Equilibrium compositions (ND-Set3) & 300 & $2.285 \mathrm{~m}$ \\
E-2(ii) & Equilibrium core, HZP & & $600,900,1200$ & $2.285 \mathrm{~m}$ \\
\hline
\end{tabular}

annulus over the entire effective axial length of the core). It is anticipated that this approach will have negligible effects upon evaluated characteristics of interest, such as the power profile and system characteristics such as $k_{\text {eff }}$ and energy of the average lethargy causing fission (EALF). The PBMR-400 benchmark problem specifies a core loading of approximately 451,530 spheres loaded in the core. When the sphere packing algorithm is used, the vertex tolerance factors were adjusted to yield a total of 451,616 spheres in the core, a difference of 86 spheres (a $0.019 \%$ difference). 


\section{PBMR-400 MODEL VERIFICATION}

The following sections discuss the verification exercises conducted with the PBMR-400 benchmark, using a pre-release version of SCALE 6.3, to verify the correctness of the PBMR-400 models used for this analysis, noting any biases present. This approach consists of three separate benchmarks: a single, reflected pebble (Section 2.1); a full core at fresh, starting conditions (Section 2.2.1); and finally a full core at equilibrium power and compositions (Section 2.2.2). In addition to their validation function, these models serve as a useful tool for evaluating changes to core characteristics (such as the power profile) moving from the initial startup conditions to equilibrium. Further, the differences in model scope serve as a starting point to explore the effects of model scale on calculated depletion results at the pebble level (informing the development of ORIGEN libraries for pebble depletion).

\subsection{SINGLE, REFLECTED PEBBLE MODEL}

Figure 2 illustrates an infinitely-reflected, single-pebble SCALE model of the PBMR fuel pebble used for intra-pebble flux and depletion studies. The single-pebble model effectively assumes an infinite lattice of fresh pebbles (at an enrichment of $5.76 \mathrm{w} /{ }^{235} \mathrm{U}$ ) with a $2.5 \mathrm{~cm}$ fuel region composed of 15,000 TRISO fuel particles. The pebble is in a hexagonal prismatic unit cell, thus representing a CHPOP geometry that preserves the core-average pebble pitch and packing fraction.

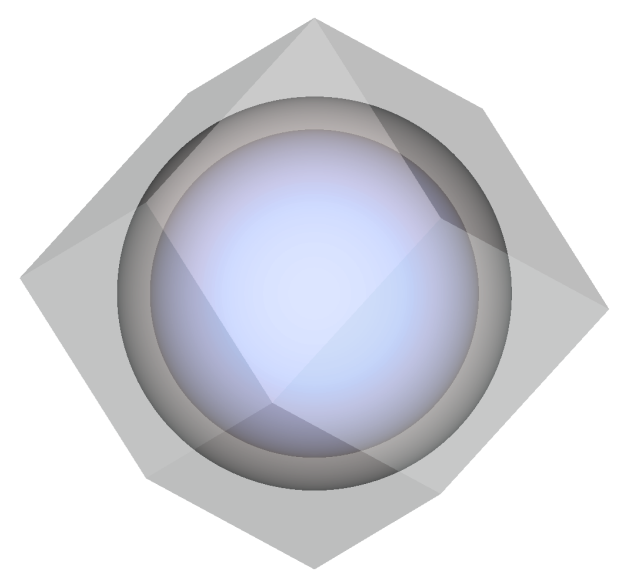

Figure 2. SCALE 6.3 single-pebble reflected model of a fresh PBMR-400 pebble, representing an infinite array of pebbles.

\subsection{FULL-CORE MODELS}

To evaluate the changes in the neutron spectrum characteristics over the irradiation lifetime of a pebble, it is essential to determine the spatial and energy distribution of neutrons throughout the core. The goal of the following sections is therefore to illustrate the SCALE representation of the PBMR-400 core under both the fresh core and equilibrium cycle conditions, as well as the assumptions made in model construction.

For the full-core models, a number of different pebble packing lattice configurations were investigated to attempt to best reproduce the temperature-specific $\mathrm{k}_{\mathrm{eff}}$ benchmarks for the PBMR-400 system. These included a CHPOP stacked hexagonal lattice arrangement and a HCP arrangement using a dodecahedral array; the latter case effectively produces a FCC layout (i.e., giving an equivalent packing density to HCP). Each arrangement was investigated with two variants: one which allowed pebbles to be "clipped" at the core boundary and one which only allowed complete pebbles. 
The CHPOP lattice was not able to reproduce the benchmark packing fraction with either variant. When clipping is not allowed and only full pebbles are included, this arrangement produced a maximum packing fraction of only 57\%. When clipping was allowed, the system mass was preserved but there was not acceptable agreement with the benchmark $\mathrm{k}_{\mathrm{eff}}$ values.

The dodecahedral array of pebbles results in an HCP configuration and thus is able to represent the maximum theoretical packing density of fuel pebbles in the core. To preserve the system packing fraction and heavy metal loading, the lattice pitch may be increased to yield correct number of pebbles and packing fraction, in both the clipped pebble and complete pebble variants. However the clipped configuration produced substantial negative reactivity differences with the benchmark on the order of $-600 \mathrm{pcm}$ for the fresh core at $\mathrm{CZP}$, likely due to the local reductions in the packing fraction at the core boundaries. The most consistent results with the benchmark was found for a clipped pebble model in the close-packed dodecahedral lattice with an effective lattice pitch of $6.4 \mathrm{~cm}$ (i.e., a unit cell radius of $3.2 \mathrm{~cm}$ ), which gives an effective pebble volume fraction of $61 \%$.

\subsubsection{FRESH CORE (CASES F-2(I) AND F-2(II))}

The SCALE model used for analyzing the PBMR-400 fresh core (HCP pebble arrangement) is illustrated as Figure 3. The starting core benchmark consisted of $1 / 3$ fuel pebbles (green) at $5.76 \mathrm{w} / \mathrm{o}$ enrichment and $2 / 3$ graphite "dummy" pebbles, evenly distributed through the core. The SCALE model used discrete pebbles with fuel number densities $N_{h e t}$ as determined from Eq. 2[2].

$$
N_{\text {het }}=N_{\text {hom }}\left(\frac{V_{\text {pebble }}}{V_{\text {kernel }} \cdot n_{k} \cdot p_{f}}\right)
$$

In Eq. 2, $N_{\text {hom }}$ is the homogenized number density of all of the pebble materials (as-specified in the benchmark), $V_{\text {pebble }}$ is the fuel pebble volume, $V_{\text {kernel }}$ is the fuel kernel volume, $n_{k}$ is the number of kernels per fuel pebble $(15,000)$, and $p_{f}$ is the pebble packing fraction in the core $(0.61)$.

This model test was designed to reproduce Cases F-2(i) and F-2(ii) from the PBMR-400 benchmark [2], corresponding to the the core multiplication factors $\mathrm{k}_{\mathrm{eff}}$ at CZP (F-2(i), all materials at $300 \mathrm{~K}$ ) and HZP (F-2(ii), all materials at 600, 900, and $1200 \mathrm{~K}$, respectively). This model series likewise provided a means of evaluating the temperature coefficient of reactivity for the initial, fresh core. Two sets of results from the international benchmark are reported using the nodal diffusion code VSOP—one conducted without a $1 \mathrm{ppm}$ boron impurity in the graphite (South Africa) and one accounting for this impurity (China). The comparison of $k_{\text {eff }}$ values is given as Table 4 .

Table 4. Comparison of fresh core PBMR-400 benchmark model to reported $k_{\text {eff }}$ values from Tables 4.27 and 4.28 of [2], corresponding to cases F-2(i) (CZP), and F-2(ii) (HZP).

\begin{tabular}{r|ll|l|r}
\multirow{2}{*}{ Temp. (K) } & \multicolumn{2}{|c|}{ SCALE 6.3 } & VSOP [2] & \\
& $\mathrm{k}_{\mathrm{eff}}$ & $\sigma_{k}$ & $\mathrm{k}_{\mathrm{eff}}$ & $\Delta \mathrm{k}(\mathrm{pcm})$ \\
\hline 300 & 1.16635 & 0.00011 & 1.16538 & +97.1 \\
600 & 1.11896 & 0.00013 & 1.12424 & -528 \\
900 & 1.09032 & 0.00014 & 1.08875 & +157 \\
1200 & 1.05734 & 0.00012 & 1.06301 & -567 \\
\hline
\end{tabular}

Noteworthy in the comparison of the system isotherm $\mathrm{k}_{\mathrm{eff}}$ values between SCALE and VSOP (Table 4) is the relative lack of a trend; i.e., the difference in $\mathrm{k}_{\mathrm{eff}}$ between SCALE and VSOP oscillates between as high as $+157 \mathrm{pcm}$ and as low as $-567 \mathrm{pcm}$, with no apparent correlation to temperature. 


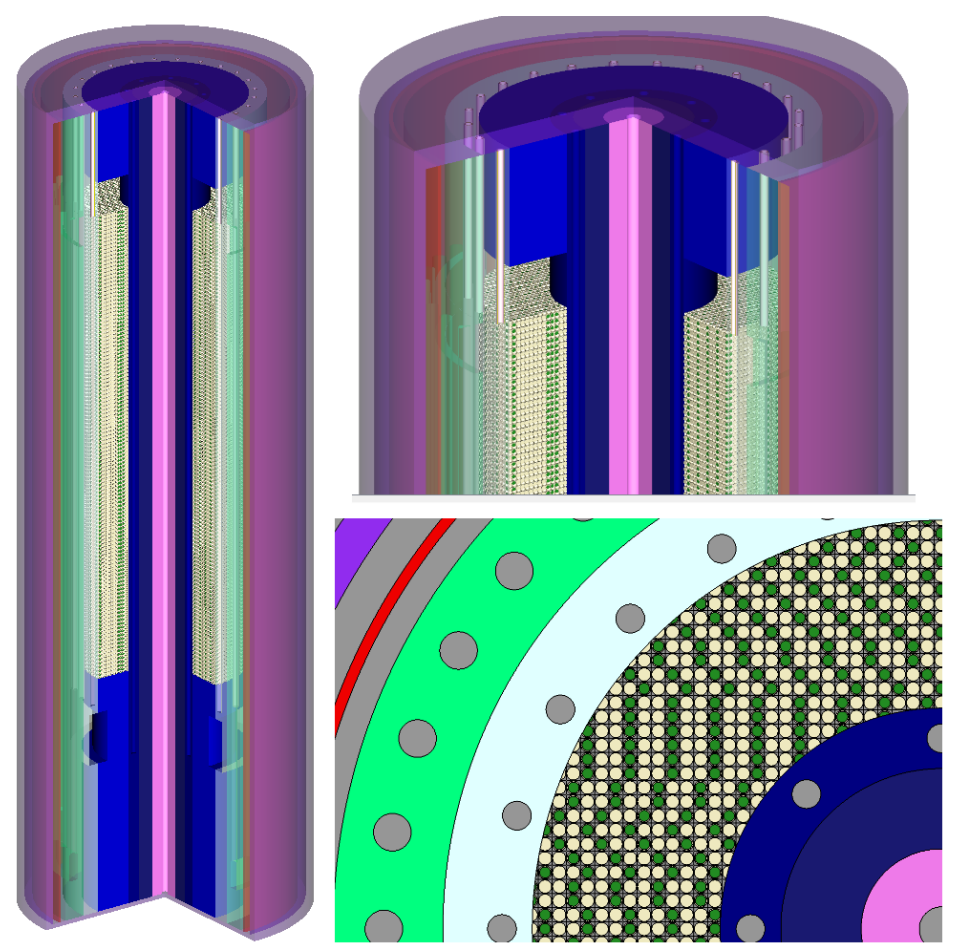

Figure 3. SCALE 6.3 model for the PBMR-400 fresh core, consisting of $1 / 3$ fuel pebbles (green) and $2 / 3$ graphite "dummy" pebbles, HCP (dodecahedral) arrangement; (left) axial cutaway view, (right) axial cut plane views at $z=-100 \mathrm{~cm}$, where $z=0$ represents the top of the active fuel region.

Nonetheless, given that the average $\mathrm{k}_{\mathrm{eff}}$ difference amounts to just over $-210 \mathrm{pcm}$, we can conclude that the SCALE model appears to represent a reasonable implementation of the benchmark problem which can be used for studying the neutronic features of the system.

\subsubsection{EQUILIBRIUM-COMPOSITION CORE (CASES E-1(I) AND E-1(II))}

The SCALE model used for analyzing the PBMR-400 equilibrium-composition core (HCP pebble arrangement) is illustrated as Figure 4. The equilibrium-composition core consists of compositions specified from the ND-Set3 of ref. [3], representing a best estimate of the isotopic distribution within the pebbles at an equilibrium cycle of the core with a fresh pebble enrichment of $9.6 \mathrm{w} /{ }^{235} \mathrm{U}$. ND-Set3 specifies 22 axial regions of equal height and 5 radial regions of equal radius for a total of 110 material zones. Here again the fuel material number densities were reconstructred as per Eq. 2 which preserved heavy metal mass in the core. A second assumption made within this model has been roughly equal distribution of heavy metal mass across radial zones, i.e., each region representing different fuel burnup states is presumed to consist of the same number of pebbles. Thus, the radii used to define the boundaries of the concentric rings making up the different radial fuel zones were calculated to produce rings of roughly equal area.

In contrast to the fresh core comparison (Table 5), the equilibrium-composition core generally showed much larger deviations in $\mathrm{k}_{\mathrm{eff}}$ between CZP and HZP operating conditions, swinging from $+728 \mathrm{pcm}$ in the former case to $-737 \mathrm{pcm}$ in the latter. However, unlike in the fresh core benchmark, no additional data was made available for the intermediate temperatures. Noteworthy for both cases, however, was the higher overall temperature coefficient observed from the discrete pebble model compared with the homogenized system modeled by VSOP. This finding is evaluated in greater detail in the following section. 


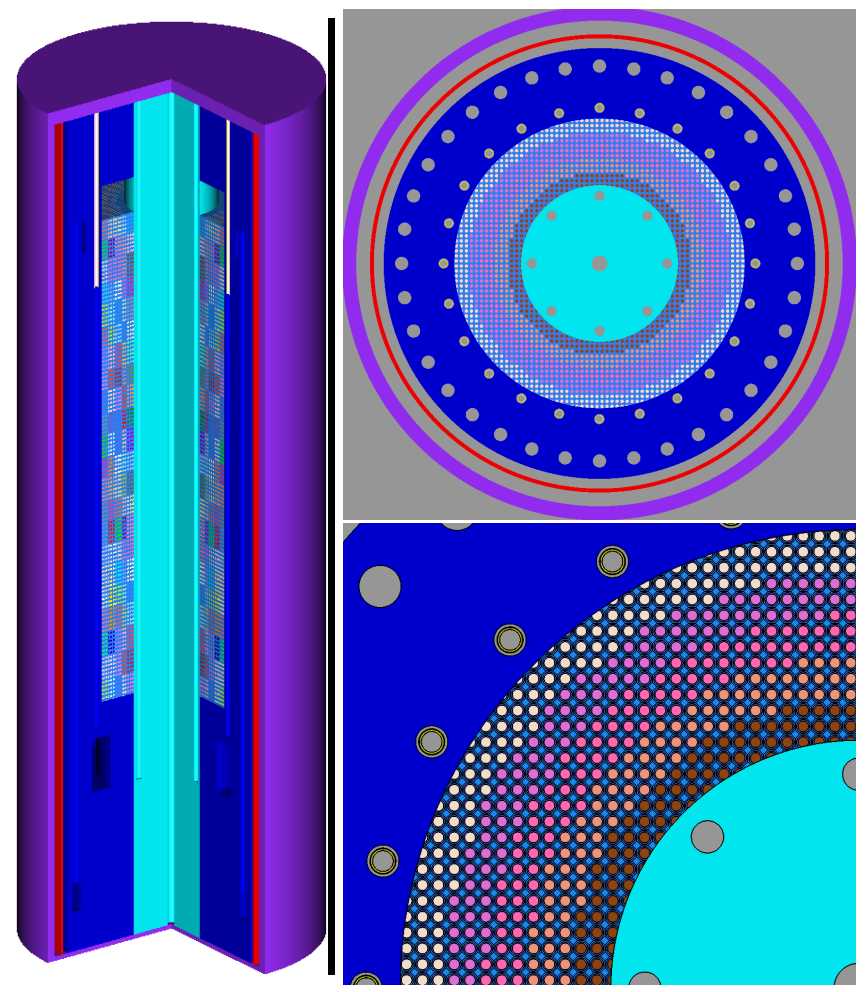

Figure 4. SCALE 6.3 model for the PBMR-400 equilibrium-composition core, dodecahedral lattice / HCP arrangement; (left) axial cutaway view, (right) axial cut plane view at $z=100 \mathrm{~cm}$.

Table 5. Comparison of E-1(i) and E-1(ii) equilibrium-composition core PBMR-400 benchmark model with reported $k_{\text {eff }}$ values from Tables 4.27 and 4.28 of [2].

\begin{tabular}{r|cc|c|c}
\multirow{2}{*}{ Temp. (K) } & \multicolumn{2}{|c|}{ SCALE 6.3 } & VSOP [2] & \\
& $\mathrm{k}_{\mathrm{eff}}$ & $\sigma_{k}$ & $\mathrm{k}_{\mathrm{eff}}$ & $\Delta \mathrm{k}_{\mathrm{eff}}(\mathrm{pcm})$ \\
\hline 300 & 1.01123 & 0.00010 & 1.00395 & +728 \\
600 & 0.99391 & 0.00010 & - & - \\
900 & 0.99188 & 0.00010 & - & - \\
1200 & 0.97870 & 0.00010 & 0.98606 & -736.5 \\
\hline
\end{tabular}




\section{CORE-LEVEL ANALYSIS}

The models developed in Sections 2.2.1 and 2.2.2 afford an analysis of the system-level parameters important for understanding features relevant to both depletion library development and overall core behavior. For example, given the continuous gravity-driven circulation of fuel pebbles through the core, the axially varying power profile was expected to drive the time-dependent pebble irradiation history.

\subsection{SPATIAL DISTRIBUTION OF THE NEUTRON FLUX AND CORE POWER}

The following sections present a comparison of the spatial distribution of the neutron flux and core power for both the fresh and equilibrium-composition cores. This characterization includes an analysis of both the total flux profile within the core and the flux energy profile as a function of axial and radial position.

\subsubsection{TOTAL FLUX AND FISSION SOURCE GRADIENTS OVER THE CORE}

Illustrated in Figure 5 are the total neutron flux profiles across the core axially and radially for the equilibrium-I composition core. (For a corresponding analysis of the fresh core, see Appendix B). Compared with the fresh core scenario, the equilibrium-composition core shows a substantial flux tilt peaking near the top of the core, as opposed to an axially symmetric flux profile. Much of this difference is explained by the inhomogeneity of the equilibrium-composition core; in this case, fresh fuel is loaded at the top and progressively burned as it transits axially downward, thus yielding a pronounced burnup and reactivity gradient. This in turn is expected to influence estimates of the time-dependent power history of individual pebbles as they transit axially through the core, an effect analyzed further in Section 4.2.

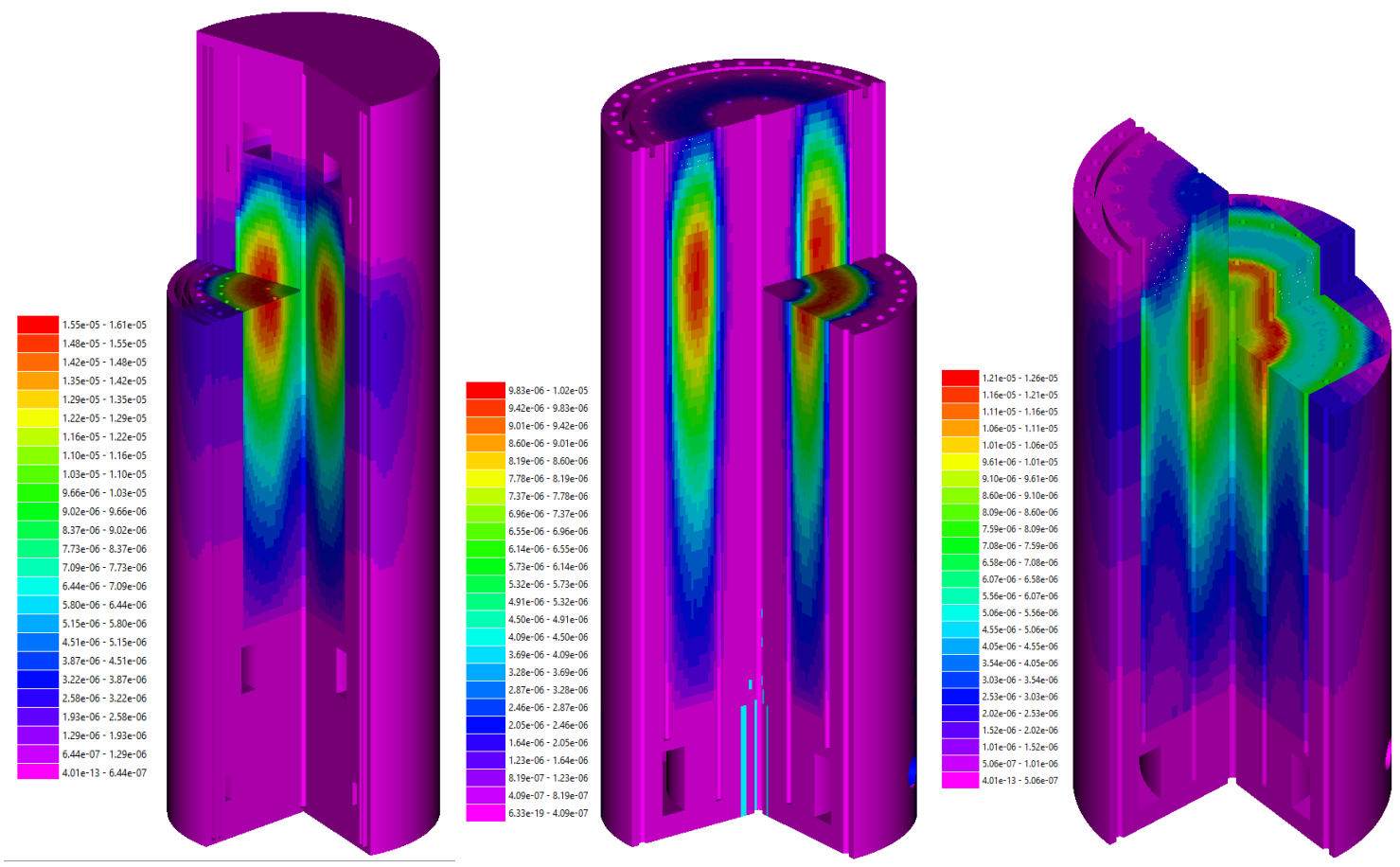

Figure 5. PBMR-400 flux distribution for the equilibrium-composition core; (left) total flux, (center) fast flux $(E>1.86 \mathbf{e V})$, (right) thermal flux $(E<1.86 \mathbf{e V})$. Red indicates regions of highest total neutron flux. 


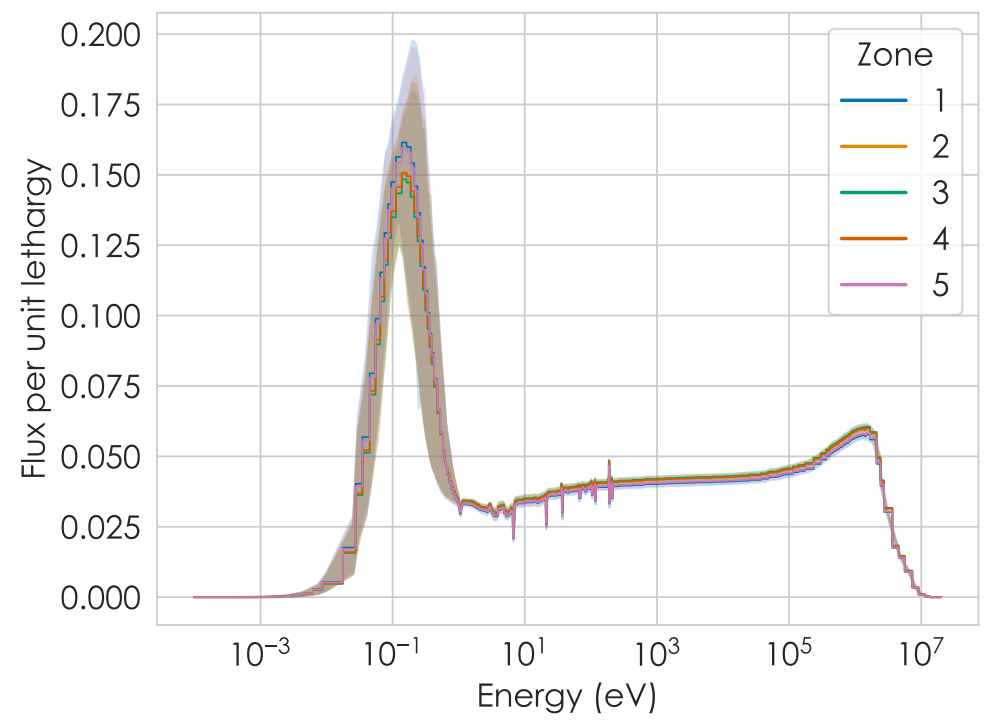

Figure 6. Lethargy-weighted neutron energy spectrum by radial zone (numbered innermost to outermost) for equilibrium-composition core, evaluated at each of the 22 axial material zones. Solid lines represent the average group flux within the radial zone (over all axial zones), with shaded region indicating the standard deviation of the intra-zone flux distribution.

Figure 6 illustrates the radial dependence of the normalized, lethargy-weighted neutron spectrum over the axial length of the core for each of the five radial material zones (numbered in increasing order from the core interior to exterior). Within Figure 6, the multi-group flux is evaluated for each of the 22 axial material regions for each of the 5 radial zones; the standard deviation of the distribution of groupwise fluxes is illustrated as the shaded regions.

Two trends are evident in the flux profile: (1) the flux shape is relatively invariant axially within the core, and (2) the variation in the flux shape primarily occurs near the periphery of the annular core region (i.e., the inner and outermost radial regions). Specifically, the regions closest to the graphite reflectors (region 1 being the closest to the central reflector and region 5 being closest to the outer reflector) exhibit a noticeably higher thermal flux relative to the more central radial locations. Thus, one can plausibly identify two distinct flux "zones" radially within the core, which may alter the effective isotopic transition balance (i.e., higher probability of resonance absorption). This effect will be explored further with respect to the development of one-group ORIGEN cross-section libraries.

\subsubsection{SPATIAL DIFFERENCES IN NEUTRON SPECTRUM CHARACTERISTICS}

Collapsing the neutron spectra into two broad groups (thermal and fast), the core flux profile for the equilibrium-composition core is presented as Figures 7 and 8 for the fast and thermal groups.

In general, the expected flux distribution is seen in the coarse radial zone groupings, wherein the fast flux peaks within the central radial region of the core (corresponding to the power peak, likewise observed in the fission power distribution in Figure 9), whereas the thermal flux is strongly peaked toward the central reflector region but also exhibits considerable peaking around the outer reflector region adjacent to the core. Notably, the fast flux is lowest at the outer radial edge of the core, mirroring similar power tilting observed in prior studies [2]. Conversely, the thermal flux is relatively tightly grouped by radial region, with moderate peaking observed in the innermost radial zone but in general not indicating large gradients over the core region. The axial fission power profile shown in Figure 9 closely tracks with the shape of the thermal flux 

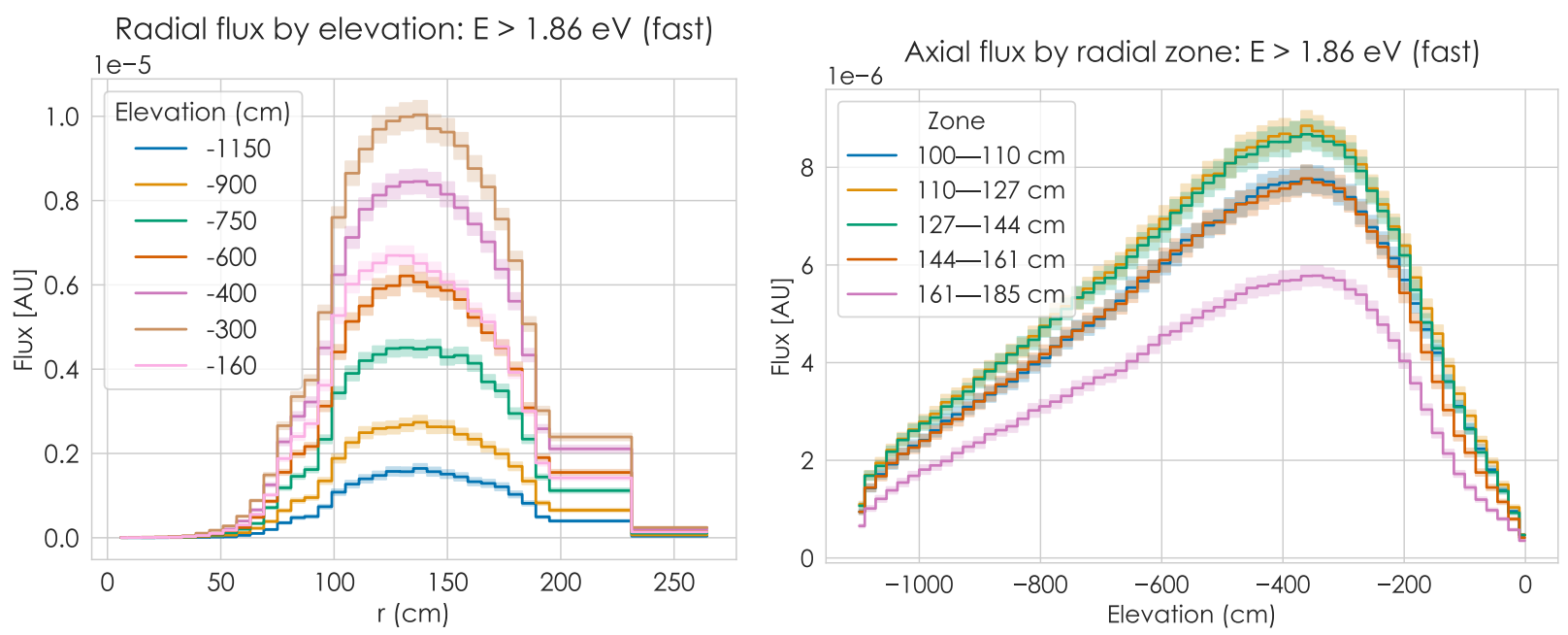

Figure 7. PBMR-400 fast flux $(E>1.86 \mathrm{eV})$ distribution for equilibrium-composition core at $1200 \mathrm{~K}$ isotherm; (left) Radial fast flux by axial elevation $(z=0 \mathrm{~cm}$ corresponds to the top of the active fuel zone); (right) axial fast flux profile by radial zone.
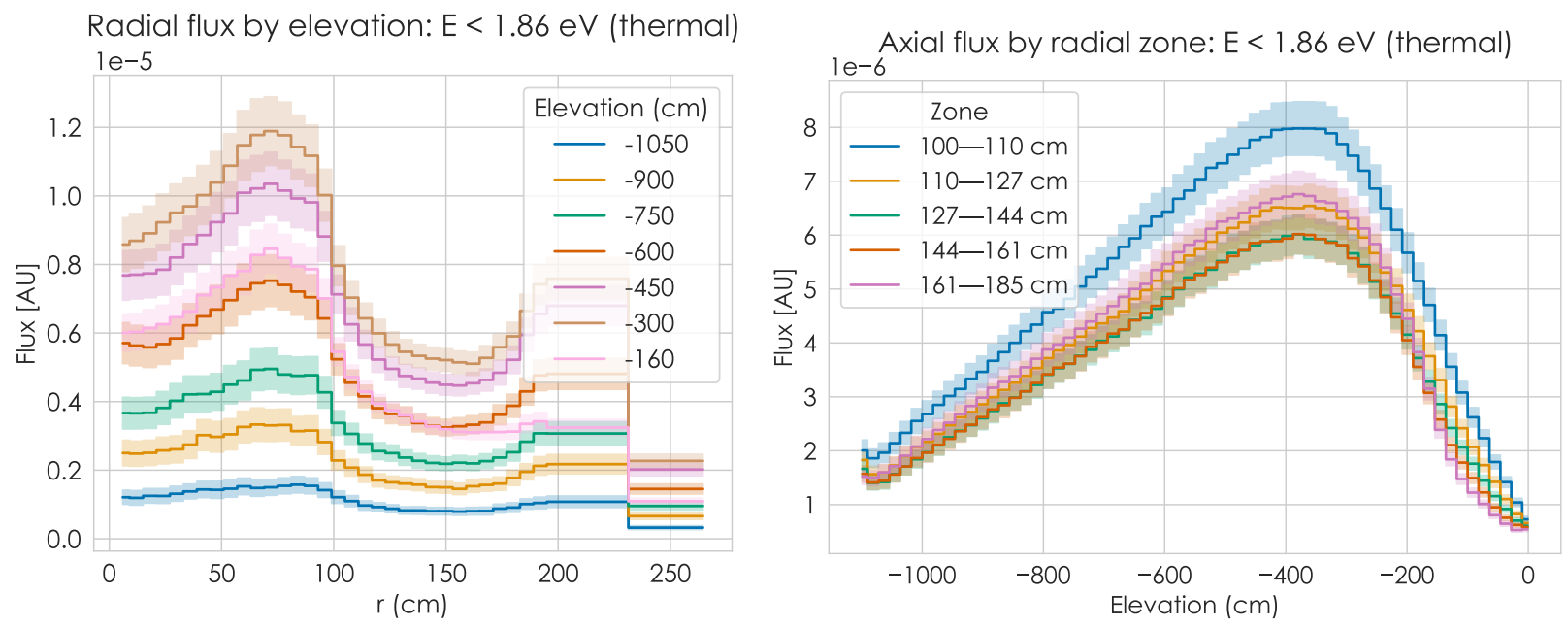

Figure 8. PBMR-400 thermal flux $(E<1.86 \mathrm{eV})$ distribution for equilibrium-composition core at 1200 $\mathrm{K}$ isotherm; (left) Radial thermal flux by axial elevation $(z=0 \mathrm{~cm}$ corresponds to the top of the active fuel zone); (right) axial thermal flux profile by radial zone. 
distribution within the core, consistent with intuitive expectations of the highly-moderated spectrum wherein fissions are primarily driven by thermal neutrons.

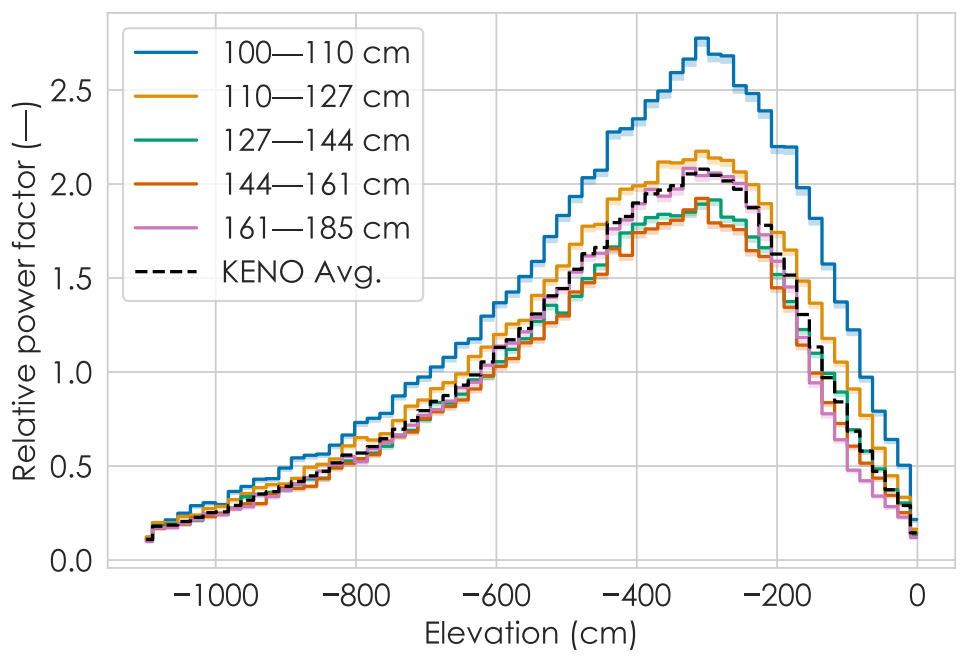

Figure 9. Fission power factor for the PBMR-400 equilibrium-composition core by axial location and radial region. Note that $z=0 \mathrm{~cm}$ indicates the top of the active fuel zone.
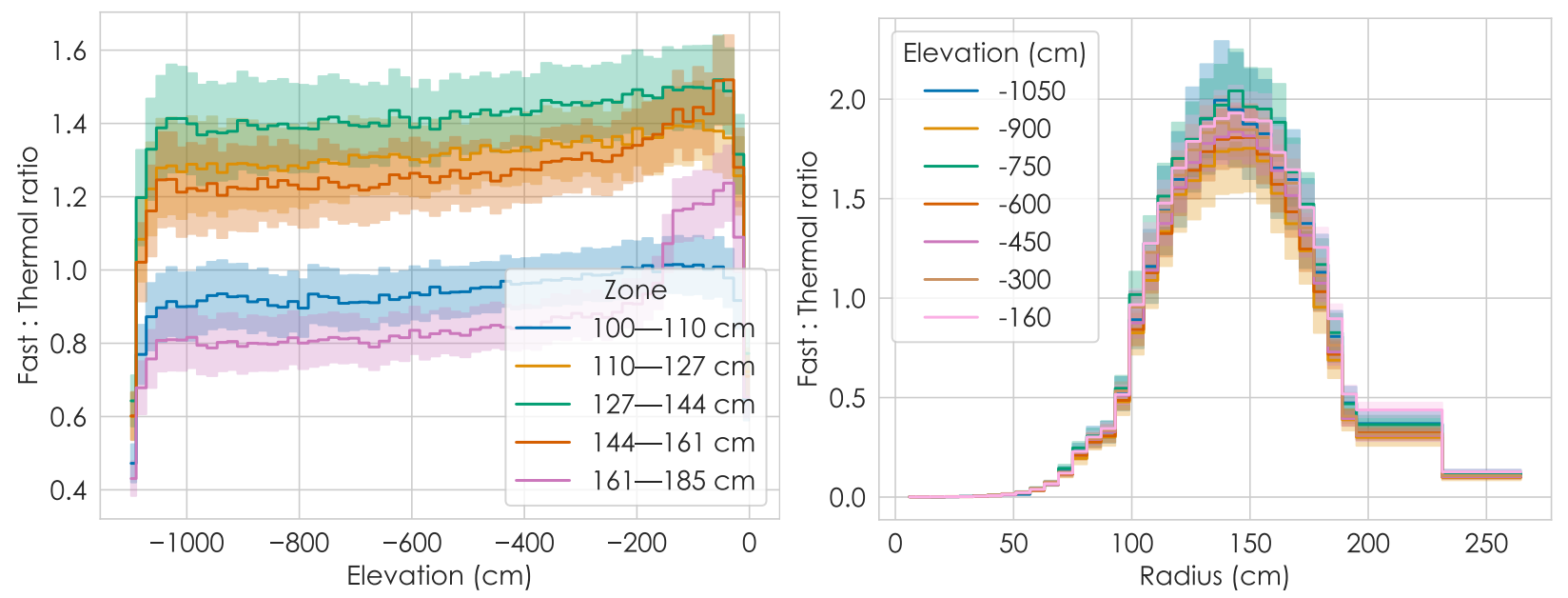

Figure 10. Spectral ratio (fast : thermal flux) for the equilibrium-composition core, along the (left) axial dimension, (right) radial dimension; the thermal-fast boundary is defined at $E=1.86 \mathrm{eV}$. Note that $z=0 \mathrm{~cm}$ indicates the top of the active fuel zone.

Supporting this observation is the ratio of the thermal $(E<1.86 \mathrm{eV})$ to fast $(E>1.86 \mathrm{eV})$ (referred to as the "spectral index") axially over each of the five radial zones, shown in Figure 10. Consistent with the trends seen in Figure 6, the spectral index roughly tracks symmetrically across the radial span of the annular core, wherein the outermost radial regions are significantly more thermalized than the interior zones, implying a logical grouping of 2-3 radial zones for depletion analysis.

Meanwhile, the spectral index remains largely invariant as a function of elevation for any given radial location; this is implied by the relatively flat shape of the spectral index as a function elevation for the five axial zones observed and is confirmed by a subsequent radial analysis of the spectral index at varying axial 
locations.

Taken together, these observations would suggest that treatment of radial zones is sufficient for depletion library development, as it effectively captures the variations in the neutron spectrum throughout the pebble's irradiation history. Variations in the pebble power (i.e., the axial transit behavior) will necessarily influence the discharge isotopic vector of the pebble, because the spectral shape is largely conserved, suggesting minimal changes to the collapsed one-group cross-sections over the history within a given radial zone.

\subsection{CORE PHYSICS PARAMETERS}

This section focuses on the analysis of core physics parameters, such as the temperature coefficient of reactivity and energy of the average lethargy causing fission (EALF); these parameters were evaluated for the full PBMR core for both the fresh core and equilibrium-composition core conditions.

\subsubsection{TEMPERATURE COEFFICIENT OF REACTIVITY}

From the reactivity studies conducted for the full, fresh core (Table 4) and the equilibrium-composition core (Table 5), the temperature coefficient of reactivity $\delta_{k}(T)$ can be estimated using a linear, least-squares fit of the core reactivity $(\rho(T)$, Eq. 3 ) to temperature (Figure 11) for the four system isotherms investigated (see Table 6).

$$
\rho(T)=\frac{k_{\mathrm{eff}}(T)-1}{k_{\mathrm{eff}}(T)}
$$

Table 6. Estimated temperature coefficient of reactivity for PBMR-400 fresh and equilibriumcomposition cores

\begin{tabular}{ll} 
Core & $\alpha_{T}(\mathrm{pcm} / \mathrm{\kappa})$ \\
\hline Fresh & $-9.622 \pm 0.587$ \\
Equilibrium & $-3.342 \pm 0.681$ \\
\hline
\end{tabular}

A substantially more negative temperature reactivity coefficient for the fresh core can be seen, compared with the equilibrium-composition core. One of the primary drivers of this behavior is likely the distribution of fuel in the core: whereas by the time of equilibrium, all of the graphite "dummy" pebbles have been fully swapped out by fuel pebbles (with a higher initial enrichment of $9.6 \%$, compared with the original enrichment of $5.768 \%$ for the fresh fuel pebbles), only about a third of the pebbles in the fresh core are fuel-bearing.

One caveat regarding the derived temperature coefficient is that the value presented here represents a total temperature coefficient, which includes interlinked effects from fuel and moderator temperature feedback. Because the moderator temperature is directly a function of operating power (as well as the helium gas flow rate), the fuel and moderator coefficients were not explicitly decoupled, given that a thermal hydraulic analysis was beyond the scope of this study.

\subsubsection{ENERGY OF THE AVERAGE LETHARGY CAUSING FISSION}

Table 7 summarizes the core-wide EALF for the fresh core and the equilibrium-composition core for the 300, 600,900 , and $1200 \mathrm{~K}$ isotherm models. As expected, the EALF tracks roughly proportional to the system temperature (i.e., nearly all fission in the system is being driven by thermal neutrons). For convenience, the modal neutron energy $(k T)$ for the thermal neutron flux is provided for comparison. 


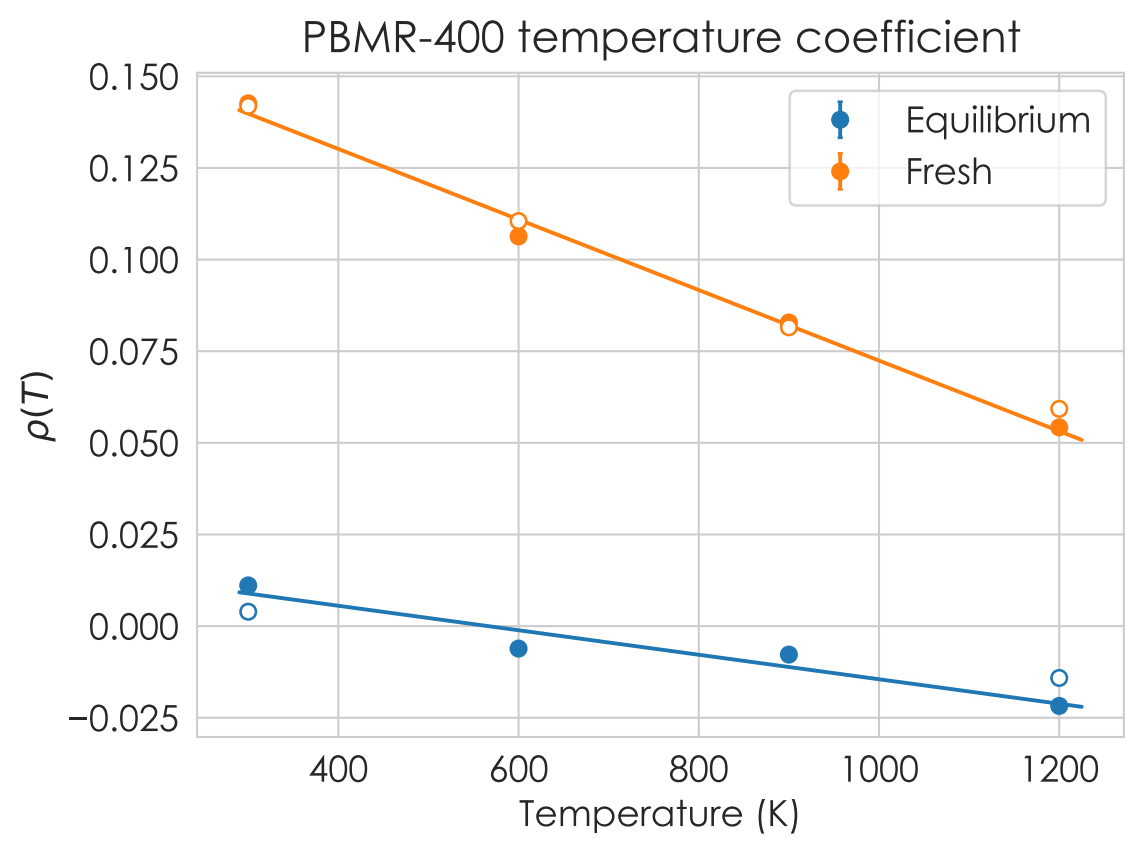

Figure 11. Fitted system reactivity as a function of temperature for isothermal PBMR-400 systems. (Empty circles represent VSOP values from the benchmark problem used for comparison [1])

Table 7. Energy of the average lethargy causing fission $(\mathrm{eV})$ as a function of core temperature.

\begin{tabular}{rl|ll|ll} 
Temperature & $k T$ & \multicolumn{2}{|c|}{ Fresh core } & \multicolumn{2}{|c}{ Equilibrium-composition core } \\
$(\mathrm{K})$ & $(\mathrm{eV})$ & EALF $(\mathrm{eV})$ & $\sigma_{E A L F}$ & EALF $(\mathrm{eV})$ & $\sigma_{E A L F}$ \\
\hline 300 & 0.02585 & 0.03739 & $<10^{-6}$ & 0.054873 & $<10^{-6}$ \\
600 & 0.05170 & 0.06620 & $<10^{-6}$ & 0.098725 & $<10^{-6}$ \\
900 & 0.07756 & 0.09530 & $<10^{-6}$ & 0.14563 & $\sim 10^{-6}$ \\
1200 & 0.1034 & 0.12435 & $<10^{-6}$ & 0.18849 & $\sim 10^{-6}$ \\
\hline
\end{tabular}

As can be seen in Table 7, the system is extremely thermalized, driven predominantly by thermal fission. This observation is consistent with the neutron energy spectra profiles illustrated in Figure 6, where the thermal neutron flux is dominant in the lethargy-weighted space and the fission spectrum peak is greatly diminished. Comparatively, the fresh core shows a roughly $30 \%$ lower EALF, consistent with the additional moderation present in the initial core from the graphite dummy pebbles (which is also observed in the neutron energy profile). 


\section{DEVELOPMENT OF ONE-GROUP ORIGEN CROSS-SECTION LIBRARIES}

Having provided an overview of the full core behavior, we now turn our attention to the depletion behavior of the individual fuel pebble. Here, the goal of this analysis is to identify the minimum set of data required to create ORIGEN reactor data libraries capable of performing rapid depletion calculations with reasonable fidelity compared to a detailed transport-depletion simulation. Factors to be considered include relative shifts in the spectrum as a function of the pebble's irradiation history (i.e., when circulating through different axial and radial zones), effects of the time-dependent power history for depletion (again a function of core-wide parameters such as the flux profile), and local features (e.g., proximity to the control rods).

\subsection{CONSISTENCY OF MODEL SCOPE FOR MULTI-GROUP MONTE CARLO CALCULA- TIONS FOR LIBRARY GENERATION}

One of the goals in establishing a means of developing ORIGEN reactor data libraries for modular pebblebed reactor designs (such as the PBMR) is to determine the minimum viable scope required to faithfully capture the physics of the system. Such a characterization is necessary to make the process of pebble-based library development computationally tractable, given the considerable size of the full-core model. For this study, the full-core model was compared with the infinitely reflected pebble (Figure 2) and a reflected axial slice model (Figure 12). The latter consisted of an axially reflected slice of the system (including the core and outer reflector regions) at the core axial mid-plane. (Note that while Figure 12 illustrates one-quarter symmetry of the axial plane to illustrate detail, the full plane is explicitly modeled). The assumption being tested by the reflected plane model was that radial effects would dominate the neutron spectral characteristics of the core; whereas, given the relatively long neutron mean free path and continuous circulation of fuel material, axial effects were expected to be of secondary importance.

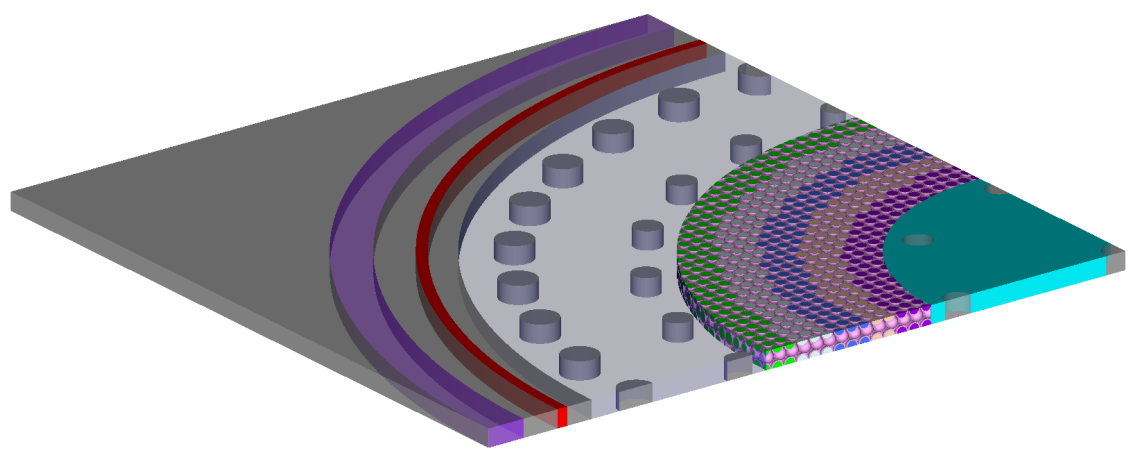

Figure 12. SCALE 6.3 "reflected plane" model, representing an axially-reflected "slice" of the PBMR system at the core axial mid-plane. Rendering is clipped to illustrate detail; the full radial plane is explicitly modeled.

Beyond the most direct assessments of depletion performance (i.e., by looking at the calculated isotopic inventories from a depletion problem using different libraries), a robust assessment of library differences due to the model scope (e.g., reflected pebble, plane, and full core) can be performed by examining the multigroup neutron energy spectrum used for the flux-weighted collapse to one-group reaction cross-sections. Differences in the observed multi-group energy spectrum are thus useful for identifying causes of observed differences in derived one-group reaction cross-sections, as well as for verifying agreement between different models. 


\subsubsection{BURNUP-DEPENDENT EFFECTS ON THE NEUTRON SPECTRUM}

In order to evaluate the relative effects of burnup for the one-group cross-section library development, the multigroup flux spectrum is evaluated as a function of burnup for the infinitely reflected pebble and the reflected axial plane model. The aim of this analysis is to both quantify the relative effect of burnup on onegroup cross-sections (made manifest through shifts in the neutron energy spectrum) as well as to compare the consistency of these effects across different levels of model scope.

Figure 13 illustrates the multi-group neutron flux spectrum for the infinitely-reflected pebble and reflected plane models, both beginning with a fresh fuel configuration. The reflected pebble case shows relatively low change in the neutron spectrum shape with respect to burnup. In marked contrast, the reflected plane with the initial core configuration (i.e., $2 / 3$ dummy pebbles) shows considerably higher shifts in the spectral shape as a function of burnup. Moreover, the spectrum "softens" for the reflected plane case (i.e., the flux distribution shifts to the lower-energy thermal peak monotonically with increasing burnup). The difference is likely explained by the absence of the additional moderator in the reflected pebble case (in the form of the radial reflectors and the graphite dummy pebbles).

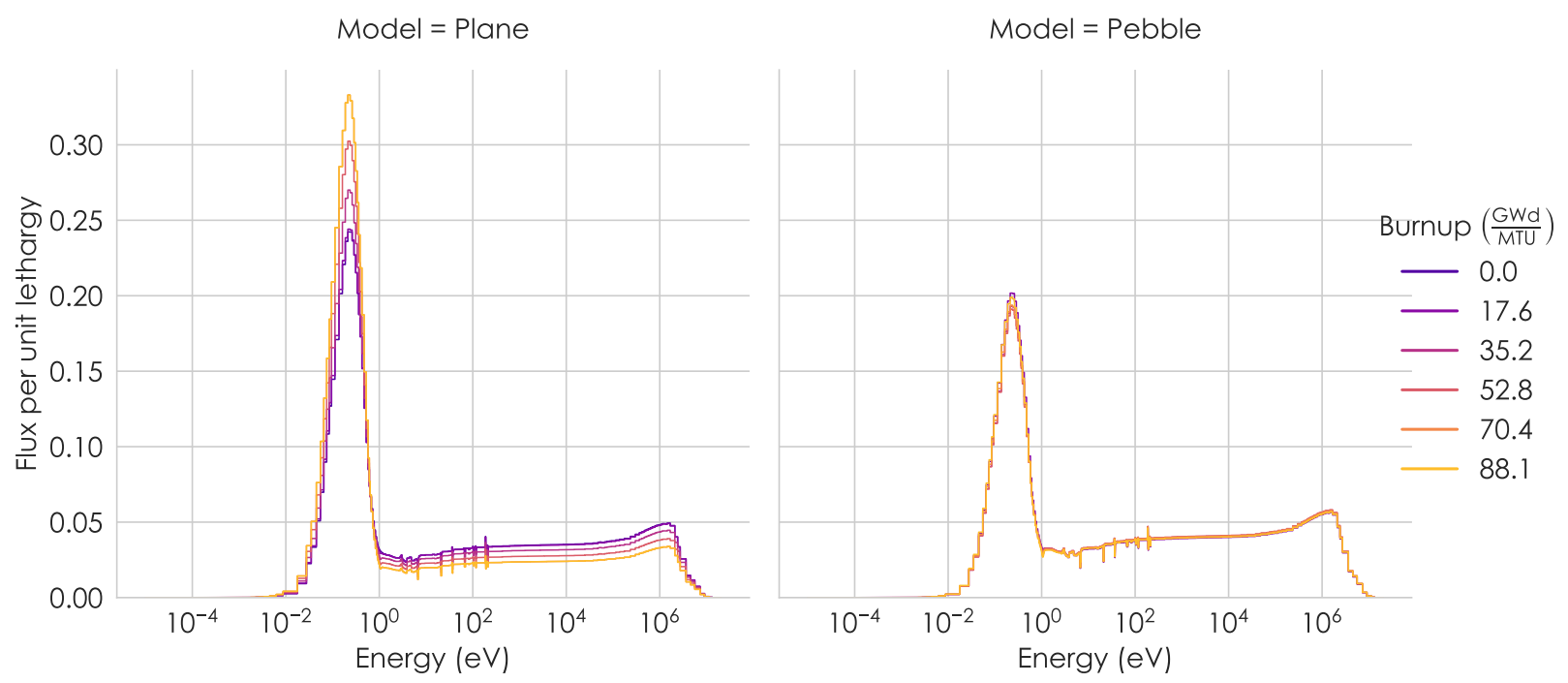

Figure 13. The 252-group collapsing spectrum as a function of burnup for the single, infinitely reflected pebble and a reflected axial plane (core mid-plane), starting from a fresh pebble / plane configuration. The reflected axial plane model includes $2 / 3$ graphite pebbles, significantly influencing the spectral shape. Note that both models show a burnup-dependent spectral shift, but the effect is more pronounced for the plane.

A useful point of comparison of the burnup effect for the PBMR core is the equilibrium-composition core. Because it is assumed that pebbles continuously transit through the core and (after a number of passes) are replaced, the stratified layers can be seen effectively as a static "snapshot" of burnup as a function of axial height. In other words, because the core is at equilibrium, we can therefore assume that the isotopic evolution over the axial length is invariant; thus, the axial height serves as a useful proxy for region-averaged burnup. The equilibrium-composition core inventories provided within the benchmark in reality represent an admixture of pebbles at various burnups (representing a variable number of passes through the core). Because the core is assumed to be at equilibrium, this composition can thus be thought of as representing a relatively invariant regional-average composition surrounding a given pebble of interest; i.e., in any given pass through the core, the neighboring pebble environment is expected to be relatively unchanging at equilibrium within a given radial zone. Thus, depleting a single fresh pebble surrounded by non-depleting 
neighbors (at their respective equilibrium compositions) can be useful for understanding the local evolution of the flux as a function of burnup. This is illustrated in Figure 14, which compares the 252-group collapsing spectrum for each of the 5 radial regions for the equilibrium-composition core at the top and bottom axial regions (thus representing the endpoints of the average burnup within the core).

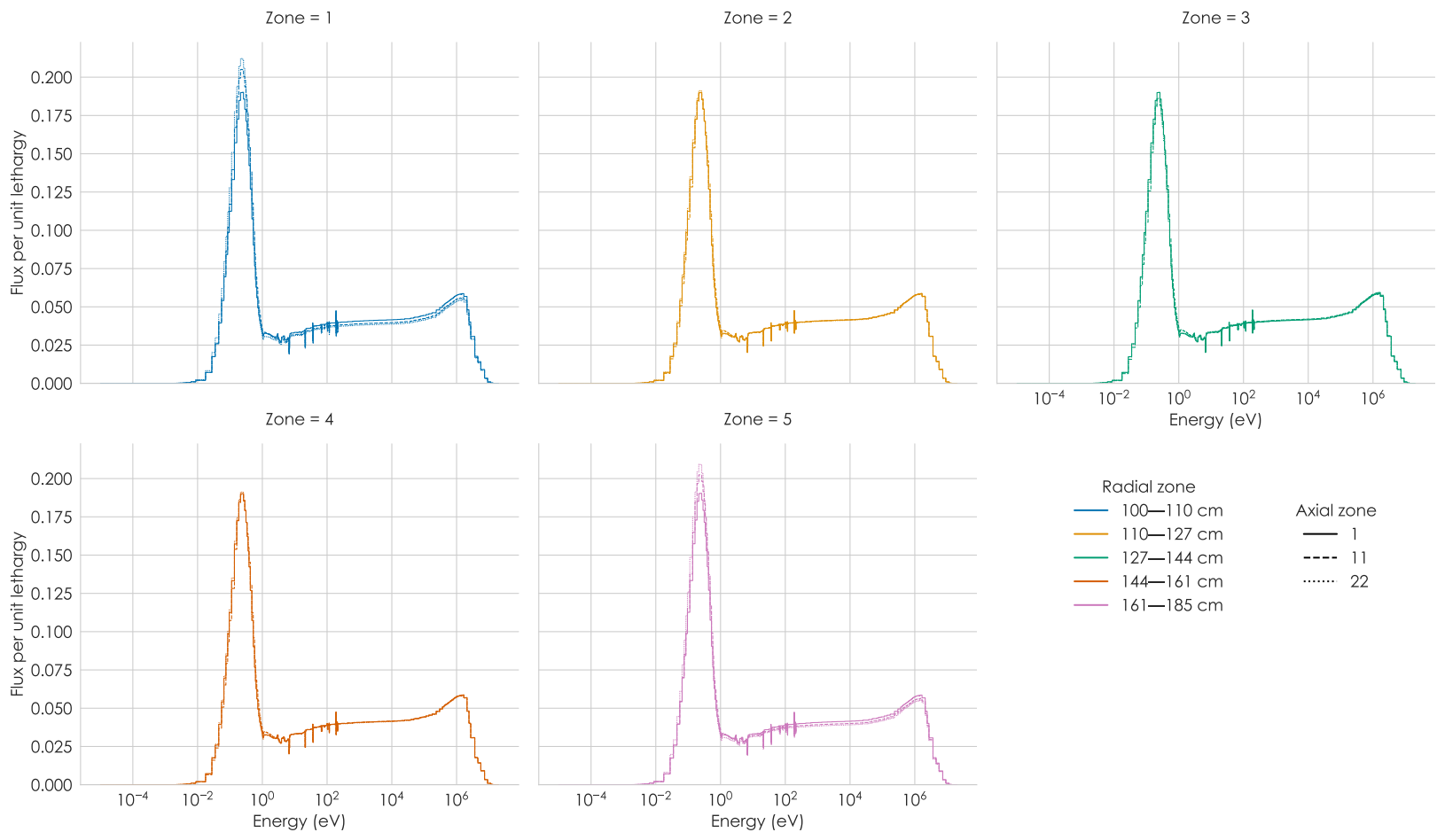

Figure 14. The 252-group collapsing spectrum as a function of radial location for the reflected plane model (equilibrium fuel composition, $1200 \mathrm{~K}$ system temperature), at the top, middle, and bottom axial regions (locations 1,11 and 22, respectively).

Noteworthy in Figure 14 is the relative absence of a trend in burnup with respect to the collapsing flux (here using axial zone as a proxy for burnup); in fact, it is only the outermost radial regions (regions 1 and 5, those nearest to the reflectors) that manifest any discernible differences in the spectral shape as a function of burnup. In Figure 8, the thermal flux is likewise more strongly peaked in these radial zones; likewise, in Figure 10, the spectrum is notably more thermalized in these two radial regions throughout most of the axial length of the core.

A notable feature of the models of the equilibrium-composition core (including the infinitely-reflected pebble and reflected axial plane) was the relatively high degree of agreement between models in terms of the multi-group collapsing spectrum, including between the pebble-level and plane-level models. An example of this behavior can be observed in Figure 15, wherein the collapsing flux is virtually indistinguishable between the full-core and reflected plane models for each of the five radial zones at the targeted axial region of interest (axial region 11, at the core mid-plane). Small but noticeable differences can be seen in the relative thermal peaking factor for the outermost radial regions (i.e., regions 1 and 5, bordering the reflector regions). Likewise notable is the close agreement between the infinitely reflected pebble (equilibrium composition) and the inner radial regions (regions 2-4); i.e., based on the flux shape, the reflected pebble model serves as a good proxy model for the larger system as a whole. This is a significant finding, as it would imply that reasonably accurate pebble depletion libraries could be developed for pebbles within the reactor interior based on relatively simple pebble models with low computational cost. 


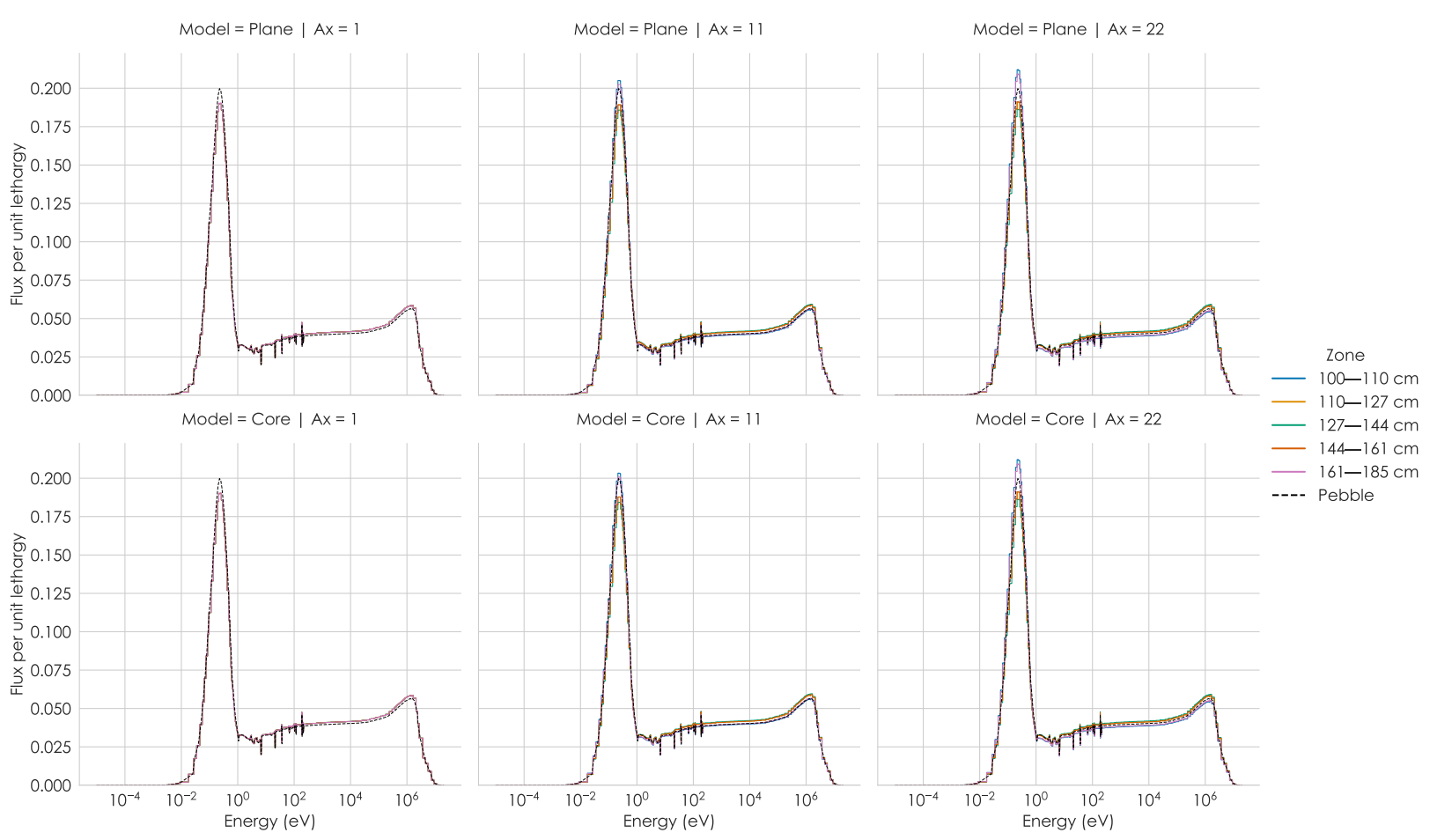

Figure 15. The 252-group collapsing spectrum as a function of radial location for the reflected plane model, full-core model, and the infinitely-reflected pebble, based on equilibrium fuel compositions and a system isotherm of $1200 \mathrm{~K}$.

A useful point of comparison with the equilibrium composition models for the core, plane, and pebble is the relative flux shape profile for the fresh core; Figure 15 shows an overlay of the fuel region for a reflected plane of the starting core. A nominally higher thermal peak relative to the epithermal and fast flux can be seen, with the thermal peak centered at about the same energy as the equilibrium-composition core composition models. This appears to be a consistent feature throughout the core depletion, as is evident from Figure 13, wherein the overall spectrum shape and thermal peak location are unchanged as a function of burnup, with the primary evolution occurring as the balance between the thermal and fast fluxes.

Extending the analysis while using the axial location as a function of burnup, the impact of spectral behavior can be most concisely understood by looking at the collapsed one-group removal cross-sections (i.e., total disappearance, including radiative capture and fission) for the major actinides. These are presented for each of the five radial locations for the reflected plane and full core models (at the top, middle, and bottom of the core for the reflected plane and for all axial regions for the full-core model), given as Figure 16. Noteworthy here is the relative stability of cross-sections such as the ${ }^{240} \mathrm{Pu}$ removal cross-section, which in traditional LWR libraries varies significantly at low burnup that it is typically used to determine library interpolation spacing [16]. Because of the continuous admixture of pebbles at varying burnups in this case, the same "burn-in" gradient does not appear to be present as a function of burnup when the average spectrum within a given fuel region is considered. Moreover, per Figure 16 the variation in one-group cross-sections for the actinides is quite small over the axial domain (indicated by the errors bars, indicating the standard deviation of the loss cross-section across the axial domain), especially when compared with the radial domain. This would appear to indicate the relatively small effect of burnup on the effective one-group cross-sections (assuming axial location as a proxy for average burnup).

The relatively high degree of consistency for the one-group removal cross-sections can be seen as a function 


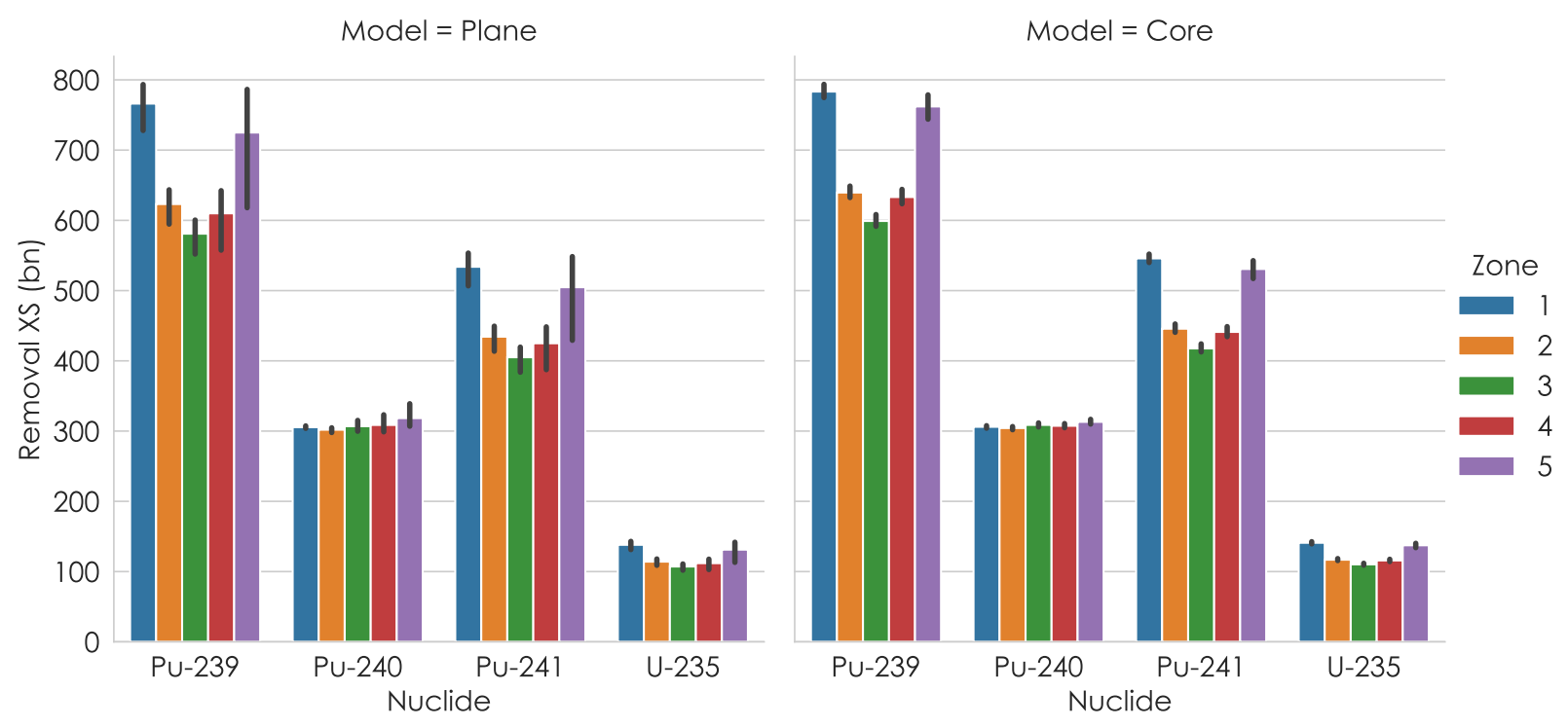

Figure 16. Collapsed one-group removal cross-sections for major actinides for the PBMR-400 equilibrium-composition core for varying axial positions in the (left) reflected plane model, for three separate axial regions (top, middle, bottom) and (right) full-core model (22 axial regions); all evaluations conducted at $1200 \mathrm{~K}$ isotherm.

of radial position between the full-core model and the reflected plane model. The variation in the effective removal cross-sections is relatively small with respect to axial position (on the order of tens of barns or less), indicating that the reflected plane model in fact serves as a useful, reduced-order proxy for capturing the spectrum of the full-core model for purposes of depletion library generation.

By contrast, one-group removal cross-sections in the single infinitely reflected pebble model (Figure 17) exhibit substantial sensitivity to burnup (especially for ${ }^{239} \mathrm{Pu}$ ) as well as temperature. While this model was similar to the larger-scale models (e.g., the reflected plane) in that the system isotherm temperature appeared to be the dominant driver of differences in the one-group removal cross-section behavior, this specific scope exhibited much higher sensitivity to burnup as well as a noticeable interaction term between burnup and the system isotherm temperature (seen in Figure 17). This was very much unlike the larger-scale models, which manifested neither of these features; for the infinitely-reflected pebble, the one-group cross-section behavior more closely resembled that of a traditional LWR assembly. A likely explanation for this result is the effective system represented by an infinitely reflected pebble, in that this model effectively treats the entire system as depleting in concert; i.e., the assumption of a reflected pebble assumes thus that the state of the neighboring pebbles continues to mirror the state of the pebble being depleted-a scenario physically consistent with the LWR modeling approach. Such an approach thus does not (and cannot) account for the "recharge" of fresh material from the continuous circulation of pebbles in the core, which in turn will produce a bias in the observed neutron energy spectrum with burnup. Therefore, the reflected pebble model is likely inadequate to fully capture the depletion behavior inherent to the continuously circulating pebble system.

\subsubsection{DIFFERENCES IN THE NEUTRON SPECTRA ARISING FROM RADIAL POSITION}

The assumption of the burnup gradient being implicitly captured in the axial location is itself limiting is that it captures only the average behavior within a fuel zone and thus overall represented a very limited range of burnups; i.e., it effectively represented the average burnup of each fuel zone over the axial length of the core. In effect, an entire "cohort" of pebbles was being depleted together, thus representing only a small effective 


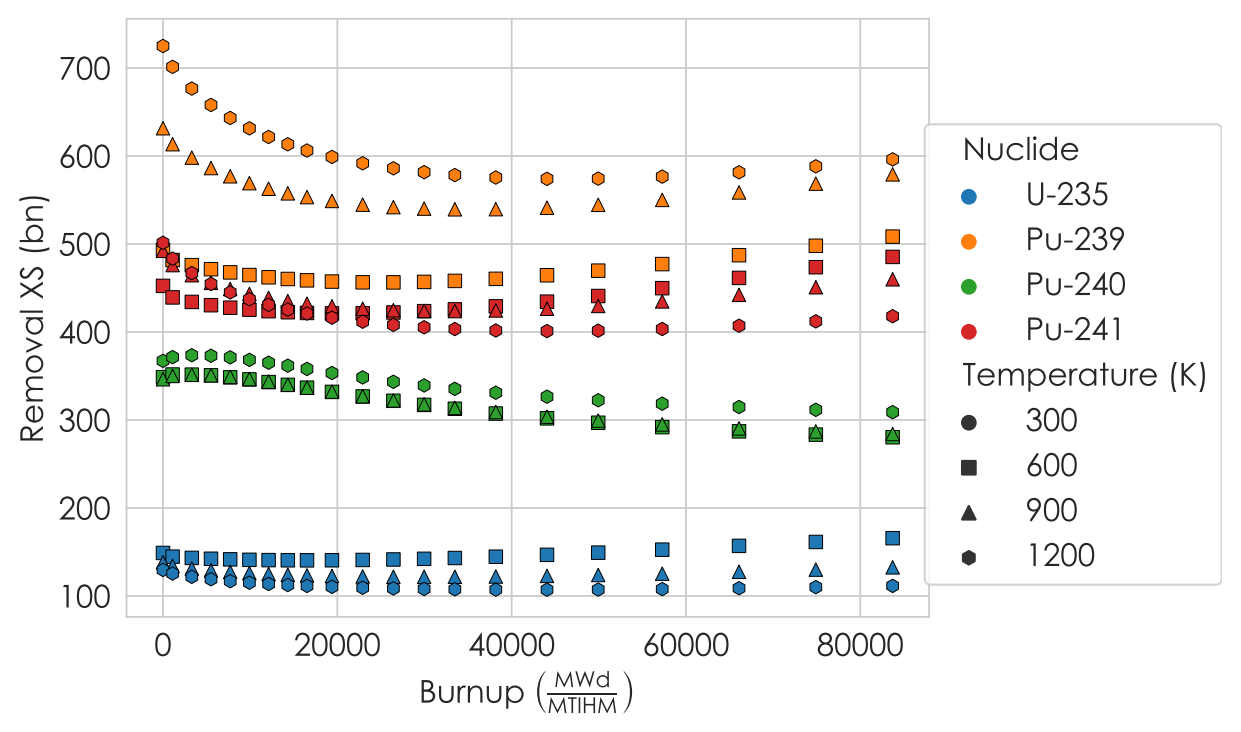

Figure 17. Collapsed one-group removal cross-sections for major actinides for the the infinitely reflected pebble (equilibrium-composition core) as a function of temperature and burnup.

burnup range. This was an inevitable consequence of the nature of the equilibrium-composition core model, in which a series of region-average compositions were specified (representing pebbles at various burnups) without an obvious means of deconvolving their contributions back to discrete pebbles. Making a relatively naive assumption of an equal number of fresh, once-burned, twice-burned, thrice-burned, and four-times burned pebbles and average per-pass burnup of about $17.6 \frac{\mathrm{GWd}}{\mathrm{MTIHM}}$ gave a region-average burnup of 35-52 $\frac{\mathrm{GWd}}{\mathrm{MTIHM}}$ as the fuel passed through the core. For LWR assemblies, the removal coefficients of major actinides exhibit relatively little change over this burnup interval.

An alternative approach would be to evaluate the collapsing spectrum for a fresh pebble over the entire burnup range while surrounding it with an invariant (non-depleting) neighbor material, thus effectively simulating the continuous "churn" of neighbor pebbles around an individual pebble and enabling a more accurate examination of burnup behavior at the individual pebble level. Specifically, given the continuous feed and removal of fuel pebbles, the core would approach an equilibrium admixture of pebbles of different burnups (reflected as the equilibrium-composition core composition). Therefore, for any given batch of fresh pebbles entering the core, the surrounding cohort of pebbles would be relatively invariant. (This approach is thus in contrast to the depletion of the infinitely reflected pebble, wherein effectively the pebble is surrounded by pebbles at identical burnup at all times.)

One means of estimating the most pronounced location effects for a fresh pebble within the equilibriumcomposition core would be to deplete one fresh pebble surrounded by a representative set of pebbles (at the equilibrium composition) in which only the fresh pebble is depleted (i.e., the composition of the surrounding pebbles is kept fixed). This approach would only approximate differences in the overall spectral effects throughout the burnup history by assuming that the pebble would remain in a single radial position throughout its depletion history; but it could nonetheless be useful for elucidating burnup-dependent differences in the spectrum as a function of the radial position. This approach is demonstrated in Figure 18 which illustrates the neutron energy spectrum for the single fresh pebble depleted to a burnup of approximately $90 \frac{\mathrm{GWd}}{\mathrm{MTIHM}}$ at different radial locations within the reflected (static) plane. As can be seen, the separation in the neutron spectra remains quite prominent over the pebble burnup toward the radial boundaries (zones 1 and 5 ), in which the spectrum is noticeably softer. In other words, the observed spatial differences in spectrum 
as a function of radial position appear to persist even at very high burnups, indicating that the radial position effect is a first-order spectral effect that does not diminish with increasing burnup.

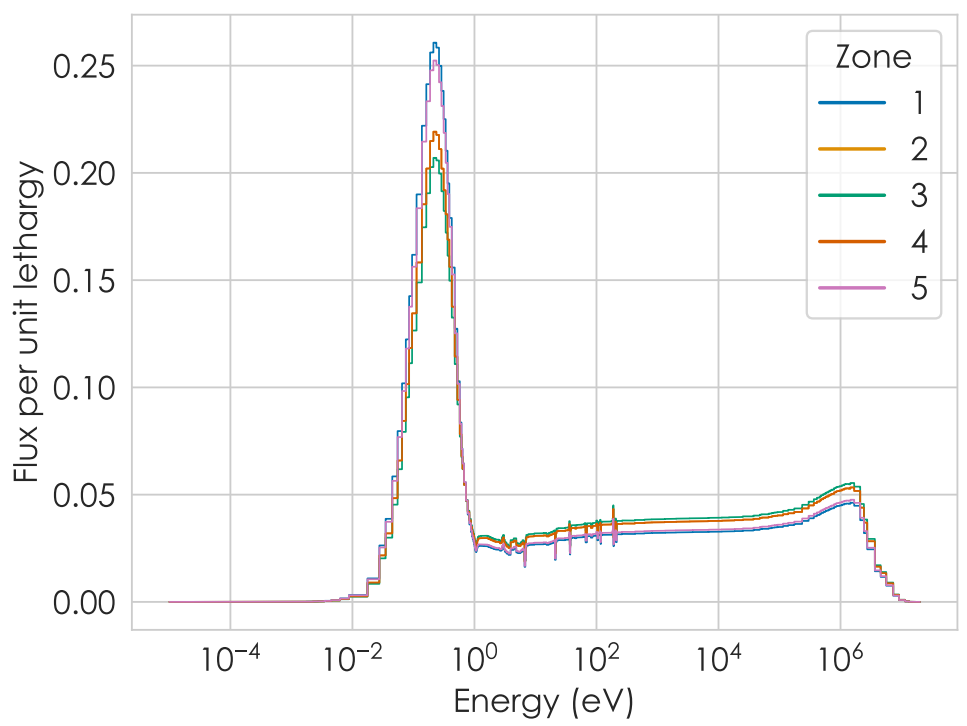

Figure 18. 252-group collapsing spectrum as a function of radial location for a single (fresh) pebble depleted within a static radial plane of pebbles at equilibrium composition (referred to as the "marinade" model), with the depleted pebble at approximately $90 \frac{\mathrm{GWd}}{\mathrm{MTIHM}}$ burnup and a system temperature of $1200 \mathrm{~K}$.

Further illustrating this analysis is Figure 19, which shows the impact of radial position over burnup for the one-group removal cross-sections of major actinides in the collapsed ORIGEN reactor library. Here, the separation in the one-group removal cross-sections by radial zone is especially prominent, especially for the fissile plutonium isotopes $\left({ }^{239} \mathrm{Pu}\right.$ and $\left.{ }^{241} \mathrm{Pu}\right)$. Conversely, the effect of burnup is relatively subdued, especially for traditionally more burnup-sensitive nuclides like ${ }^{240} \mathrm{Pu}$. This finding indicates that in this particular scenario (wherein the pebble is surrounded by a constant neighbor population, in effect approximating the "churn" of continuous circulation), burnup is a secondary driver of changes in the neutron spectrum compared with the radial position. This behavior is largely consistent with the behavior of the multi-group axial flux shape (Figure 14) and the one-group cross-sections (Figure 16). For each of these cases, taking the axial position as a rough proxy for burnup, again the radial location is the predominant factor in determining the effective removal cross-section.

For practical modeling applications, the pebble would most likely be distributed across different radial zones throughout its burnup history, thus "homogenizing" spectral effects on the discharge isotopic vector. That is, the variation in radial locations over the pebble's history would likely lead to an averaging of differences owing to different radial locations over the burnup history. Thus it would lead to lower variance in the depleted isotopic compositions of pebbles compared with the case where they were exclusively burned in a single radial location. Nonetheless, for applications involving short time intervals following irradiation (such as for accident source term analysis), it is likely that the most recent irradiation spectrum would influence the distribution of short-lived fission products (and thus key properties such as decay heat) and thus separate treatment of the radial position for depletion library development appears to be warranted. This issue will be further evaluated for its net impacts on the macroscopic properties (i.e., from decay heat) in evaluating the performance of the one-group libraries for depletion in the following section. 


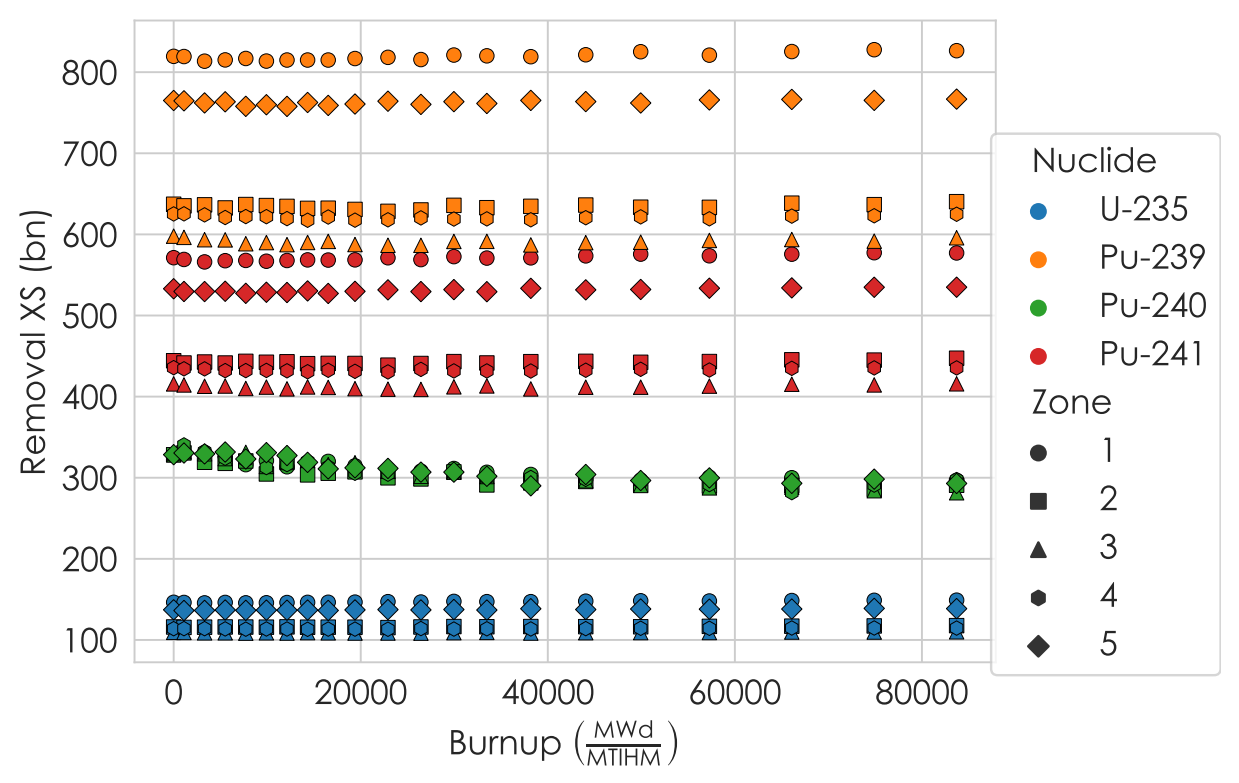

Figure 19. One-group removal cross-section for major actinides as a function of radial location and burnup for a single (fresh) pebble depleted to approximately $90 \frac{\mathrm{GWd}}{\mathrm{MTIHM}}$ within a static radial plane of pebbles at equilibrium composition with a system temperature of $1200 \mathrm{~K}$.

\subsubsection{COMPARISON TO HISTORIC 1-D APPROACHES}

The approach outlined here bears some similarity to a long-prior attempts to produce an Origen reactor data library for the PBMR-400. Specifically, Stoker et. al. considered the problem from the perspective that the relatively high spacing between fuel micro-kernels would yield a scattering mean free path of $2.6 \mathrm{~cm}$, with an absorption mean free path of $31 \mathrm{~m}$, indicating that the incident neutron spectrum for a given pebble can be influenced by pebbles far from the pebble being considered [23]. One of the chief insights of this work then is to posit that the flux spectrum within a given fuel sphere is determined primarily by the average spectrum of the surrounding fuel pebbles [23].

To test this theory, Stoker et. al. created a 1-D spherical reactor model consisting of a single fuel sphere at the center surrounded by a $1 \mathrm{~m}$ driver zone (composed of fuel at the core-average burnup of $45 \frac{\mathrm{GWd}}{\mathrm{MTIHM}}$ ) and a $1 \mathrm{~m}$ graphite "reflector". Comparing the one-group cross-sections for the "fuel" sphere at a nominal burnup of 0 and $80 \frac{\mathrm{GWd}}{\mathrm{MTIHM}}$ to a reference initial burnup of $45 \frac{\mathrm{GWd}}{\mathrm{MTIHM}}$ revealed only very small differences for most major species (generally less than 2\%). By contrast, varying the "driver" region composition to 0 and 80 $\frac{\text { GWd }}{\text { MTIHM }}$, respectively (with a nominal "fuel" region burnup of $45 \frac{\mathrm{GWd}}{\mathrm{MTIHM}}$ ) produced substantial differences in calculated nuclide inventories, particularly for species such as ${ }^{110 m} \mathrm{Ag}(-21 \%,+11 \%),{ }^{134} \mathrm{Cs}(+6.3 \%,-12 \%)$, ${ }^{239} \mathrm{Pu}(+4.4 \%,-10 \%)$, and ${ }^{241} \mathrm{Pu}(+6.9 \%,-13.1 \%)$

\subsection{EFFECTS OF POWER HISTORY ON ONE-GROUP CROSS-SECTIONS}

For this analysis, the effects of the power history on the one-group cross-section library will be quantified for a single-pebble model (infinitely reflected), assuming two scenarios: first, a uniform depletion at the reactor average power, and second a non-uniform depletion history taking into account the axial flux profile, with the working assumption that the specific pebble power at a given axial zone is directly proportional to the thermal flux (shown as Figure 20). A detailed list of assumptions used for this analysis exercise is outlined as Table 8. For this analysis, the primary time domain of interest will be from discharge to up to 10 days post-discharge, spanning the range of primary interest for severe accident source term analysis. It 
is likewise here that the fission products most sensitive to power history (i.e., those whose activities rapidly reach saturation) are expected to show the greatest prominence.

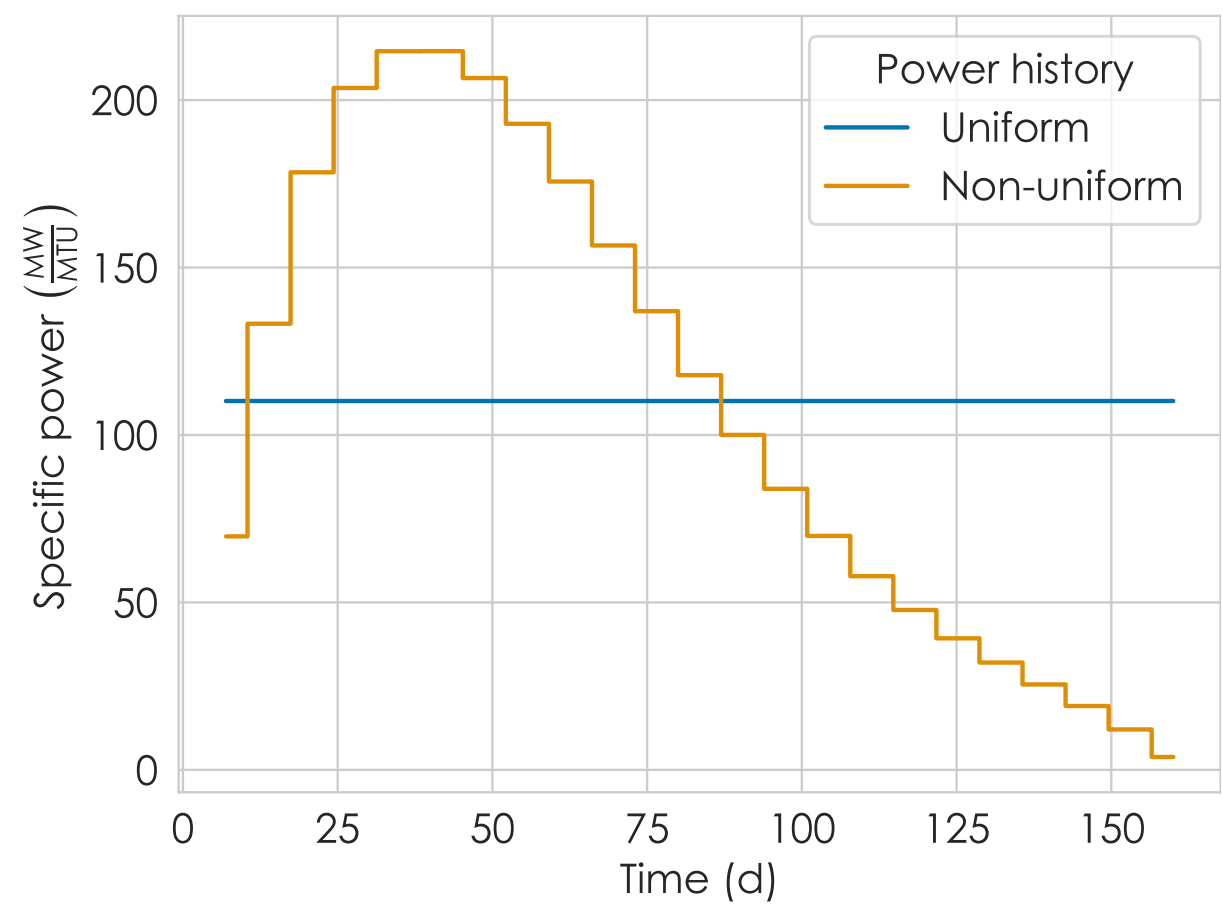

Figure 20. Comparative power histories used representing one transit of a pebble through the core

Table 8. Pebble dimensions and modeling assumptions used for characterization of non-uniform power history on discharge isotopic composition.

\begin{tabular}{ll} 
Fuel region radius $(\mathrm{cm})$ & 2.5 \\
Fuel-free graphite layer thickness $(\mathrm{cm})$ & 0.5 \\
Total pebble radius $(\mathrm{cm})$ & 3.0 \\
Lattice type & Dodecahedral / FCC \\
Effective pebble-to-pebble pitch $(\mathrm{cm})$ & 6.4 \\
Initial enrichment $\left(\%{ }^{235} \mathrm{U}\right)$ & 5.768 \\
Nominal average power $(\mathrm{W} / \mathrm{kg})$ & 110.143 \\
Irradiation time $(\mathrm{d})$ & 160 \\
\hline
\end{tabular}

In general, the effect of the power history on the collapsed one-group cross-sections is negligible, especially compared with larger effects such as the system temperature, as is seen in Figure 21.

\subsection{TEMPERATURE EFFECTS ON ONE-GROUP CROSS-SECTION LIBRARIES}

The effects of system temperature (particularly for the graphite reflectors) are well known to be of significant consequence for graphite-moderated systems like the PBMR. In the case of the PBMR, this feature served as an explicit design safety feature, in that temperature served as a strong negative reactivity feedback (as discussed previously in Section 3.2.1). In terms of the neutron spectrum, an increasing system temperature ultimately led to a shift in the thermal peak of the neutron spectrum, both effectively resulting in a "hardening" of the spectrum as well as shifting the neutron flux much closer to important absorption resonances 

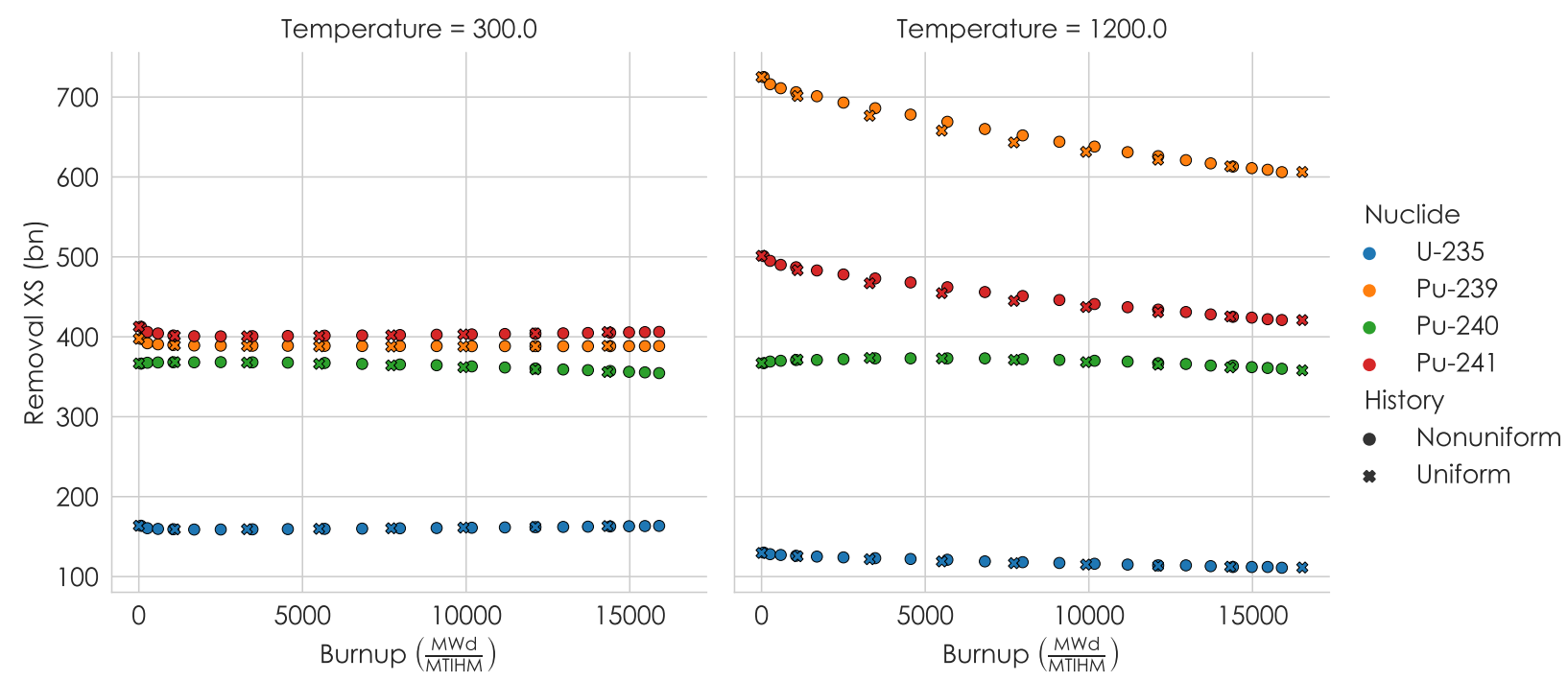

Figure 21. Effective one-group removal cross-sections for the single reflected (fresh) pebble, irradiated at uniform and non-uniform power histories over one core transit (approximately $17.6 \frac{\mathrm{GWd}}{\mathrm{MTIHM}}$ ) for (left) $300 \mathrm{~K}$ and (right) $1200 \mathrm{~K}$ system isotherm.

of ${ }^{238} \mathrm{U},{ }^{239} \mathrm{Pu}$, and ${ }^{240} \mathrm{Pu}$. This is illustrated in Figure 22 (which shows the collapsing multi-group flux for the reflected plane model of the fresh core as a function of system [isotherm] temperature) and in Figure 23 (which illustrates the same temperature-driven shift for the equilibrium-composition core). (Note that the effects of Doppler broadening were also evaluated as part of this analysis, however the effects are considerably less visible compared to the spectral shift and thus are omitted for clarity.)

As Figures 22 and 23 illustrate, the shape and location of the thermal peak are strong functions of the fuel and reflector temperature; i.e., this proves to be a far more prominent feature affecting the spectral shape (and thus effective one-group cross-sections) compared with burnup. Hence, consideration of the average fuel temperature would appear to be a first-order consideration for library development of these types of high-temperature systems, which show strong temperature-based feedback.

Examining this effect on the level of collapsed, one-group cross-sections (Figure 24) shows that the effect of the system isotherm temperature both is quite prominent and shows a small positive covariant relationship with the radial position. Specifically, for the fissile plutonium species ( ${ }^{239} \mathrm{Pu}$ and ${ }^{241} \mathrm{Pu}$ ), the difference in the effective one-group removal cross-section in the radial exterior and interior regions grows as a function of temperature. While the effective removal cross-section behavior is roughly linear as a function of temperature for each region, a cursory inspection of Figure 24 indicates that the respective slopes of these functions differ with radial position, indicating that, much like radial location, temperature likewise acts as a first-order parameter for future library interpolation. 


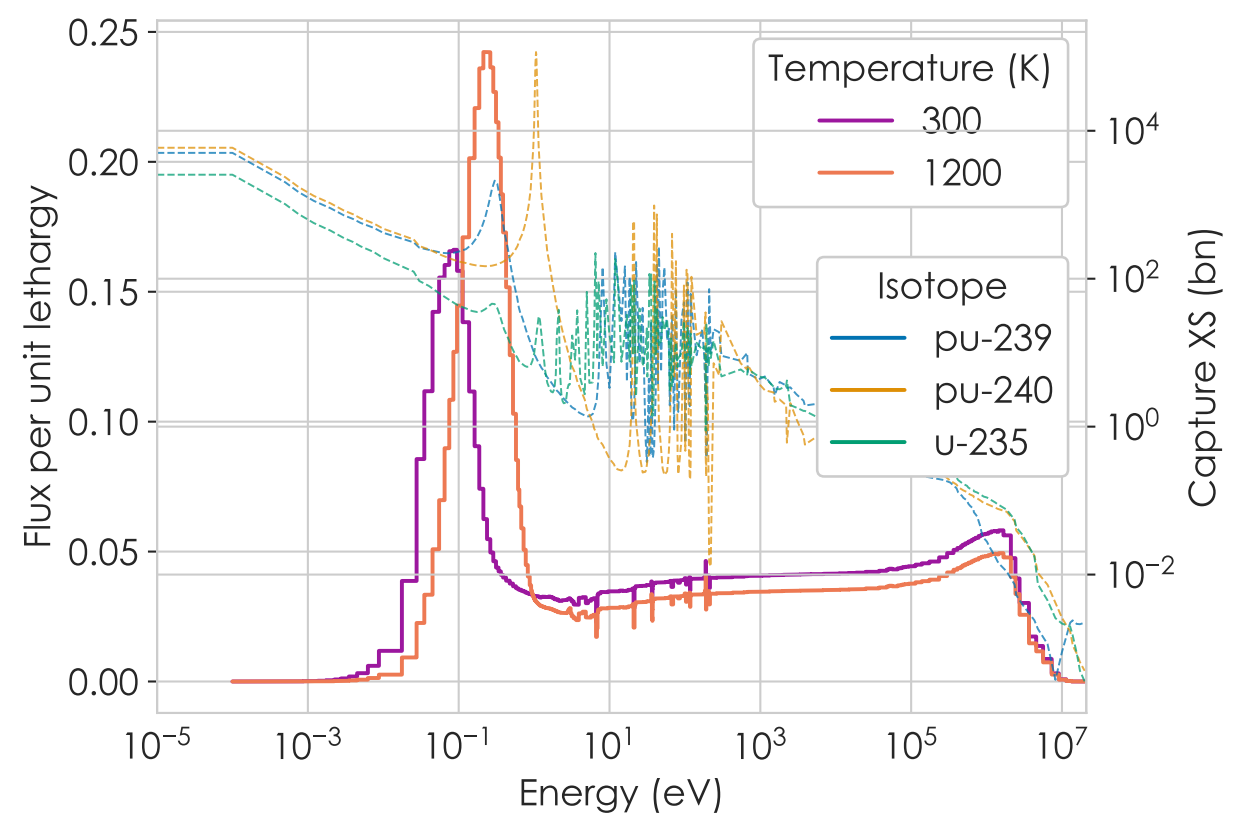

Figure 22. The 252-group collapsing spectrum as a function of temperature (system isotherm) for the fresh core, reflected plane model, with corresponding radiative capture cross-sections for major actinidess overlaid for reference. Note the shift of the thermal neutron distribution onto the ${ }^{239} \mathbf{P u}$ giant resonance with increasing temperature.

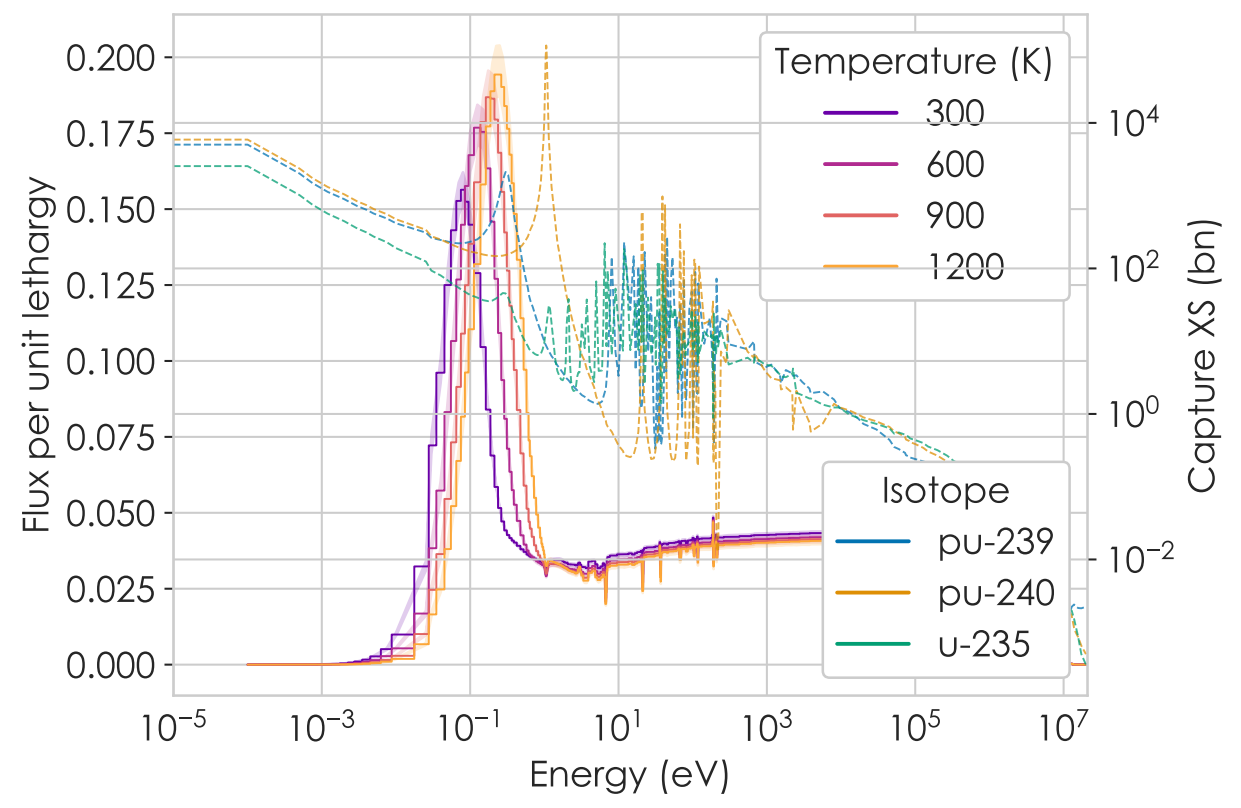

Figure 23. The 252-group collapsing spectrum as a function of temperature (system isotherm) for the equilibrium-composition core, reflected plane model, with corresponding radiative capture crosssections for major actinidess overlaid for reference. Variations in the flux shape by radial zones are represented as shaded areas. 


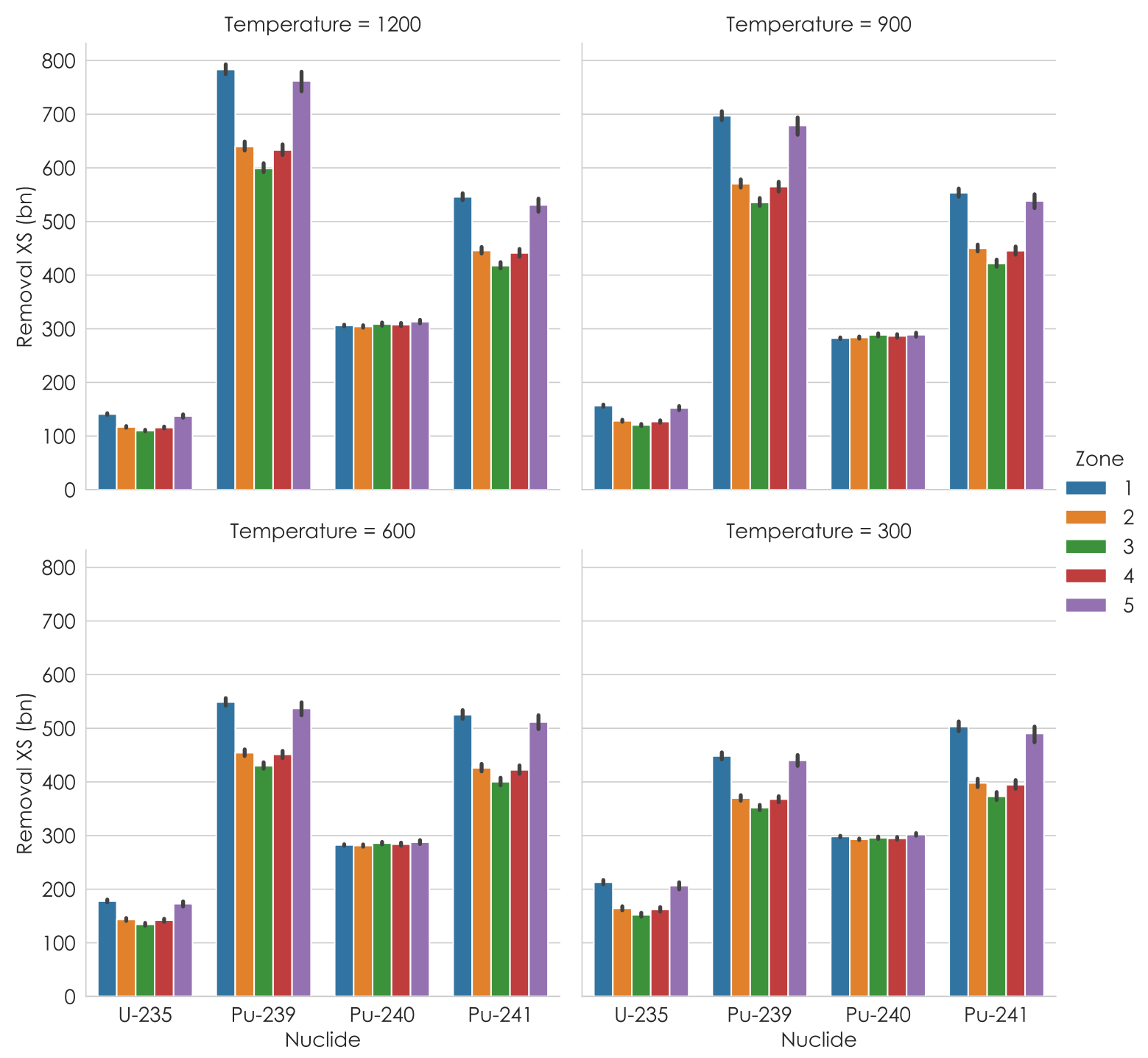

Figure 24. One-group removal cross-section for major actinides as a function of temperature (K) and radial location for the equilibrium-composition core (full core model). Note the divergence in the removal cross-sections for fissile plutonium isotopes ${ }^{239} \mathrm{Pu}$ and ${ }^{241} \mathrm{Pu}$ between the outer radial zones and the interior zones with increasing temperature. 


\subsection{EFFECT OF MODEL SCOPE ON CALCULATED ONE-GROUP CROSS-SECTIONS}

A comparison of effective one-group cross-sections across the full core (Figure 16), the reflected plane (Figures 16, 19, and 24), and the infinitely reflected pebble models (Figure 17) shows the predominant influence of accounting for the radial dimension with respect to capturing effective one-group removal crosssections. According to Figure 16, the reflected plane model yields nearly identical one-group cross-sections for the equilibrium-composition core model for equivalent radial positions. While the relative axial position chosen for modeling the reflected plane introduces a minor amount of variance, this overall effect is small compared to that of the radial location on the one-group cross-sections.

Conversely, modeling the system as an infinitely reflected pebble (Figure 17) exhibits substantial inconsistency with the full-core model; it underestimates the average one-group removal cross-sections for ${ }^{239} \mathrm{Pu}$ and ${ }^{241} \mathrm{Pu}$ by $10 \%$ or more while likewise demonstrating burnup-dependent behavior not observed within the reflected plane model (featuring depletion of a single pebble in a static reflected plane, representing the continuous churn of neighboring material). The difference is clearly attributable to the assumed depletion behavior of the neighboring pebbles; i.e., the reflected pebble model assumes simultaneous depletion of all surrounding material, introducing the observed burnup-dependent behavior.

The net effect of the choice of model scope is likewise observed when the collapsed one-group library is used to perform stand-alone depletion calculations. If the generic assumptions outlined within Table 8 are used to perform a single-pass depletion of a representative pebble, the mass differences at discharge (relative to the full-core model for the central radial region furthest away from the reflectors at core mid-plane) are given in Table 9.

Table 9. Impact of library-generating model on discharge isotopic inventories for a single-transit depletion cycle, expressed as the relative inventory difference compared with depletion using a onegroup library derived from the full-core model. Full core and reflected plane models assume the pebble is at the radially centermost location (farthest from reflectors), core axial mid-plane.

\begin{tabular}{ccc}
\hline \multirow{2}{*}{ Isotope } & \multicolumn{2}{c}{ Relative mass difference to full-core $(\%)$} \\
& Reflected plane & Reflected pebble \\
\hline${ }^{235} \mathrm{U}$ & -0.29 & -0.72 \\
${ }^{239} \mathrm{Pu}$ & -2.53 & -3.77 \\
${ }^{240} \mathrm{Pu}$ & -4.31 & -10.3 \\
${ }^{241} \mathrm{Pu}$ & -0.44 & +2.65 \\
\hline
\end{tabular}

Within Table 9, the mass difference for depletion calculations using an infinitely reflected pebble from the full-core calculation (at the radial center and axial midplane) is roughly double that of the comparative calculation using the reflected plane model in the same corresponding position for each of the major actinides investigated. Notably, the magnitude of the mass difference is most significant for ${ }^{240} \mathrm{Pu}$, although this nuclide exhibits a far smaller difference in the burnup-dependent one-group removal cross-section behavior between the reflected plane model (Figure 19) and the infinite pebble model (Figure 17). This difference is likely attributable instead to the substantial difference in the ${ }^{239} \mathrm{Pu}$ removal cross-section behavior between the two models. The reflected plane model exhibits both an overall flatter response to burnup (with very little change over the entire burnup range) and a lower average removal cross-section (i.e., the reflected pebble model gives a one-group removal cross-section ranging from 0 to $18 \%$ higher than that of the reflected plane model, converging toward the latter with increasing burnup). The ${ }^{240} \mathrm{Pu}$ removal cross-section remains about 40 bn higher for the reflected pebble model (a difference of about 10\%); these trends are illustrated in Figure 25 . 

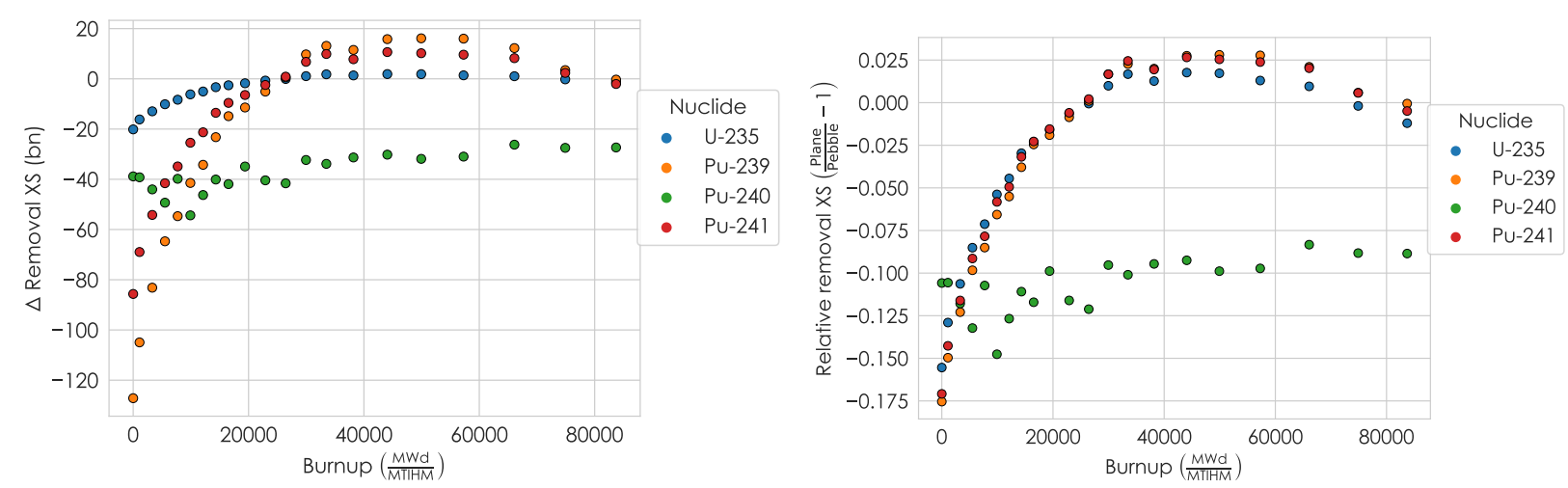

Figure 25. (Left) Absolute and (right) relative difference in calculated one-group removal crosssections for major actinides for the reflected plane and infinite pebble models.

Taking these two observations together thus explains much of the observed difference in ${ }^{239} \mathrm{Pu}$ and ${ }^{240} \mathrm{Pu}$ inventories between the models, as the average one-group removal cross-section for both nuclides was appreciably higher for the earliest burnup period (up to $20 \frac{\mathrm{GWd}}{\mathrm{MTIHM}}$ ), encompassing the domain evaluated here. Thus, the reflected pebble model will overestimate captures by both species to a greater degree than the reflected plane model, with the overestimation of capture by ${ }^{240} \mathrm{Pu}$ being greater and more persistent over the life of the fuel for the pebble-based model.

Looking now to the relative impact of the radial location within the full core and reflected plane models, the impact on the calculated isotopic inventories of major actinides as a function of the relative position within the core (relative to the most central location, farthest from the graphite reflectors) is given for both models in Table 10.

Table 10. Impact of radial library position on isotopic inventories after one pass through the core (a burnup of approximately $17.6 \frac{\mathrm{GWd}}{\mathrm{MTIHM}}$ ), expressed as the relative mass difference (in percentage) compared with depletion using the respective one-group library corresponding to the centermost radial location (radial position \#3).

\begin{tabular}{c|cccc|cccc}
\hline \multirow{3}{*}{ Radial position } & \multicolumn{6}{|c}{ Relative mass difference to central radial position (\%) } \\
& ${ }^{235} \mathrm{U}$ & ${ }^{239} \mathrm{Pu}$ & ${ }^{240} \mathrm{Pu}$ & ${ }^{241} \mathrm{Pu}$ & ${ }^{235} \mathrm{U}$ & ${ }^{239} \mathrm{Pu}$ & ${ }^{240} \mathrm{Pu}$ & ${ }^{241} \mathrm{Pu}$ \\
\hline 1 & -2.85 & -31.3 & -18.7 & -34.3 & -3.14 & -35.4 & -21.0 & -38.1 \\
2 & -0.76 & -9.14 & -4.84 & -10.0 & -0.66 & -8.84 & -3.24 & -11.4 \\
4 & -0.74 & -9.00 & -4.89 & -9.37 & -0.41 & -6.34 & -2.68 & -5.88 \\
5 & -2.77 & -30.6 & -18.6 & -32.6 & -2.53 & -29.6 & -17.1 & -31.1 \\
\hline
\end{tabular}

Here, the effect of moving outward from the central-most radial location is both relatively large and symmetrical in nature. The explanation for this effect is likewise relatively intuitive, deriving from the observable difference in the neutron spectral index as one moves toward the reflector regions (resulting in a substantial softening of the neutron spectrum, an effect observed in the zone-wise one-group cross-sections portrayed in Figure 19).

Notably, the effect of the radial location of the one-group library dominated any effect arising from the use of the full-core model versus a single reflected plane. In other words, the radial location of a given pebble within the core (in terms of its proximity to the reflector regions) is far more prominent in influencing 
one-group cross-section behavior than features such as the relative axial position of the pebble within the core. This in turn justified the treatment of depletion within the PBMR core from a "pebble" level (i.e., calculations of depletion based primarily on the orientation of the pebble within the core over its irradiation cycles) compared with the "core" level (in which the composition of neighboring pebbles is expected to significantly influence the collapsing spectrum used for the depletion of any given pebble).

When the inventories of major actinides following one irradiation cycle were compared for comparable radial positions, in general, the differences between calculated inventories for the full-core and reflected plane model were small (on the order of 1-4\%). The differences between the two models scopes appeared to be the largest for the outermost radial zones (especially toward the inner reflector) and for the fissile plutonium isotopes ${ }^{239} \mathrm{Pu}$ and ${ }^{241} \mathrm{Pu}$. Each of these two nuclides shared a giant resonance at around $0.3 \mathrm{eV}$, which closely corresponded to the thermal peak of the neutron energy distribution within the core. Thus, the relatively higher thermal neutron flux within this area served to magnify small differences in the neutron energy spectrum in terms of the resulting weighted, one-group collapsed cross-section. Nonetheless, the relatively high degree of consistency between the two models appeared to justify using a reflected plane model as a reasonable simplification for purposes of tractability.

\subsection{COMPARISON OF HTGR AND LWR LIBRARY CHARACTERISTICS}

A useful reference point of comparison for the physics characteristics of the PBMR core is those of typical LWR assemblies. PWR and BWR assemblies admit substantial degrees of freedom in terms of design characteristics that influence the average neutron spectra (including but not limited to lattice design, initial enrichment, void fraction, soluble boron concentrations, and presence of control rods). The PBMR core nonetheless exhibited characteristics distinct from those of typical LWR assemblies, given the overall much higher level of moderation. The following sections provide a brief basis for comparison by illustrating the range of energies of the average lethargies of fission values calculated under broad sampling of the design space for BWR and PWR assemblies. Similar values were calculated for the fresh and equilibriumcomposition cores of the PBMR-400 and are presented in Table 7. All of the LWR data used for this comparison study are derived from calculations performed in [24].

\subsubsection{BWR ASSEMBLIES}

Figure 26 illustrates the distribution of EALF values for various BWR assembly designs, as calculated in [24]; for purposes of comparison, the range of EALF values for the PBMR system isotherms for the fresh and equilibrium-composition cores is also given. Notably, with the exception of cases evaluated at CZP conditions (i.e., $293 \mathrm{~K}$ fuel and moderator temperature and $0 \%$ void), the range of EALF values for each of the BWR lattices evaluated lies well above the range of values in each of the PBMR cases evaluated, with an average difference of over $+2.3 \mathrm{eV}$ for all cases evaluated and a median difference of about $+0.72 \mathrm{eV}$. Consistent with intuitive expectations, the average BWR EALF increases monotonically with void fraction (i.e., indicating a shift to a harder neutron spectrum driving fission), with an average difference of $+7.2 \mathrm{eV}$ from the maximum EALF of the PBMR cases at $90 \%$ void (and a median difference of $+4.0 \mathrm{eV}$ ), compared with an average difference of of $+0.31 \mathrm{eV}$ (median difference: $+0.21 \mathrm{eV}$ ) for the $0 \%$ void cases.

The GE14 assembly models evaluated in [24] specifically evaluate the effects of axial lattice zones (e.g., the dominant lattice zone (DOM), plenum region (PLE), vanished lattice zone (VAN) accounting for partiallength rods, and natural uranium axial blanket region (NAT)); this evaluation is broken out by lattice type in Figure 27. In general, the vanished lattice zone (VAN) zone and the power-shaping zone (PSZ) are strongly correlated with the hardest neutron spectra (and thus the larger differences in spectral behavior); but even regions associated with the dominant lattice show substantially higher EALF values than the range 


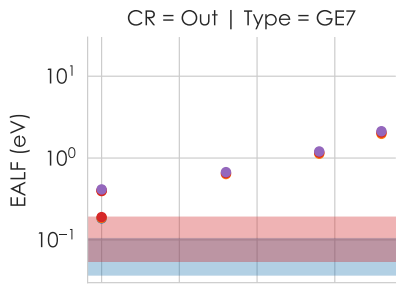

$\mathrm{CR}=\ln \mid$ Type $=\mathrm{GE7}$

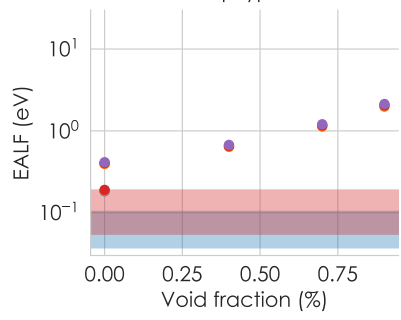

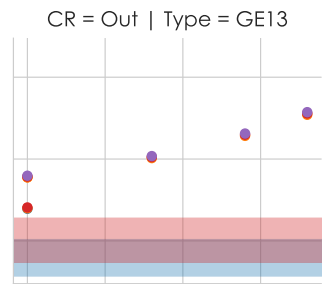

$\mathrm{CR}=\ln \mid$ Type $=\mathrm{GE} 13$

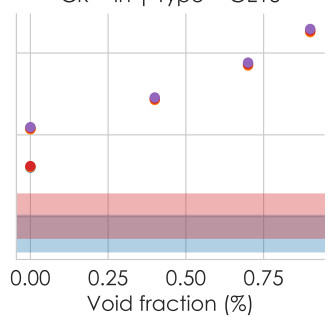

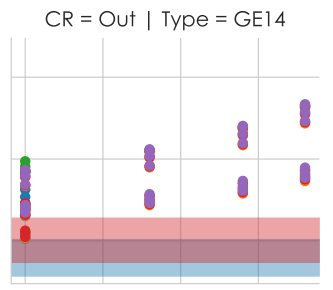

$\mathrm{CR}=\ln \mid$ Type $=\mathrm{GE} 14$

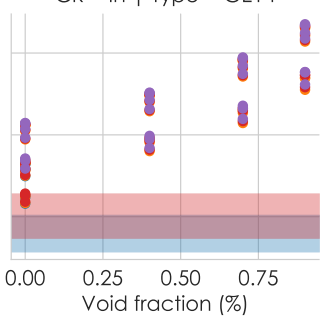

$C R=$ Out $\mid$ Type $=$ ATRIUM10

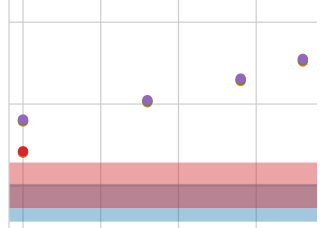

$\mathrm{CR}=\ln \mid$ Type $=$ ATRIUM10

中 293

ф 500

\$ 560

\$ 950

\$ 1500

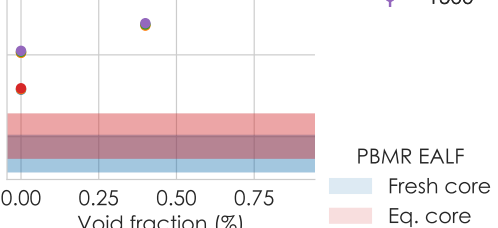

Figure 26. Energy of the average lethargy causing fission for various BWR assembly types under a range of moderator void fractions, fuel and moderator temperatures, and presence of control rods, with data from [24]. The calculated (overlapping) ranges of EALF values for the PBMR fresh and equilibrium-composition cores (calculated solely as a function of system isotherm temperature) are presented for comparison.
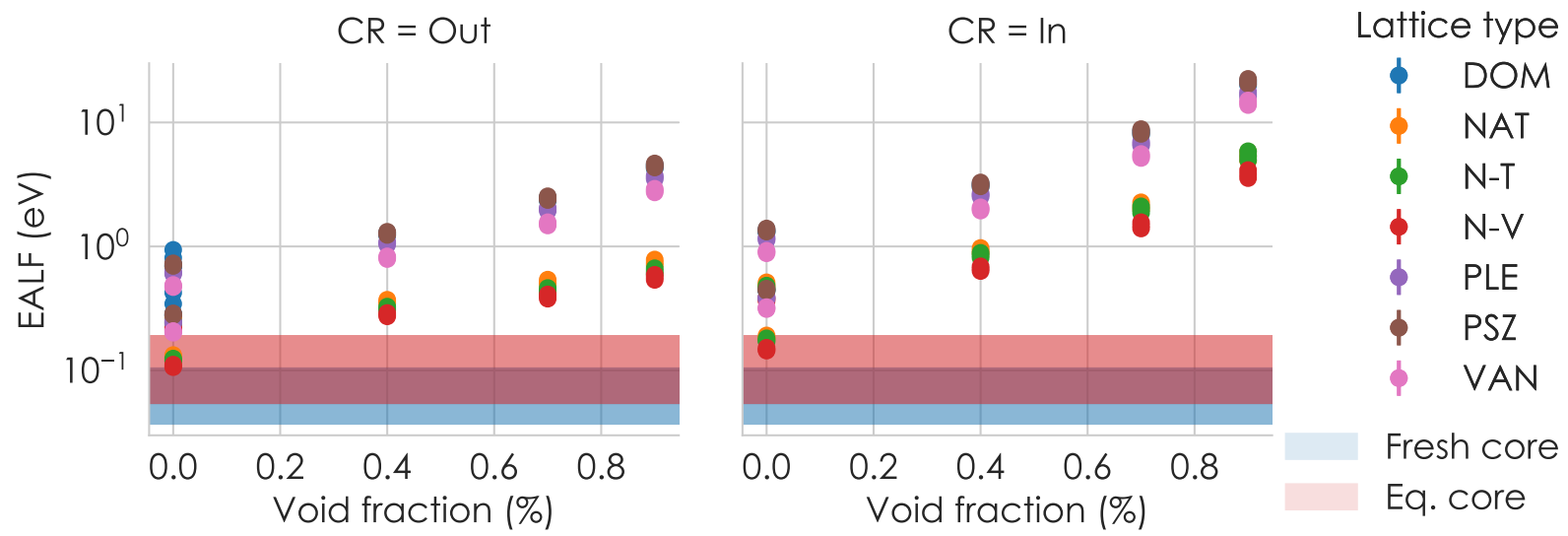

Figure 27. Energy of the average lethargy causing fission for a GE14 BWR assembly, broken out by lattice zone configurations per conventions defined in [25] (e.g., dominant lattice zone (DOM), powershaping zone (PSZ)), using data from [24]. The calculated (overlapping) ranges of EALF values for the PBMR fresh and equilibrium-composition cores (calculated solely as a function of system isotherm temperature) are presented for comparison. 
encompassed by the PBMR models. Thus the figure clearly illustrates the divergence in observed onegroup cross-section behavior for PBMRs (and thereby necessitates the development of new libraries and corresponding interpolation dimensions).

Figure 28 illustrates the collapsing spectra for the two most extreme cases of the GE14 BWR assembly in terms of the evaluated EALF values, with the CZP case representing the fuel and moderator at $293 \mathrm{~K}(0 \%$ void) and the hot full power (HFP) case representing the fuel at $500 \mathrm{~K}$ and the moderator at $560 \mathrm{~K}(90 \%$ void). Both cases are presented for the dominant lattice.

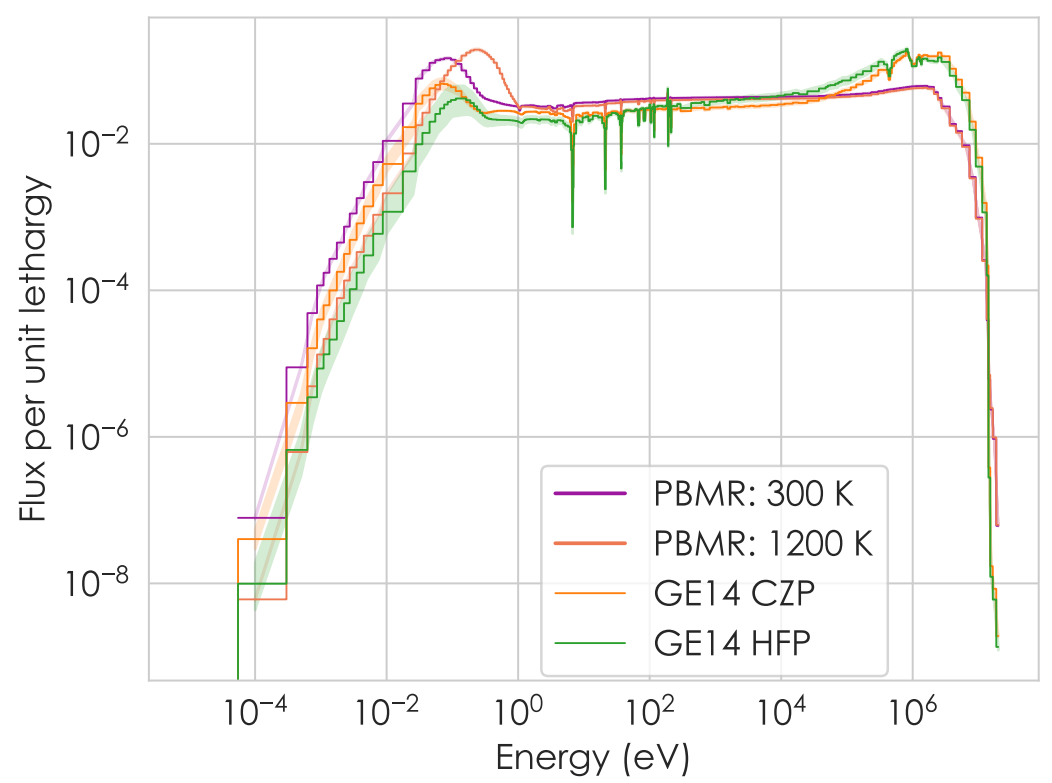

Figure 28. Comparative lethargy-weighted multi-group energy spectra for the GE14 fresh assembly under CZP (0\% void) and HFP (90\% void) (representing relative lethargy extrema for the BWR cases considered), alongside the PBMR equilibrium-composition cores at 300 and $1200 \mathrm{~K}$. Shaded regions represent variations over different (non-Gd) fuel regions within the assembly.

In Figure 28 the more thermalized nature of the PBMR core (combined with the relatively high temperature sensitivity) is evident. Notably, it can be seen that despite the rather large shift in the thermal peak at the highest temperature isotherm for the PBMR, the overall flux balance is far more weighted toward the thermal peak relative to the fast (fission) peak, thereby indicating a much softer spectrum than the range of typical BWR assemblies. 


\subsubsection{PWR ASSEMBLIES}

Similar to the BWR assemblies studied, Figure 29 presents collected EALF data for PWR assemblies over dimensions of lattice design, initial enrichment, moderator and fuel temperature, presence of control rods, and soluble boron concentration. Differences in the system EALF are typically smaller than those in the more extreme regions of the BWR cases (i.e., high-void regions); however the differences in spectral hardness are still significant, with an average difference in EALF of $+0.75 \mathrm{eV}$ (and a median difference of +0.4 $\mathrm{eV})$.
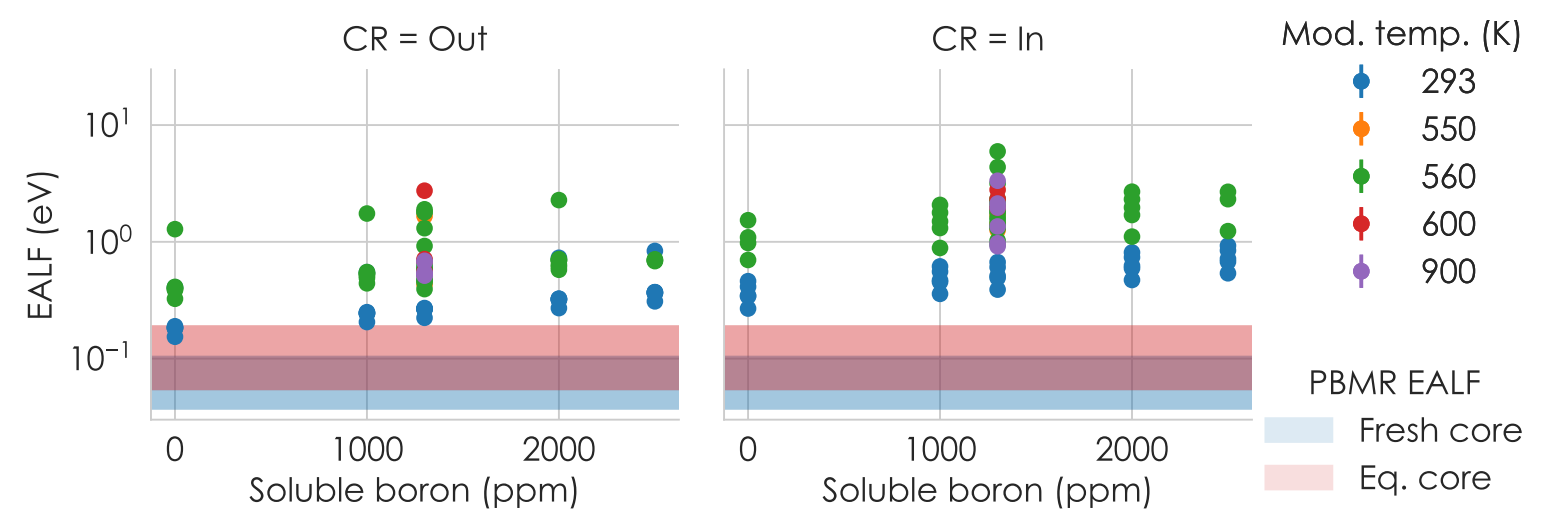

Figure 29. Energy of the average lethargy causing fission for various PWR assembly types under a range of initial fuel enrichments, fuel and moderator temperatures, soluble boron concentrations, and presence of control rods, with data from [24]. The calculated ranges of EALF values for the PBMR fresh and equilibrium-composition cores (calculated solely as a function of system isotherm temperature) are presented for comparison.

Within the PWR data, there was a substantially lower degree of variance in EALF values in the evaluated permutations, chiefly owing to the general lack of substantial changes in the average moderator density (with a standard deviation of $0.83 \mathrm{eV}$ in the EALF of all samples considered). While the average difference from the PBMR cores was smaller, it was nonetheless large enough to mark a shift of the thermal neutron flux well into the region of the ${ }^{240} \mathrm{Pu}$ giant resonance (thereby producing the strong burnup-dependent effect on the corresponding one-group removal cross-section not observed in the PBMR data). This finding is most evident in referring back to Figures 22 and 23. 


\section{CONCLUSIONS}

In this report, we present an analysis of HTGR modeling capabilities in SCALE, using the PBMR-400 benchmark as a test case. The scope of this analysis includes a review of existing SCALE capabilities for handling doubly-heterogeneous fuel (such as the TRISO fuel pebbles used in the PBMR-400), a brief review of prior HTGR systems analysis work performed using SCALE, and an in-depth analysis of the neutronic characteristics of the PBMR-400 core at startup and equilibrium conditions so as to evaluate the necessary prerequisite information for producing ORIGEN reactor data libraries for rapid depletion analyses.

In terms of the neutronic characteristics of the PBMR-400 core, the annular core geometry with graphite moderators produces a relatively flat radial flux profile, so that the overall difference in flux across a given radial zone within the pebble-loaded core region is relatively flat for a given axial location. However, the circulating nature of the fuel (i.e., fuel is loaded from the top of the core and transits axially downward and is cycled through the core multiple times), produces a sharp axial flux and power gradient at equilibrium conditions. The power strongly tilts toward the top of the core (where the reactivity is highest). However, the overall spectral characteristics, such as the ratio of the thermal to fast neutron flux, remain relatively constant through the axial length of the core. Moderate differences in this ratio (the "spectral index") were observed in the radial regions closest to the graphite reflectors, potentially warranting the consideration of radial position for depletion library development. However, given the relatively invariant flux shape axially within a given radial zone, this result would imply that specific axial treatment of the pebbles is not required for properly capturing depletion behavior.

With respect to the general form of the neutron energy spectrum, the lethargy-weighted spectrum shape in both the fresh and equilibrium-composition cores showed a strongly bifurcated shape, with a prominent thermal-region peak and a proportionally smaller fast (fission neutron) peak with a small, upward-trending gradient in the lethargy-weighted flux between the thermal and fast peaks. Given the considerably softer spectrum (i.e., thermally weighted) compared with traditional LWR spectra, this shape resulted in noticeably higher response sensitivity to parameters such as the system temperature. The system temperature had a first-order impact on both depletion characteristics and in terms of reactor reactivity coefficients and EALF. Notably, in the PBMR-400 system, system temperature (treated as a system isotherm) had the effect of shifting the thermal peak higher in energy, producing a narrower peak with the median energy shifted much closer to the range of 0.1-0.5 eV. This results in a shift of both the peak and centroid of the thermal neutron distribution closer to the ${ }^{239} \mathrm{Pu}$ absorption giant resonance located around $0.3 \mathrm{eV}$. The expected result of this shift was twofold: parasitic capture by ${ }^{239} \mathrm{Pu}$ resulted in a strong negative reactivity feedback (observed as the temperature reactivity coefficient) and in significantly enhanced production of transuranic species (influencing depletion inventories). As a result, system temperature effects must be considered for depletion calculations for HTGR systems like the PBMR-400.

The implications of the observed physical behavior of the PBMR-400 system for ORIGEN library development are discussed in the following section.

From a system modeling perspective, although SCALE's present capabilities for physics treatment of doubly-I heterogeneous fuel are mature and well-developed, the analysis of the PBMR-400 core indicated specific feature gaps necessary to perform full-fidelity analysis of the system physics behavior. These features include treatment of the continuously circulated pebbles as well as the distribution of pebbles throughout the core. These feature gaps, along with the associated remediating assumptions, will be discussed in the following sections. 


\subsection{DEPLETION MODELING FROM A “PEBBLE-LEVEL”APPROACH}

One of the overarching assumptions employed and demonstrated through the course of this analysis has been the treatment of depletion from effectively the level of an individual pebble - that is, considering the problem of evaluating depletion behavior by considering the spectral characteristics of the core as seen from the level of the individual fuel pebble as it makes multiple transits through the core. In this framework, the depletion behavior of the pebble thus primarily depended on factors such as its radial location relative to the reflector regions and reactor-level characteristics such as the graphite reflector temperature. This approach is in contrast to treating the depletion behavior of a given pebble as being contingent upon the state of its surrounding neighbor pebbles.

In effect this approach assumed a relatively loose coupling among individual pebbles within the core. That approach was borne out by evaluating the neutronic characteristics (i.e., spectrum shape) for reduced-order models such as the "reflected plane" pebble model compared with those of the full core. In effect, for a given pebble as it transits through the core, for the equilibrium cycle condition, the variation in the neighboring pebbles between any given cycle is expected to be small and largely contingent upon the pebble's radial location within the core. Because pebbles are continuously circulated through the system (with pebbles being recirculated until they reach their terminal burnup and fresh pebbles are continuously added), the adjacent fuel throughout the core at any given time is effectively static with respect to axial position (e.g., as a layer of fuel transits downward through the core, new fuel is added back to the top)

Thus, the treatment of the individual pebble as the unit of depletion (and the focus of one-group library development) is not unlike the approach presently employed for LWR assemblies, wherein the granular level of interest is that of the assembly (i.e., ORIGEN data libraries are developed assuming an infinitely reflected assembly). In the case of generating LWR reactor libraries, it is generally assumed that the assembly's location within the core is of negligible consequence, save for characteristics such as control blades / control rods, and so on; i.e., prior experience in radiochemical validation studies performed using ORIGEN generally confirm that this approach is sufficient to give accurate results.

For the PBMR system, the neutron moderation is strongly impacted by the annular graphite reflector regions. Meanwhile, the factors that influenced the relative neutron flux shape-namely, absorption by other pebbles-were likewise governed by the relative radial position with respect to the reflectors. Hence, in treating the system across one dimension (i.e., radial position), this situation accounted for endogenous effects like competing thermal neutron absorption by neighboring fuel pebbles.

With this perspective in mind, it is reasonable to approach depletion calculations for systems like this one by examining the reactor environment from the "pebble" perspective, i.e. primarily in terms of pebble's relative radial location during its history and its initial design characteristics (e.g., initial enrichment, TRISO particle packing). This method has the effect of greatly simplifying the approach to calculating isotopic inventories for the core under equilibrium conditions.

With this in mind, this "pebble supercell" approach would require further investigation regarding its validity under the conditions leading up to the equilibrium-composition core, particularly as graphite dummy pebbles are continuously replaced for new fuel pebbles (thereby slowly drawing down the graphite pebble inventory and increasing the fuel pebble inventory). In this scenario, the axial variation of material within the core is no longer effectively static but rather is continuously evolving as burned fuel is returned to the top of the annular region alongside fresh pebbles. While it is likely that the spectral variations would continue to be dominated by the proximity to the radial reflector regions, such an assumption would need to be further vetted before being fully applied to studying the depletion behavior of a nonequilibrium starting core condition. 


\subsection{RECOMMENDATIONS FOR ORIGEN LIBRARY DEVELOPMENT}

One of the chief aims of this study was to characterize the parameters needed to develop ORIGEN reactor data libraries that provide sufficient coverage of the problem domain to be useful for rapid depletion calculations (i.e., by interpolating pre-generated libraries to problem-dependent conditions). From the analysis of the PBMR-400 benchmark problem, three general dimensions stand out as most significant for library development: the pebble initial enrichment, burnup, and graphite temperature. Notably, separate design parameters such as the TRISO geometry and particle packing fraction were not investigated in the scope of this study. They would be expected to likewise require differential library development.

The observed evolution of one-group cross-sections with initial enrichment and burnup was not unexpected and is in fact consistent with previous experience with LWR fuel modeling. However, the graphite reflector/moderator temperature also exhibited a profound impact upon the neutron spectrum and thus the calculated one-group cross-sections. Specifically, changes in the graphite temperature drive changes in neutron scattering rates, thereby resulting in a narrowing and shifting of the thermal neutron peak into regions that are closer to prominent actinide capture resonances. As a result, system temperature was identified as a new dimension for library interpolation and development that has heretofore not been considered in the development of ORIGEN libraries for LWRs and similar systems.

\subsection{RECOMMENDED DEVELOPMENT PRIORITIES FOR ORIGAMI}

The most significant new capabilities required for ORIGAMI to model HTGR systems like the PBMR400 fall roughly into two categories: library processing and specification of time-dependent power history information.

\subsubsection{ENHANCEMENTS TO LIBRARY INTERPOLATION}

With respect to library processing, ORIGAMI currently relies on internal calls to the legacy module ARP (Automatic Rapid Processing), which performs interpolation of select uranium oxide (UOX)-based fuel cross-sections based on predefined assembly-level characteristics (including initial enrichment, moderator void fraction, and mid-cycle burnup). While ARP is also capable of interpolating mixed oxide (MOX)based libraries based on initial characteristics such as the plutonium vector and fraction of total plutonium, as of SCALE 6.2 ORIGAMI only supports UOX-based fuel types. Although adapting the ARP module to handle characteristics of TRISO-based fuel is possible, a more maintainable long-term approach would be to leverage previously developed capabilities for linear interpolation across an arbitrary number of dimensions using on-library descriptor information [26]. This capability was developed in support of anticipated future needs for more generalized ORIGEN library interpolation capabilities for scenarios such as this one; however it has yet to be deployed as a user-facing feature, partly owing to the need to update upstream code infrastructure (i.e., the TRITON sequence) to provide information required for interpolation directly on the library file itself. As ORIGAMI begins to support libraries beyond typical UOX-based LWR assemblies, the need for this infrastructure development becomes more apparent.

Within the context of adapting ORIGAMI for PBMR depletion applications, the logical set of interpolation dimensions for ORIGEN libraries would thus span a 4D space, consisting of radial location within the core, initial pebble enrichment, system temperature (more precisely, graphite reflector temperature), and pebble burnup. While the effect of burnup is relatively subdued for cases such as the equilibrium-composition core, the effect is substantially more pronounced for the fresh core. Interpolation along the burnup dimension is handled automatically by ORIGAMI; likewise, at present, ORIGAMI automatically infers initial uranium enrichment using the material composition. A more general approach may involve providing more specific input to the user for overlaying characteristic dimensions of interest to the problem, similar to the behavior of 
the axial and radial power map features (used to infer mid-cycle burnups by modifying the nominal specific power specified in the irradiation history block).

For example, an alternative means of capturing arbitrary interpolation dimensions would be to have the user specify a series of "maps" covering the depletion zones within the problem; each map would specify a characteristic dimension capable of being interpolated along a real-value axis (e.g., initial fuel enrichment, radial location relative to the reflectors, fuel and/or reflector temperature). Each "interpolation map" would functionally exist parallel to others, with the problem-specific features of each depletion "zone" determined through the permutation of the layered map entries. For example, consider the three sets of parallel maps in Figure 30 overlaid upon one another to form a set of nine unique tuples, specifying the initial enrichment, radial location, and start-of-cycle burnup. This would then result in a set of nine unique permutations, shown as Table 11.

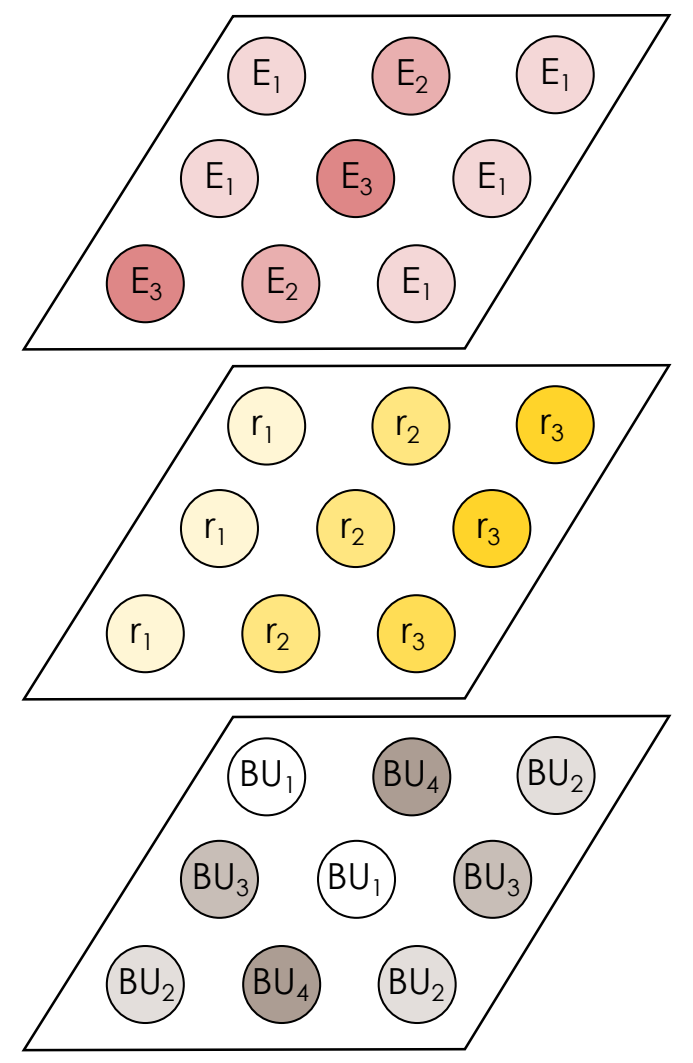

Figure 30. Proposed series of "map overlays" for interpolation of libraries with arbitrary dimensions in ORIGAMI; the combination of the maps produces a unique tuple for each depletion location ("pebble"), resulting in the permutations shown in Table 11.

Table 11. Pebble "permutations" of initial enrichment, radial location, and starting burnup, as given by the overlaid tables shown in Figure 30.

\begin{tabular}{lll}
\hline$\left(E_{1}, r_{1}, B U_{1}\right)$ & $\left(E_{2}, r_{2}, B U_{4}\right)$ & $\left(E_{1}, r_{3}, B U_{2}\right)$ \\
$\left(E_{1}, r_{1}, B U_{3}\right)$ & $\left(E_{3}, r_{2}, B U_{1}\right)$ & $\left(E_{1}, r_{3}, B U_{3}\right)$ \\
$\left(E_{3}, r_{1}, B U_{2}\right)$ & $\left(E_{2}, r_{2}, B U_{4}\right)$ & $\left(E_{1}, r_{3}, B U_{2}\right)$ \\
\hline
\end{tabular}




\subsubsection{SPECIFYING FLOWING-PEBBLE DEPLETION HISTORIES}

Accommodating depletion history information for flowing pebbles requires some re-conceptualization of the depletion model used in ORIGAMI to obtain fuel inventories. In its original formulation, ORIGAMI presumes a static assembly consisting of discrete axial and radial "zones" subject to a common nominal power history overlaid with axial and radial power gradient information. Here, the fuel in each depletion zone is always subject to one gradient-based "modifier" to the power history information. (This is in addition to other local features which may modify the spectrum, such as the axial void profile).

By contrast, the flowing pebble scenario involves transit through an axial gradient over its residency in the core; hence, what was once a static modifier of a nominal power history now becomes the time-dependent power factor acting on a global core power. Moreover, in the context of MEDUL cycles, each separate pass through the core involves movement of the pebble into a separate spectral zone.

Further complicating matters is the inherently stochastic and branching nature of pebble depletion histories. In the absence of a prescribed core reloading pattern, pebbles are effectively assumed to be distributed randomly between radial zones as they are reloaded at the top of the core. Because a given pebble can transit through one of multiple radial (spectral) zones with each pass, the possible irradiation histories leading up to a given pebble history necessarily branches with each pass, with the accumulation of the isotopic inventory (particularly for the transuranic species which are dependent upon multiple neutron captures) dependent upon not only the present trajectory but the passes before it.

As such, the inventory of a pebble at a particular location and pass number through the core is no longer a single, deterministic value but rather represents a distribution of values encompassing each possible series of paths through the core. Accounting for this can be performed in one of two ways:

1. At the end of an irradiation pass through the core, determine the discharge inventory for the pebble given the history within each radial / spectral zone. Following this, produce a "normalized" end-ofcycle inventory via a probability-weighted sum of each of the discharge inventories, i.e.:

$$
\overline{N^{\prime}}(t)=\sum_{i=1}^{N_{\text {zones }}} N_{i}^{\prime}(t) P_{i}
$$

where $P_{i}$ is the probability of a pebble being loaded into zone $i$. The homogenized inventory is then used as the starting inventory for the parallel depletion history calculations for the next cycle. Thus, the depletion inventory of a single pebble after $k$ passes through the core consisting of $j$ zones is simply $(k-1) \cdot j+1$ (i.e., given that the location of pebble in the "last" pass for which inventories are being calculated is known).

2. At the end of each irradiation pass through the core, the discharge compositions for each zone are used to seed a new branching calculation for each zone in the next depletion cycle. i.e., rather than rehomogenizing the concentrations to produce a "weighted" prior-batch concentration, the end-of-cycle inventory for each cycle is then used as the starting point for calculations over $j$ zones. Hence, the inventory of the pebble at cycle $k$ is the superposition of all possible states for $k-1$ cycles, and hence the number of calculations required is:

$$
N_{\text {calculations }}=j^{k-1}+1
$$

i.e., for each cycle $k$, the pebble can be in one of $j$ states. The end result of this calculation is thus a histogram distribution of inventories for each isotope, the variance of which will depend on its respective sensitivity to the incident neutron spectrum. 
For comparison, assuming a core composed of five radial zones, calculating the pebble inventory at its sixth and final pass would lead to $(6-1) \cdot 5+1=26$ separate calculations for the "homogenized" case and $5^{5}+1=3126$ calculations for the "branching" treatment. While both are reasonably tractable given the easily parallelized structure of the problem, they illustrate the complexity of determining inventories for the discharge inventory for even a single pebble in the core.

While an initial assumption would be that differences from prior cycles would effectively "wash out" (thus favoring the homogenized approach to pebble depletion), prior work by Gougar et al. indicate that the variance in plutonium inventories (i.e., ${ }^{239} \mathrm{Pu}$ and ${ }^{240} \mathrm{Pu}$ ) can be non-trivial [22]. Simulating the trajectories of pebbles across 4 radial zones over 10 passes (yielding $4^{10}=1048576$ separate depletion histories), they found significant variation in discharge inventories of ${ }^{239} \mathrm{Pu}$ (as high as $\pm 23 \%$ over a relative uniform distribution), indicating a high sensitivity to the irradiation history [22]. This occurred despite the fact that the pebble discharge burnup was normally distributed around $81 \frac{\mathrm{GWd}}{\mathrm{MTIHM}}$ with an observed range of 76.6-87.8 $\frac{\text { GWd }}{\text { MTIHM }}$ [22]. Masses for other notable species such as ${ }^{240} \mathrm{Pu}$ as well as fission products of interest such as ${ }^{134} \mathrm{Cs}$ and ${ }^{109} \mathrm{Ag}$ generally exhibited distributions closer to a Gaussian distribution (with ${ }^{240} \mathrm{Pu}$ showing a moderate skew-right behavior indicative of the influence of the preceding ${ }^{239} \mathrm{Pu}$ distribution) [22].

From a user interface perspective, regardless of the implementation choice taken for tracking inventories between cycles (i.e., homogenization or branching), a conceptual approach would be to allow the user to define factors such as the bins of an axial power shape, pebble residency time within axial locations, and the probability distribution of pebbles between radial zones, wherein ORIGAMI would account for pebble shuffling throughout the history. An example implementation is illustrated as Figure 31.

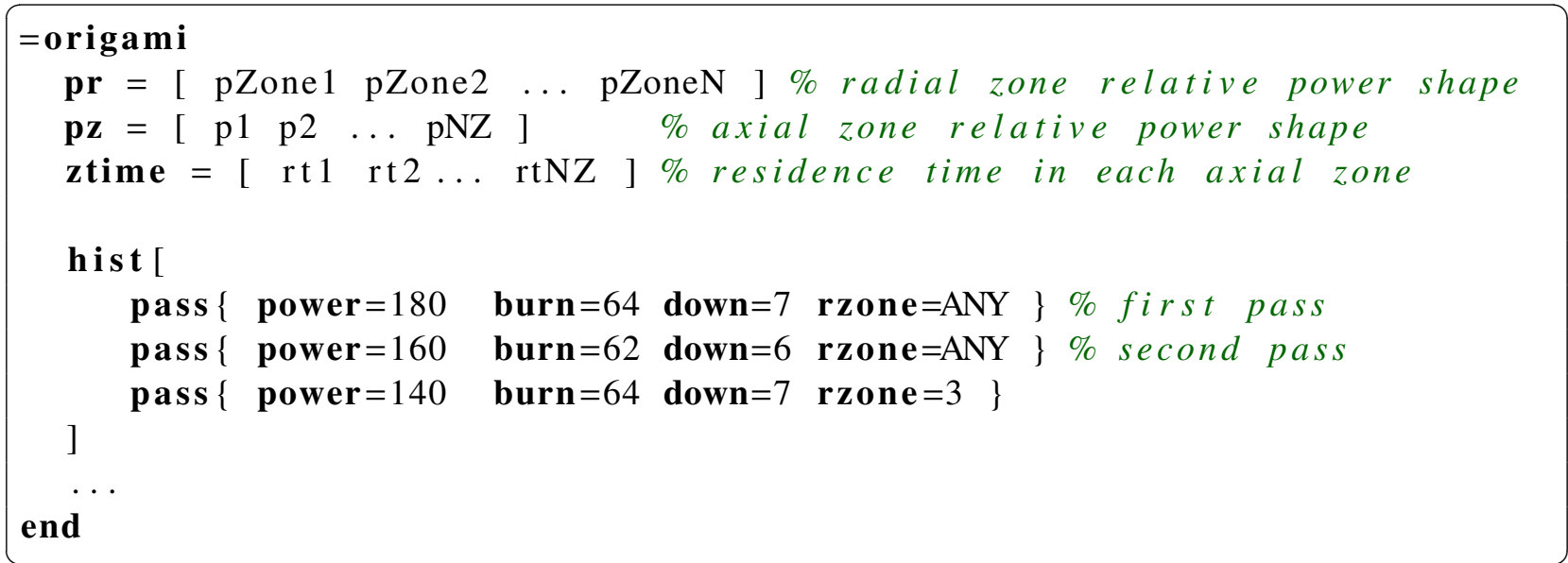

Figure 31. Sample implementation of an ORIGAMI interface for flowing-pebble HTGR systems.

In the conceptual example within Figure 31, pebbles are assumed to exist in any radial zone for the first and second passes and are in the third radial zone for the third pass. Meanwhile, the nominal core power varies for each pass, modified by the radial power shape (pr keyword) and axial power profile ( $\mathrm{pz}$ profile modifier). Other options would include relative residence time within axial zones (ztime) and user-defined probability distributions between radial zones.

\subsection{ADDITIONAL ANALYTICAL CAPABILITIES TO BE DEVELOPED}

The continuous circulation of fuel pebbles within the PBMR system introduces unique challenges for neutron transport, including capturing the effects of the evolving neighboring fuel pebbles on the depletion spectrum for a given pebble of interest. Because the shape of the neutron spectrum appears to be relatively 
constant axially, explicit tracking does not appear to be required to accurately capture depletion behavior over a single pass through the core. However, because pebbles are continuously placed back into the core, the distribution of previously burned pebbles radially is expected to produce impacts on overall depletion behavior (in terms of both the individual pebble tracked and the influence of neighboring pebbles). This work has not sought to analyze this specific effect at this time; however plans are to evaluate this specific effect in future follow-on work.

The assumption of the core at equilibrium alleviates some of this challenge in that it is assumed that the core composition generally reaches steady-state with respect to the axial dimension; thus, pebble transit within a cycle can effectively be captured by examining different axial locations. However, this approach assumes prior information about the equilibrium-composition core composition and does not easily lead to methods for determining an equilibrium composition from a given starting core configuration. Thus, while the analysis of the equilibrium-composition core within this study can generally be regarded as valid (insofar as the benchmark results themselves represent a valid representation of the equilibrium system), a means of tracking time-dependent pebble positions during multiple transit cycles is necessary for establishing the intermediate, transient core compositions and for calculating equilibrium conditions based upon alternative initial conditions (e.g., core length, burnup per cycle, initial enrichment). Note that similar systems (such as the Berkeley Mk. 1 FHR) present an identical modeling challenge, and thus the issue of continuously shuffled fuel goes beyond the HTGR class of systems alone.

Additionally, one challenge noted in the development of the PBMR-400 model is the pebble packing and placement itself. Although the use of a dodecahedral lattice afforded placement of pebbles at the correct packing density (61\%), this approach had notable issues, namely with respect to local packing voids located at the annular boundary when partial pebbles ("clipped" pebbles) were not allowed. This issue produced substantial deviation in the system $\mathrm{k}_{\text {eff }}$ compared with the problem benchmark. Although modeling the system with clipped pebbles at the core boundary produced better agreement with the benchmark, this approach is suboptimal both because it does not convey a physically realistic portrayal of the system and because it still produces higher than anticipated divergence from the benchmark.

A preferable solution would be to implement a RCP array geometry capability, preferably with a userconfigurable packing fraction. Experience with other transport codes (such as SERPENT) has indicated that modeling the system using an RCP geometry produces better agreement overall with the reference benchmark without relying on non-physical compromises such as pebble truncation at the reactor boundary [27]. Presently, although SCALE has introduced random particle placement capabilities for TRISO fuel particles, that approach does not represent a tractable method for full-core modeling. Rather, it is suitable for evaluating the effects of TRISO particle distribution within an individual pebble. Notably however, the implementation of particle tracking using random-unit geometries is a non-trivial exercise; SERPENT handles this via Woodcock delta tracking; other codes such as RMC have implemented using random universe geometry methods [28]. 


\section{REFERENCES}

[1] International Atomic Energy Agency (IAEA), Status and Prospects for Gas Cooled Reactor Fuels, IAEA-TECDOC-CD-1614. Vienna, Austria: IAEA, Apr. 2009, IsBN: 978-92-0-152809-4.

[2] - Evaluation of High Temperature Gas Cooled Reactor Performance: Benchmark Analysis Related to the PBMR-400, PBMM, GT-MHR, HTR-10 and the ASTRA Critical Facility, IAEA-TECDOC-I 1694. Vienna, Austria: IAEA, Apr. 2013, ISBN: 978-92-0-137610-7.

[3] Nuclear Science Committee, Nuclear Energy Agency (NEA), PBMR Coupled Neutronics / Thermalhydraulics Transient Benchmark The PBMR-400 Core Design, NEA/NSC/DOC(2013)10. Paris, France:I OECD/NEA, Jul. 2013, vol. 1: The Benchmark Definition.

[4] “SCALE Code System," in, W. A. Wieselquist, R. A. Lefebvre, and M. A. Jessee, Eds., 6.2.4, ORNL/TM-I 2005/39. Oak Ridge, TN: Oak Ridge National Laboratory, 2020.

[5] G. Ilas, D. Ilas, R. P. Kelly, and E. E. Sunny, "Validation of SCALE for High Temperature Gas-Cooled Reactor Analysis," Oak Ridge National Laboratory, Oak Ridge, TN, Tech. Rep. NUREG/CR-7107 (ORNL/TM-2011/161), Jul. 2012.

[6] E. Sunny and G. Ilas, "SCALE 6 analysis of the HTR-10 pebble-bed reactor for initial critical configuration," in PHYSOR 2010: Advances in Reactor Physics to Power the Nuclear Renaissance, Pittsburgh, PA, May 2010.

[7] J. D. Bess and T. Ivanova, Eds., International Handbook of Evaluated Reactor Physics Benchmark Experiments (IRPhEP), NEA No. 7496. Paris, France: Nuclear Energy Agency / Organisation for Economic Co-operation and Development (OECD/NEA), Jul. 2019. [Online]. Available: https : //www . oecd-nea.org/download/science/wprs/irphep-handbook/2019/.

[8] S. Goluoglu, L. M. Petrie Jr., M. E. Dunn, D. F. Holenbach, and B. T. Rearden, "Monte Carlo criticality methods and analysis capabilities in SCALE," Nuclear Technology, vol. 174, no. 2, pp. 214-235, 2011. Dor: 10.13182/NT10-124. [Online]. Available: https://doi .org/10.13182/NT10-124.

[9] T. M. Pandya, S. R. Johnson, T. M. Evans, G. G. Davidson, S. P. Hamilton, and A. T. Godfrey, "Implementation, capabilities, and benchmarking of Shift, a massively parallel Monte Carlo radiation transport code," Journal of Computational Physics, vol. 308, pp. 239-272, 2016, Issn: 0021-9991. Dor: $10.1016 /$ j. jcp. 2015.12.037. [Online]. Available: http: //www. sciencedirect. com/ science/article/pii/S0021999115008566.

[10] M. L. Williams, "Resonance self-shielding methodologies in SCALE 6," Nuclear Technology, vol. 174, no. 2, pp. 149-168, 2011. Dor: 10.13182/NT09-104. [Online]. Available: https: //doi .org/10. 13182/NT09-104.

[11] S. Choi, C. Kong, D. Lee, and M. L. Williams, "A New Equivalence Theory Method for Treating Doubly Heterogeneous Fuel-II: Verifications," Nuclear Science and Engineering, vol. 180, no. 1, pp. 41-57, 2015. Dor: 10.13182/NSE14-72. [Online]. Available: https: //doi . org/10.13182/ NSE14-72.

[12] M. L. Williams, S. Choi, and D. Lee, "A New Equivalence Theory Method for Treating Doubly Heterogeneous Fuel-I: Theory," Nuclear Science and Engineering, vol. 180, no. 1, pp. 30-40, 2015. DoI: 10.13182/NSE14-68. [Online]. Available: https://doi .org/10.13182/NSE14-68.

[13] G. Ilas, "On SCALE validation for PBR analysis," in PHYSOR 2010: Advances in Reactor Physics to Power the Nuclear Renaissance, Pittsburgh, PA, May 2010.

[14] F. Bostelmann, C. Celik, M. L. Williams, R. J. Ellis, G. Ilas, and W. A. Wieselquist, "SCALE capabilities for high temperature gas-cooled reactor analysis," Annals of Nuclear Energy, vol. 147, Nov. 2020. Dor: $10.1016 / \mathrm{j}$. anucene. 2020.107673. 
[15] H. Rütten, K. Haas, H. Brockmann, and W. Scherer, "V.S.O.P. (99/05) Computer Code System," Institut für Sicherheitsforschung und Reaktortechnik, Forschungszentrum Jülich, Jülich, Germany, Tech. Rep. Jül-4189, Oct. 2005.

[16] W. Wieselquist, S. Hart, A. Isotalo, F. Havlůj, S. Skutnik, R. Lefebvre, I. Gauld, D. Wiarda, J. Lefebvre, G. Hu, N. Sly, and D. Lago, "Scale code system, version 6.2," in, ORNL/TM-2005/39. Oak Ridge, TN, USA: Oak Ridge National Laboratory, Apr. 2020, ch. 5.1 ORIGEN: Neutron Activation, Actinide Transmutation, Fission Product Generation, And Radiation Source Term Calculation, pp. 5-21-5-24.

[17] H. Rütten, “The Depletion Computer Code ORIGEN-JÜEL-II,” Forschungszentrum Jülich, Jülich, Germany, Tech. Rep. Jül-2739, Mar. 1993.

[18] D. She, J. Guo, Z. Liu, and L. Shi, "Development of a neutronics analysis code for pebble-bed htrs," Nuclear Engineering and Design, vol. 329, pp. 192-197, 2018, Issn: 0029-5493. Dor: 10.1016/j . nucengdes.2017.09.018. [Online]. Available: https://www. sciencedirect. com/science/ article/pii/S0029549317304594.

[19] H. D. Gougar, F. Reitsma, and W. Joubert, "A comparison of pebble mixing and depletion algorithms used in pebble-bed reactor equilibrium cycle simulation," in International Conference on Mathematics, Computational Methods E Reactor Physics (ME C 2009), Saratoga Springs, NY, May 2009.

[20] H. Gougar, W. Yoon, and A. Ougouag, "Multiscale Analysis of Pebble Bed Reactors," in HTR 2010, INL/CON-10-19081, Oct. 2010.

[21] H. D. Gougar, "The Application of the PEBBED Code Suite to the PBMR-400 Coupled Code Benchmark - FY 2006 Annual Report," Idaho National Laboratory, Idaho Falls, ID, Tech. Rep. INL/EXT06-11842, Sep. 2006.

[22] H. D. Gougar, A. M. Ougouag, W. K. Terry, and K. N. Ivanov, "Automated Design and Optimization of Pebble-Bed Reactor Cores," Nuclear Science and Engineering, vol. 165, no. 3, pp. 245-269, Dor: 10.13182/NSEQ8-89.

[23] C. Stoker, F. Reitsma, and Z. Karriem, "Creation of the Equilibrium Core PBMR Origen-S Cross Section Library," in Proceedings of HTR-2002, Petten, The Netherlands, Apr. 2002.

[24] U. Mertyurek, M. A. Jessee, and B. R. Betzler, "Lattice physics calculations using the embedded selfshielding method in polaris, Part II: Benchmark assessment," Annals of Nuclear Energy, vol. 150, p. 107 829, Jan. 2021, IssN: 0306-4549. Dor: 10.1016/j . anucene.2020.107829. [Online]. Available: http: //www. sciencedirect.com/science/article/pii/S0306454920305272.

[25] M. L. Fensin, "Optimum Boiling Water Reactor Fuel Design Strategies to Enhance Reactor Shutdown by the Standby Liquid Control System," Master's thesis, University of Florida, 2004.

[26] N. Sly, S. Skutnik, and W. Wieselquist, "Portable reactor data library interpolation via self-describing ORIGEN libraries," in Proceedings of PHYSOR 2016, Sun Valley, ID, May 2016, pp. 2218-2231.

[27] V. Rintala, H. Suikkanen, J. Leppänen, and R. Kyrki-Rajamäki, "Modeling of realistic pebble bed reactor geometries using the Serpent Monte Carlo code," Annals of Nuclear Energy, vol. 77, pp. 223230, 2015, Issn: 0306-4549. Dor: 10.1016/j . anucene. 2014.11.018. [Online]. Available: https: //www. sciencedirect.com/science/article/pii/S0306454914006008.

[28] S. Liu, Z. Li, K. Wang, Q. Cheng, and D. She, "Random geometry capability in RMC code for explicit analysis of polytype particle/pebble and applications to HTR-10 benchmark," Annals of Nuclear Energy, vol. 111, pp. 41-49, 2018, IssN: 0306-4549. Dor: 10.1016/ j . anucene . 2017. 08. 063. [Online]. Available: https: / / www. sciencedirect.com/science/article/pii / S0306454917302724.

[29] mvw(https://math.stackexchange.com/users/86776/mvw), Hexagon packing in a circle, Mathematics Stack Exchange, URL:https://math.stackexchange.com/q/1283124 (version: 2015-05-19). [Online]. Available: https://math.stackexchange.com/q/1283124. 
APPENDIX A. DEVELOPMENT OF A COMPACT SPHERICAL PACKING ALGORITHM 


\section{APPENDIX A. DEVELOPMENT OF A COMPACT SPHERICAL PACKING ALGORITHM}

A key constraint to developing a realistic PBR geometry is the compact placement of pebbles within the cylindrical or annular space. For hard spheres, HCP represents the highest theoretical packing efficiency (around 67\%); this, however, is a higher packing efficiency than the problem benchmark (which specifies a packing fraction of about $61 \%$ ). Meanwhile, correctly maintaining the fuel-to-moderator ratio demands a minimum of pebble geometry clipping at the core edges (reinforcing the hard spheres assumption).

To address these issues while allowing for efficient generation of very large pebble arrays, we developed a sphere packing algorithm to calculate pebble-filled units of a triangular-pitched hexagonal lattice, derived from [29]. This section will describe the algorithm used to obtain efficient sphere packing within the hexagonal lattice while attempting to minimize clipping of sphere boundaries to maximally preserve the fuel-to-graphite ratio within the core.

The problem of determining hexagonal unit cells that lie "inside" the outer annular boundary but "outside" the inner boundary can be solved by determining the locations of each unit cell's vertices. For the outer boundary, if the radial displacement of the vertex from the center of the annular region exceeds the outer radius, the vertex is thus "outside" (meaning that any unit sphere would be clipped); thus the cell is left empty. Similarly, if any vertex displacement vector is smaller than the inner radius, the hexagonal unit can likewise be declared "outside" (and thus remain empty).

The calculation of vertex locations then simply requires the formulation of a displacement vector to describe the location of each cell's vertices relative to a reference cell (in this case, the central cell). This formulation can be represented as two vector components representing two dimensions of travel (vertical and horizontal displacement), referred to as $u_{0}$ and $u_{1}$. This displacement vector $\vec{u}=\left(u_{0}, u_{1}\right)$, defined as Eq. (A.1) [29].

$$
\begin{aligned}
u_{0} & =2 d \cdot\left(\hat{i}+0.5\left[\begin{array}{ll}
\hat{i} & \bmod 2
\end{array}\right]\right) \\
u_{1} & =2 \cdot \frac{\sqrt{3}}{2} d \cdot \hat{j} \\
& =\sqrt{3} d \cdot \hat{j}
\end{aligned}
$$

Here, $\hat{i}$ and $\hat{j}$ represent an integer number of units displaced from the central unit, and $d$ corresponds to the peak-to-flat dimension of the hexagonal unit cell. (It is assumed that the sphere radius $r$ is equal to $d$, resulting in close-packing on the plane.) Therefore, the apothem (distance from the center of the hexagon to any vertex) is simply $a=\frac{\sqrt{3}}{2}$. Likewise, one should note the modulo term for $u_{0}$, which accounts for the fact that the lattice centers shift to the right by a half-unit for every other row (as a result of the close-packed arrangement).

Thus if $D$ is defined as the outer circle diameter of a circle that bounds the vertices of a hexagon (i.e., each vertex lies on the circle), it is apparent that $D=2 a=\sqrt{3} \cdot d$, where $d$ is the center-to-flat dimension. Thus, the location of vertex $k$ for a hexagon displaced as $\vec{u}=\left(u_{0}, u_{1}\right)$ is given as Eq. (A.2) [29].

$$
v_{k}=\left(D \cdot \cos \left(\frac{(2 k+1) \pi}{6}\right), D \cdot \sin \left(\frac{(2 k+1) \pi}{6}\right)\right) \text { where } k \in[0 . .5]
$$

Finally, we can establish the boundary conditions for a vertex to be considered "inside" the annular region as Eq. (A.3) [29]. This condition reduces to the norm of the vertex coordinate $\left(v_{k}\right)$ and the displacement vector 
$(\vec{u})$, which can then be compared directly against the inner and outer annular radii ( $R_{i}$ and $R_{o}$, respectively). Note that this boundary condition is effectively a combination of two cylindrical boundary conditions (i.e., "outside" the inner circle forming the annular boundary and "inside" the outer circle).

$$
R_{i} \leq\left\|v_{k}+\vec{u}\right\| \leq R_{o}
$$

To ensure the correct heavy metal mass in the core, a small tolerance factor $\varepsilon$ is introduced in order to relax the boundary condition, thereby allowing cells to be filled that would result in trivial clipping of the fuel pebble graphite layer. 
APPENDIX B. NEUTRONIC ANALYSIS OF THE FRESH CORE 


\section{APPENDIX B. NEUTRONIC ANALYSIS OF THE FRESH CORE}
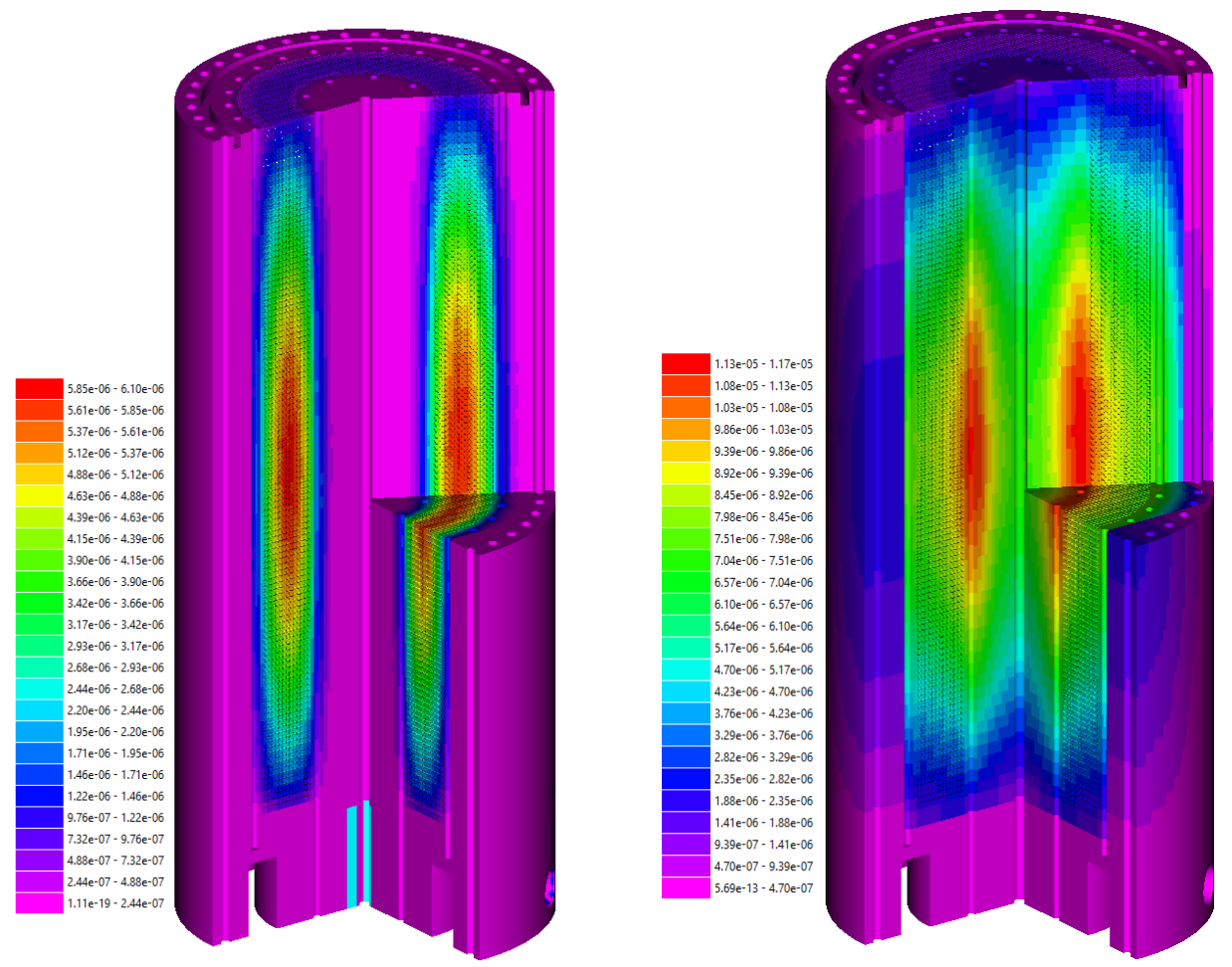

Figure 32. PBMR-400 flux distribution for the fresh (initial) core; (left) fast neutron group ( $E>1.86$ eV), (right) thermal group $(E \leq 1.86 \mathrm{eV})$

Figure 33 illustrates the spectral index for the fresh core. Compared to the equilibrium-composition core (Figure 10), the overall flux profile of the fresh core is substantially more thermalized, with overall less distinction between radial zones. Thus, while the outer radial zones still show greater thermalization than the interior zones, this effect is substantially diminished compared to the equilibrium-composition core. 

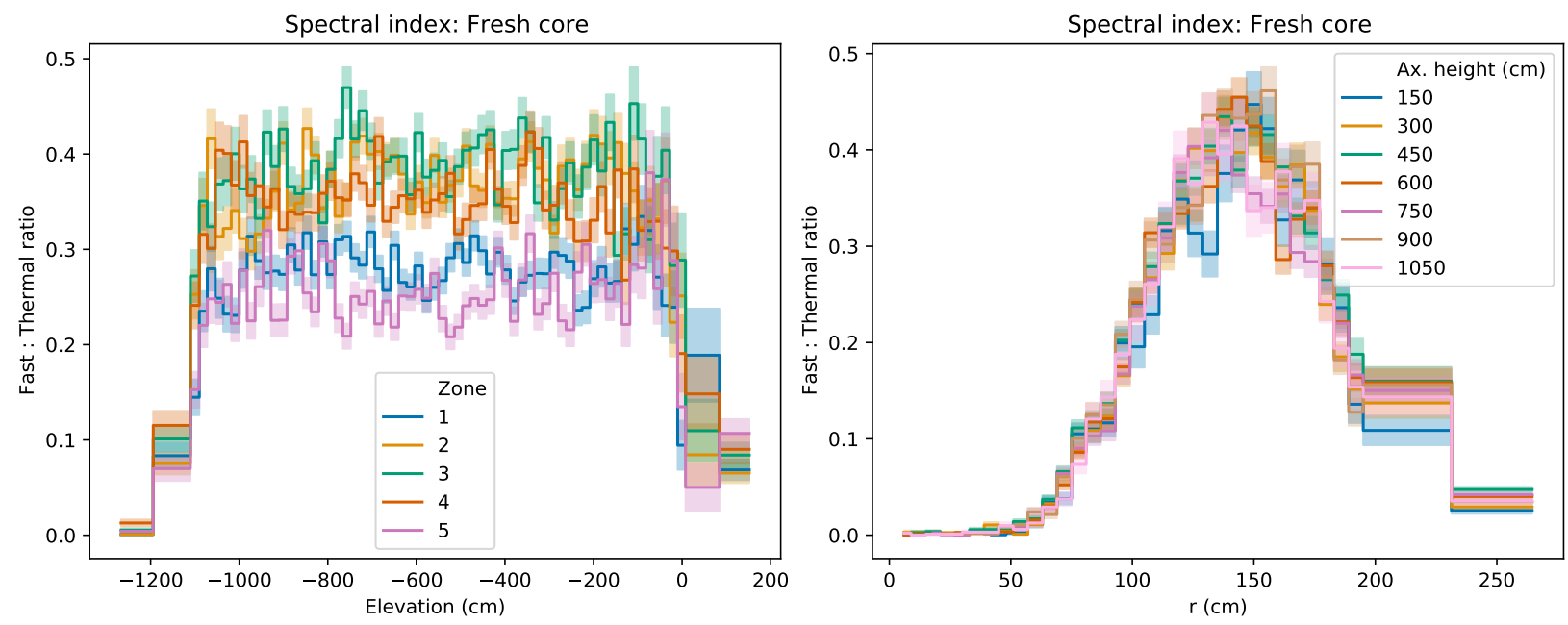

Figure 33. Spectral ratio (fast : thermal flux) for the fresh core, along the (left) axial dimension, (right) radial dimension; the thermal-fast boundary is defined at $E=1.86 \mathrm{eV}$. 